UCRL-LR-130400

\title{
An Investigation into the Accuracy, Stability and Parallel Performance of a Highly Stable Explicit Technique for Stiff Reaction-Transport PDEs
}

\author{
Al Franz
}

February 17, 1998

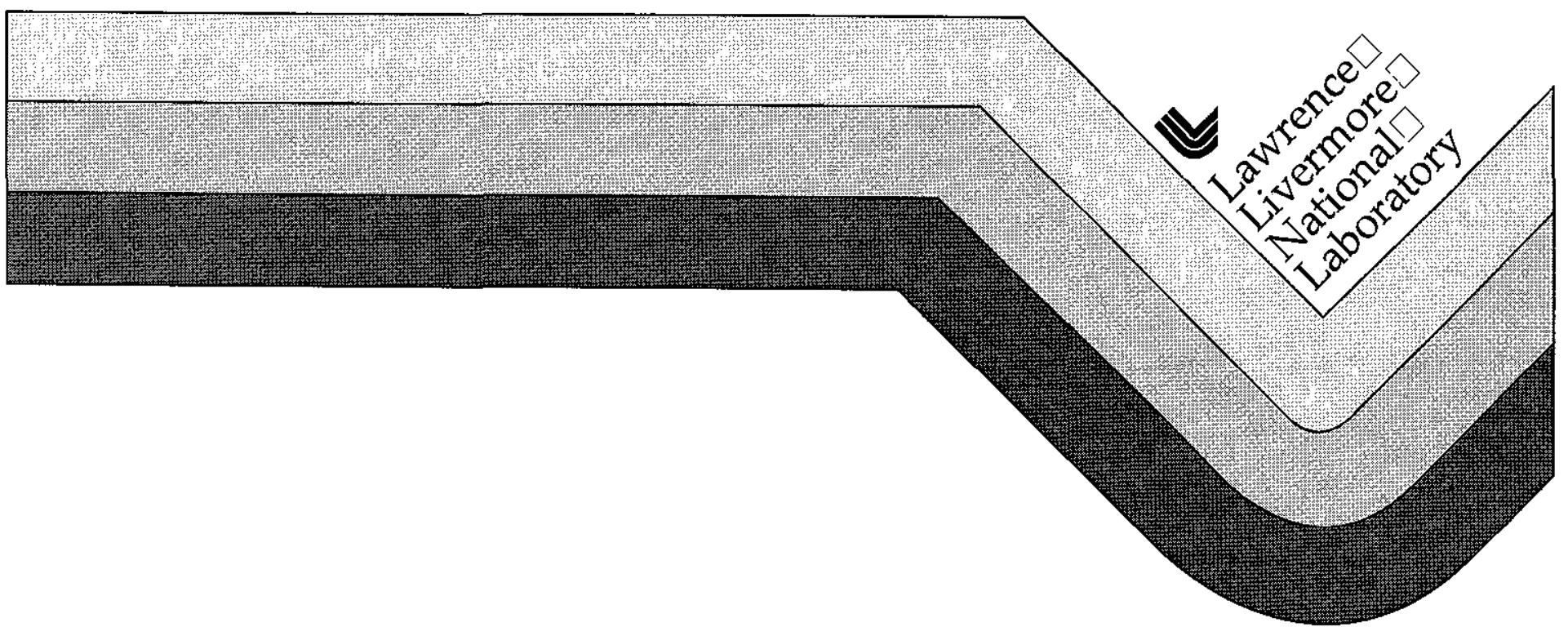




\section{DISCLAIMER}

This document was prepared as an account of work sponsored by an agency of the United States Government Neither the United States Government nor the University of California nor any of their employees, makes any warranty, express or implied, or assumes any legal liability or responsibility for the accuracy, completeness, or usefulness of any information, apparatus, product, or process disclosed, or represents that its use would not infringe privately owned rights Reference herein to any specific commercial product, process, or setvice by trade name, trademark, manufacturer, or otherwise, does not necessarily constitute or imply its endorsement, recommendation, or favoring by the United States Government or the University of California The views and opinions of authors expressed herein do not necessarily state or reflect those of the United States Government or the University of California, and shall not be used for advertising or product endorsement purposes

This report has been reproduced directly from the best available copy

Available to DOE and DOE contractors from the

Office of Scientific and Technical Information

PO Box 62, Oak Ridge, TN 37831

Prices available from (423) 576-8401

Available to the public from the National Technical Information Service

US Department of Commerce 5285 Port Royal Rd,

Springfield, VA 22161

Work performed under the auspices of the US Department of Energy by Lawrence Livermore National Laboratory under Contract W-7405-ENG-48 


\title{
An Investigation into the Accuracy, Stability and Parallel Performance of a Highly Stable Explicit Technique for Stiff Reaction-Transport PDEs
}

\author{
Al Franz \\ Master of Science \\ Thesis
}

Manuscript date: February 17, 1998 



\title{
An Investigation into the Accuracy, Stability and Parallel Performance of A Highly Stable Explicit Technique for Stıff Reaction-Transport PDEs
}

\author{
A Thesis Presented to the Graduate Faculty \\ of
}

California State Iniversity, Hayward

\author{
In Partial Fulfillment \\ of the Requirements for the Degree \\ Master of Science m Computer Science
}

By

Al Fianz

February 17,1998 
AN INVESTIGATION INTO THE ACCURACY, STABLLITY AND PARALLEL PERFORMANCE OF A HIGHLY STABLE EXPLICIT TECHNIQUE FOR STIFF REACTION-TRANSPORT PDES

By

Al Franz

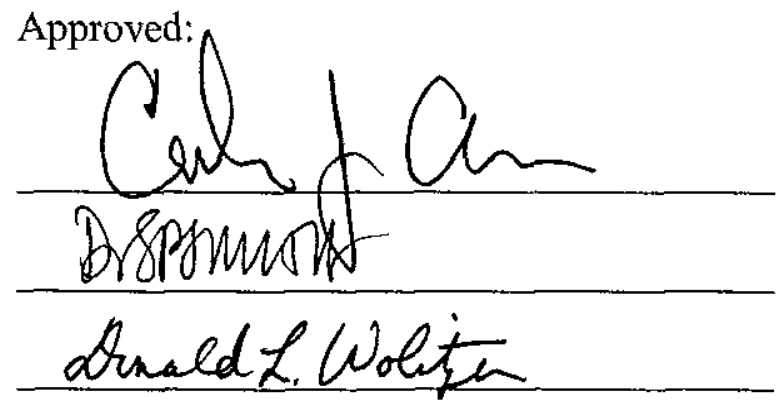

Date:

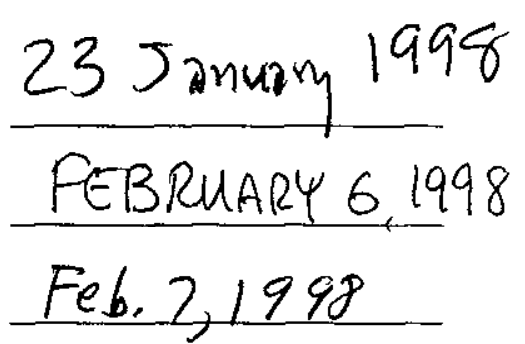





\section{Abstract}

The numerical simulation of chemically reacting flows is a topic that has attracted a great deal of cuisent research At the heart of numerical reactive flow simulations are large sets of coupled, nonlinear Partial Differential Equations (PDEs) Due to the stiffness that is usually present, explicit time differencing schemes are not used despite their inherent simplicity and efficiency on parallel and vector machines, since these schemes require prohibitively small numerical stepsizes Implicit time differencing schemes, although possessing good stability characteristics, introduce a great deal of computational overhead necessary to solve the simultaneous algebraic system at each timestep This thesis examines an algorithm based on a preconditioned time differencing scheme The algorithm is explicit and permits a large stable time step An investigation of the algorithm's accuracy, stability and performance on a parallel architecture is presented 


\section{Acknowledgments}

I would like to acknowledge the strong support of Colin Aro, whose technical knowledge, experience and suggestions were vital to the success of this project I would also like to thank Don Wolitzer and Garry Rodrigue for their pioneering work in the field of Preconditioned Time Differencing, Stuart P Smith for his extensive mathematical knowledge, Dale Slone for his work on the parallel performance study, Alan Hindmarsch for his intricate knowledge of stiff differential systems, Ted Billard for expert $\mathrm{L}_{\mathrm{E}} \mathrm{X}$ typesetting advice, Charlie Harper for his conceptual approach to physical problem solving, and Daryl W P1eston and Louis Villanueva for their priceless suggestions on how to conduct labor atory iesearch, all of whom played a role, wittingly or otherwise, in this endeavor 


\section{Contents}

1 Introduction 1

2 History of Related Research 5

3 Preconditioned Time Differencing $\quad 8$

4 The Algorithm 10

5 The Test Problem 12

6 Investigation of the Method 13

61 Introduction 13

62 Stability : 13

63 Accuiacy 14

64 Parallel Peiformance 15

65 Speed 16

66 Output 18

7 Conclusion $\quad 21$

8 Appendices 22

A Physics Background 23

A 1 Traveling Waves $\quad$ : 23

A 2 Simple Harmonic Motion 25

A 3 Damped Hasmonic Motion 26

A 4 Thermodynamics 26

A 5 The Navier-Stokes Equations 27

A 6 The Advection-Diffusion Equation $\quad 27$

A 61 Pure Convection 28

A 62 Pure Diffusion $\quad 29$

A 63 'The Heat Equation $\quad 29$

A 7 Light 29

B Chemistry Backgı ound 30

B 1 Rates of Reactions 30

B 2 Photodissociation 33

B 3 The Chapman Mechanism 34

C Mathematics Background $\quad 36$

C 1 Power Series $\quad 36$

C 11 Taylon's Series $\quad 36$

C 12 Maclaurin Sei ies $\quad 36$

(1 13 Taylor's Theorem with Remainder $\quad 36$

C 2 linear Systems 37

C. 21 Matrices 38

C 22 Eigenvalue Problem 40

('3 Lipschitz Condition 40

C. 4 Differential Equations $\quad 40$

C. 41 Ordinary Differential Equations 41

C 42 Systems of Ordinary Differential Equations 43

C 43 Partial Differential Equations 44 
D Numerical Methods $\quad 47$

D 1 Intioduction $\quad 47$

D 2 Euler's Method 48

D 3 Explicit Methods 49

D 4 Inplicit Methods $\quad 50$

D 5 One-Step Method 50

D 6 Local Truncation Erior

D 7 Total Accumulated Error 51

D 8 Order of Accuiacy 51

D 9 Stability $\quad 52$

D 10 Stiffness

D 11 Functional Iteration

D 12 Newton-Raphson Method $\quad 55$

D 13 The Trapezoidal Rule $\quad 56$

D 14 Linear Multistep Methods

D 15 Piedictor-Cor rector Methods 56

D) 16 Adaptive Stepsize $\quad 57$

D 17 Runge-Kutta Methods $\quad 57$

D 18 Iter ative Techriques for Linear Systems 58

D 181 Jacobi Iteration $\quad 59$

D 182 Gauss-Seidel Iteration 60

D 183 Relaxation Method 61

D 184 Matrix-Splitting 61

D 19 Subroutine Packages $\quad 62$

E Parallel Processing $\quad 63$

F Related Methods $\quad 66$

F 1 Introduction 66

F 2 Finite Difference Approximations to Derivatives 66

F 3 Finite Difference Method $\quad$ ' 66

F 4 Finite Element Method 67

F 5 Numerical Method of Lines 68

F 6 Method of Characteristics

F 7 Particle Methods 70

F 8 Monte Callo Method 70

G Related Problems 72

G 1 The Non-linear Burgers' Equation $\quad 72$

G 2 The Fisher Equation 72

$\begin{array}{ll}\text { H Notation } & 73\end{array}$

$\begin{array}{ll}\text { I Abbreviations } & 77\end{array}$ 


\section{Introduction}

Atmospheric chemistry deals with chemical compounds in the atmosphere, their distribution, onigin, chemical transformation into other compounds, and finally, their removal from the atmospheric domain These substances may occur as gases, liquids, or solids The composition of the atmosphere is dominated by the gases nitrogen and oxygen in pioportions that have been found invariable in time and space at altitudes up to $100 \mathrm{~km}$ All other components are minor ones with many of them occurring only in traces Atmospheric chemistry thus deals primarily with trace substances

As an interdisciplinary field of science, atmospheric chemistry has its main roots in meteorology and chemistry, with additional ties to miclobiology, plant physiology, oceanography, and geology The full range of the subject was last treated by (; E Junge in his 1963 monograph "Ait Chemistry and Radioactivity" The extraordinarily rapid development of the field in the past two decades has added much new knowledge and insight into atmospheric processes Most of the literature, however, addresses specifically the problems of local air pollution, whereas the natural atmosphere has received only a fragmentary treatrnent even though it provides the yardstick for any assessment of air pollution levels The recognition that man has started to pertuub the atmosphere on a global scale is now shifting the attention away fiom local toward global conditions [1]

In the labolatory, in the chemical manufacturing industry, and in many other situations, chemical reactions occur under relatively uniform conditions of temperature, pressure, and irradiation In the atmosphere these conditions vary substantially, producing noticeable effects on composition and chemical reactions Therefore, it is wor thwhile to present some information on the gaseous medium within which this chemistıy occurs

The atmosphere is densest at the earth's surface The density decieases sapidly with increasing altitude Since many chemical reactions are pressure-dependent, the density structure alone implies differences in composition Most reactions are temperature-dependent as well and thus reflect to some extent the atmospheric temperature structure

Reactions involving solar photons are central to atmospheic chemistry One therefore needs to know the spectrum of solar radiation in some detail This spectrum is approximately that of a blackbody at $5900^{\circ} \mathrm{K}$, crossed by mumerous absorption lines Several gases modify the solar spectrum as the radiation penetiates further into the atmosphere $\mathrm{O}_{3}, \mathrm{O}_{2}, \mathrm{H}_{2} \mathrm{O}$, and $\mathrm{C}^{\prime} \mathrm{O}_{2}$ are the most important modifiess, but contributions are also made by $\mathrm{CH}_{4}, \mathrm{~N}_{2} \mathrm{O}$, and other natural and anthropogenic gases [2] The absorption of light, piimarily by $O_{3}$, is a major factor causing the increase in temperature with altitude in the stratosphere [3]

Of special importance to atmospheric chemistry are the photons energetic enough to dissociate photosensitive molecules, for most molecules such photons have wavelengths shorter than about $400 \mathrm{~nm}$ The solar fluxes increase significantly as one moves upward from the giound toward the tıopopause (the troposphere-stıatosphere boundary) Similarly diamatic variations are seen at constant altitudes as one moves to different latitudes or as the seasons change Any reactions dependent upon solar photons obviously vary in proportion to these changes

Another important facet of atmospher ic chemistıy not always present in other chemical systems is atmospheric mixing On a small scale, these motions are turbulent, and serve to disperse emissions from point sources On a largel scale, the motions are ordered and have the ability to transport emitted species far downwind and to high altitudes The large scale mixing is not uniform over the earth Transport along a fixed latitude band is surh that emittants can circumnavigate the earth in about two weeks, as shown by volcanic eruption clouds In the lower troposphere, typical advection velocities are of order $25 \mathrm{~km} / \mathrm{h}$, but valiations due to local weather patterns are substantial Within a single hemisphere, northsouth transport occurs over a time scale of a few weeks Transport across the equator is strongly inhibited by the convergence of opposed air flows, and requires six months to a yeal 
Vertical mixing is a strong function of local weather patterns also A simplified conceptual approach often taken to describe this mixing is to assume that the flux of a species in a chosen direction is proportional to its mean gradient This "eddy flux," due to the random motions of the atmosphere, is given by

$$
\phi=-K \frac{d C}{d z}
$$

where $C$ is the concentration, $z$ is the altitude, and $K$ is the eddy diffusion coefficient The eddy coefficient is approximately constant throughout the troposphere, indicating relatively uniform mixing The eddy coefficients at and above the tropopause are quite low, then. increase as mixing again becomes reasonably efficient in the middle and upper stratosphere

'The residence times of atmospheric trace species are closely related to the mixing reflected by these eddy coefficients Some months are required to vertically mix species emitted at. the ground throughout the tropospinere Transport across the tropopause is quite slow, since. mixing is inhibited by the atmosphenic temperature structure Several years are required for an average chemically inert molecule to move from the ground to the lower stratosphere

More than $999 \%$ of the molecules comprising the earth's atmosphere are nitiogen $\left(N_{2}\right)$, oxygen $\left(\mathrm{O}_{2}\right)$, or one of the rare gases In addition, many trace molerules are found in the atmosphere They are capable of influencing or controlling celtain atmospheric processes, even though their total concentration is very low Carbon dioxide, which is an important factor in the earth's sadiation balance but is chemically unreactive in the troposphere, has an average (but increasing) concentration of about 330 parts per million (ppm) The most abundant of the reactive gases is methane, which comprises less than two parts per million of the tropospheric gas Other reactive species are still less prevalent, the combined concentration of all of the reactive trace gases in the atmosphere seldom totals $10 \mathrm{ppm}$

A most important molecule for atmospheric chemistry, especially droplet chemistry, is water vapor The mixing 1 atio of water vapor in the troposphere varies by some five orders of magnitude, from a few parts per hundred in the tropies near the surface to less than one part per thousand over the poles at the surface and to a few parts per million near the tropopause $\Lambda$ s a consequence of the mixing of the atmosphere, instantaneous water concentrations at a given altitude and location vary by about a factor of ten depending on the instantaneous vertical motion patterns

The atmosphere is also influenced by acrosol particles, clouds and precipitation [2]

Interpretations of atmospheric behavior can be tested by companing the predictions of a model of the atmosphere with results from measurements on the atmosphere itself Diagnostic models are used to assess hypotheses about the physics and chemistry of atmospheres from a knowledge of present-day physical and chemical structure Prognostic models are of interest in evaluating the future behavior and evolution of an atmosphere subject, for example, to changes in natural and artificial inputs of trace species Such models can also be used to determine whether an assumed set of "starting" conditions could lead to observed present-day atmospheres

Consider a volume element in an atmosphere small enough to be uniform with respect to all variables such as temperature, density, composition, and so on If the rate of flux to and fiom that element of heat, radiation, matter, etc, from all other atmospheric elements is calculated, then the rate of change of the various physical parameters can be established Chernical change within the volume element requires only slight modification for the local alterations in composition, and possibly in physical conditions The continuity equations involved in the models are typified by the one for matter, which states that the net flow of mass into unit volume per unit time is equal to the local rate of change of density Solution of the continuity equations tor every physical and chemical parameter of interest, and for every volume element in the atmosphere, should then lead to a self-consistent model of atmospheric behavion that mimics in all respects the temporal and spatial changes in the real atmosphere According to this view, with sufficient input information, and a fine enough 
grid size for the volume elements, all meteorological as well as chemical phenomena could be simulated by the model Such three-dimensional models (3-D) would be ideal for studying atmospheric chemistry Computer-numerical solutions are naturally used in models, but the 3-D models are so demanding of computer time and memory that, at present, the chemistry has to be simplified drastically [4] Simpler chemical models may then be more practical, e g the Chapman model of ozone distribution

The numerical treatment of reaction-transport problems has attracted a great deal of current interest Application areas such as combustion [5], groundwater flow [6], climate modeling [7], atmospheric science [8], and chemical oceanography [9] all iely heavily on the numerical solution of reaction-transport PDEs

The modeling of atmospheric chemistry involves an equation with a chemical kinetics part and an advective-diflusive part The chemical kinetics part is

$$
\frac{d_{i} u}{d t}={ }_{i} R(\mathbf{u}, t)={ }_{i} P(\mathbf{u}, t)-{ }_{i} \hat{L}(\mathbf{u}, t)_{i} u-{ }_{i} \bar{L}(\mathbf{u}, t)_{i} u^{2},
$$

where $\mathbf{u}=\left({ }_{1} u, \quad, N u\right)^{T}$ represents a vector of chemical concentrations, with ${ }_{i} u={ }_{i} u(t)$ being the concentration of the $i$ th chemical species and $N$ being the total number of species involved Equation (2) models the molecular reactions that change chemical species into other chemical species The source term ${ }_{i} R$ describes the reactions that occur between chemical species This ieaction operator is generally nonlinear and couples together the reaction equations for all species involved ${ }_{i} P(\mathbf{u}, t),_{i} \hat{L}(\mathbf{u}, t)$, and ${ }_{i} \bar{L}(\mathbf{u}, t)$ are the production and loss rates Important to note is that the production and loss rates, while functions of $\mathbf{u}$, do not depend upon the component ${ }_{i} u[10]$ (a reaction rate depends upon the concentrations of the reactants not the concentrations of the products [11]) Furthermore, a reaction rate at a point does not depend upon the spatial distribution of reactants, $i$ e the concentiation of reactants at other spatial points 'Thus, equation (2) describes a system of Ordnary Differential Equations (ODEs)

The advective-diffusive part

$$
u_{t}+a u_{x}=D u_{x x},
$$

where $u=u(x, t)$, models the transport (translational movement, eddies, intermingling) of the species

When combined, these equations take the general form

$$
\frac{\partial\left({ }_{i} u\right)}{\partial t}+a \quad \nabla\left({ }_{i} u\right)=D \nabla \quad \nabla\left({ }_{i} u\right)+{ }_{i} R(\mathbf{u}, t), \quad i=1, \quad, N
$$

where $\mathbf{u}=\left({ }_{1} u, \quad,{ }_{N} u\right)^{I}$ represents a vector of chemical concentrations, with ${ }_{i} u={ }_{i} u(x, t)$ being the concentration of the $i$ th chemical species Equation (4) describes the basic conservation laws for each species (no atoms are created or destroyed in this simulation, which describes nothing lower than molecular reactions)

With the emexgence of large-scale parallel platforms, full 3-D reactive flow simulations have begun to appear [12] These 3-D simulations can consume enol mous amounts of piocessor time [13], which (an severely limit the length of the simulation that can reasonably be car ried out Thus, current research strives to develop increasingly efficient algorithms for numerically solving these reaction-transport PDE systems

Explicit time-differencing schemes have the advantage that they ate simple to implement and very efficient on parallel and vector computers The disadvantage in using explicit schemes is the serious constraint placed upon the size of their stable timestep intervals By contıast, implicit time-stepping schemes are often not constıained by numerical stability, but 1equire a great deal noore effort to implement An implicit time-stepping srheme ralls for the solution of a potentially nonlinear system of simultaneous algebraic equations at ear $h$ timestep This adds a great deal of overhead to the calculation and can offset the gain in efficiency due to the increase in stepsize made possible by the latger stable timestep inter val 
The reaction mechanism in the reaction-transport PDE system can lead to the presence of widely disparate time scales in the modeling scenario This situation gives rise to the phenomenon of stiffness [14], whereby the constraint on the size of explicit stable timestep intervals is exacer bated by limiting the numerical stepsize to the smallest timescale in the simulation In this case, the use of an implicit numerical time-stepping scheme is necessary [15] Thus, the considerable computational overhead brought about by the implicit scheme is primarily the result of the Ieaction operator ${ }_{i} R \quad[13]$

This thesis addresses a numerical method for simulating the atmospheric chemistry described by equation (4) The audience is expected to possess basic knowledge equivalent to a bachelor's degree in computer science Knowledge of some disciplines of science is expected of such a person, eg calculus, linear algebra, the essentials of physics and chemistry, and the basic notions of computer science Some principles of mathematics, physics, chemistry and parallel processing, howeves, occur as fundamental to the understanding of the physical problem, mathernatical model, numerical method, sjecific algorithm and experimental results addressed by this thesis Therefore, crucial ideas with which the reader may not be farniliar are discussed in the appendices as background material An attempt was made to order these sections so that any concept which depends upon others will be preceded by those prerequisite concepts However, as this may not always be convenient, some ideas in this text. may depend upon material to be defined thereafter

The notation $u$ will represent a vector $\left(u_{1}, \quad, u_{q}\right)^{T}$ of spatial points $\mathbf{u}$ will represent a vector $\left({ }_{1} u, \quad, N u\right)^{T}$ of chemical concentrations as described previously Variables with hats $(\grave{y}, \hat{f}$, etc) will usually denote vectors unless indicated to the contrary The chenical production and loss rates $\left({ }_{i} P(\mathbf{u}, t),{ }_{i} \hat{L}(\mathbf{u}, t)\right.$, and $\left.{ }_{i} \bar{L}(\mathbf{u}, t)\right)$ are scalar functions unless described differently 'The test, problem, which was used to verify the algorithm being investigated, is a simple 1-D reaction-transport problem which demonstrates many of the important difficulties seen in larger systems Unless noted otherwise, we shall assume that, as in the test problem, the spatial discretization of $u$ is in one dimension only (longitude, $x$, in units of ievolution), along the earth's equator, with $0 \mathrm{rev} \leq x<1 \mathrm{rev}$, although in general, $\hat{u}$ is discretized in latitude, longitude and altitude, as well as time Similarly, we shall assume that $\mathbf{u}=\mathbf{u}(x, t)$ has only one spatial dimension, when in general, $\mathbf{u}$ is a function of latitude, longitude and altitude, as well as time Time, $t$, will normally be in units of seconds (s) 


\section{History of Related Research}

The chemically reactive advection-diffusion equation has its origins in the simple advectiondiffusion equation

The pure advection equation takes the form

$$
u_{t}+a u_{x}=0, \quad 0 \leq x \leq L, \quad 0 \leq t,
$$

while the pule diffusion equation takes the form

$$
u_{t}=D u_{x x}, \quad 0 \leq x \leq L, \quad 0 \leq t
$$

An example of Inıtral C'onditions (IC) for (6) are

$$
u(x, 0)=\sin (\pi x / L),
$$

and an example of Boundary Conditions (BC) for (6) are

$$
u(0, t)=u(L, t)=0
$$

Boundary conditions of various types, e g flux, periodic, etc are also possible Equation (8) is termed a Dirichlet boundary condition since the dependent variable $u$ is specified at $x=0$ and $L$ If the derivative of the dependent variable with respect to the boundary-value. independent variable is specified, we have a Neumann boundary condution Eg [16]

$$
\frac{\partial u(0, t)}{\partial x}=\frac{\partial u(L, t)}{\partial x}=0
$$

Advection and diffusion can be modeled together by combining equations (5) and (6) into the advection-diffusion equation

$$
u_{t}+a u_{x}=D u_{x x}
$$

which describes the law of conser vation of mass as it applies to the flow of fluids Equation (10) is a continuity equation, i e it states that the mass rate of flow out ol a region of space, such as a control volume, minus the mass rate of flow into the region is equal to the rate at which the fluid mass is being evacuated from the region [17]

A scheme cur rently under much research, for solving problems involving diffusion, is the Preconditıoned Time Differencing method (PTD) The multidimensional heat (diffusion) equation takes the form

$$
\frac{\partial u(\hat{x}, t)}{\partial t}=\alpha \nabla \nabla u(\hat{x}, t)
$$

where $\hat{x}$ is shorthand for the multidimensional vector $\hat{x}=\left(x_{1}, \quad, x_{n}\right)^{T}$ confined to some region $\Omega \quad \hat{x} \in \Omega \subseteq \mathcal{R}^{n}, 0 \leq t \leq t_{\text {final }}<\infty$ and $n>0$ With preconditioning, equation (11) with IC

$$
u(\ddot{x}, 0)=f(\ddot{x})
$$

and well-posed BC's has been solved on vector and parallel computers, using an explicit difference method which is efficient and has a larger stability region than that of the classical explicit Euler schemes The PTD method, used by Garry Rodrigue and Don Wolitzer, uses Forward Euler as predictor, Backward Euler as corıector and the over-relaxed point Jacobi method as the iterative method Explicit methods impose severe constraints on the timestep due to stability considerations Implicit methods on the other hand, do not have such constraints but require a system of simultaneous algebraic equations to be solved at each timestep For pure diffusion, this system is linear since there is no nonlinear reactive term Combining the two methods results in a method which is explicit, has a large stable timestep, and since the dominant operation in an explicit method is the matrix vector multiply, such an 
operation is highly efficient on a pipeline as well as multiprocessing computer architectures [18]

The advection-diffusion equation (10) can be solved with the numerical Finite Difference Method Specifically illustrated here is the Finite Time Central Space method (FTCS) $[19,20]$

$$
\frac{u_{j}^{n+1}-u_{j}^{n}}{\Delta t}+a \frac{u_{j+1}^{n}-u_{j-1}^{n}}{2 \Delta x}=\frac{D}{(\Delta x)^{2}}\left(u_{j+1}^{n}-2 u_{j}^{n}+u_{j-1}^{n}\right)
$$

oI

$$
u_{j}^{n+1}=\mu\left(c_{1} u_{j+1}^{n}+c_{2} u_{j}^{n}+c_{3} u_{j-1}^{n}\right), \quad j=1, \quad, q
$$

whele

$$
\begin{aligned}
c_{1} & =D-a \Delta x / 2, \\
c_{2} & =-2 D+(\Delta x)^{2} / \Delta t, \\
c_{3} & =D+a \Delta x / 2, \\
\mu & =\Delta t /(\Delta x)^{2}
\end{aligned}
$$

With the over-relaxed Jacobi preconditioner described above, the stability regions of the Finite Time Central Space, Upwind Differencing (UW), and Lax-Wendroff (LW) methods have been expanded for the multidimensional diffusion equation (11), the linear advectiondiffusion equation (10), and the nonlinear Burgers' equation [21]

$$
u_{t}+u u_{x}=\alpha u_{x x}
$$

FTCS, UW and IW explicit methods for solving (16), are given in stencil form by

$$
u_{j}^{n+1}=\frac{\gamma_{j}^{n} \Delta t}{\Delta x^{2}}\left(u_{j+1}^{n}-2 u_{j}^{n}+u_{j-1}^{n}\right)+u_{j}^{n}-\frac{u_{j}^{n} \Delta t}{2 \Delta x}\left(u_{j+1}^{n}-u_{j-1}^{n}\right),
$$

where

$$
\begin{array}{ll}
\gamma_{j}^{n}=\alpha, & \text { FTCS, } \\
\gamma_{j}^{n}=\alpha+u_{j}^{n}(\Delta x / 2), & \text { UW }, \\
\gamma_{j}^{n}=\alpha+\left(u_{j}^{n}\right)^{2}(\Delta t / 2), & \text { LW }
\end{array}
$$

Similarly, implirit methods are.

$$
u_{j}^{n+1}=\frac{\gamma_{j}^{n} \Delta t}{\Delta x^{2}}\left(u_{j+1}^{n+1}-2 u_{j}^{n+1}+u_{j-1}^{n+1}\right)+u_{j}^{n+1}-\frac{u_{j}^{n} \Delta t}{2 \Delta x}\left(u_{j+1}^{n+1}-u_{j-1}^{n+1}\right)
$$

The implicit methods (19) have been preconditioned and over-relaxed to produce the PTD computations reported in [21]

PTDs can be applied to advection-diffusion equations which include additional terms making the equation nonlinear The reaction operator ${ }_{i} R$ in equation (4) is generally nonlinear and couples together the reaction-transport PDEs for all species involved Thus, a reactive flow simulation leads to a large system of coupled, nonlinear PDEs

Equation (4) represents a conser vation law for each chemical species ${ }_{i} u$, if we assume that no molecules are created or destroyed, then we have equation (3) for each species On the other hand, if we assume only that no atoms are created or destroyed, but allow molecules to react, we have equation (4)

These equations are often very stiff, placing a severe limitation on the stepsize of an explicit scheme due to stability, which forces the use of an implicit scheme The problem with an implicit scheme, however, is that a nonlinear system must be solved at each step Preconditioned time differencing has yielded stable, explicit methods for dealing with these other wise unwieldy systems Equation (4) has been solved with Jacobi and Gauss-Seidel preconditioners applied to the backward Eules method, and the Jacobi preconditioner applier to the trapezoid rule [22]

$$
\hat{y}^{n+1}=\frac{1}{2} \Delta l\left(\hat{f}^{n+1}+\hat{f}^{n}\right)+\hat{y}^{n}
$$


Equation (4) has also been solved with the Newton preconditioner applied to the backward Euler and trapezoid rules [23, 10] An implicit scheme proposed by Stott and Harwood [24] is an Euler backward scheme in which an initial estimate of the solution is used as the first approximation in a Newton-Raphson iteration to the fully implicit solution The Euler backward scheme may be written

$$
\hat{y}^{n+1}-\hat{y}^{n}-\Delta t \hat{f}\left(\hat{y}^{n+1}\right)=0
$$

The method chosen here to solve for $y^{n+1}$ is to carry out a Newton-Raphson iteration given by

$$
\dot{y}^{n+1, m+1}=\ddot{y}^{n+1, m}+\left(I-\Delta t\left(\frac{\partial \hat{f}}{\partial \hat{y}}\right)^{n+1, m}\right)^{-1}\left(-\ddot{y}^{n+1, m}+\Delta t \hat{f}\left(y^{n+1, m}\right)+\hat{y}^{n}\right),
$$

where the superscript $m$ denotes the item evaluated at the $m$ th iteration, $I$ is the ideritity matrix and $\partial \hat{f} / \partial \hat{y}$ is the Jacobian matrix of derivatives The initial value for the iteration, $\hat{y}^{n+1,0}$, is calculated from a linearized approximation to the implicit backward method

$$
\frac{\hat{y}^{n+1,0}-\hat{y}^{n}}{\Delta t}=\hat{f}\left(\grave{y}^{n}\right)+\left(\frac{\partial \hat{f}}{\partial \grave{y}}\right)^{n}\left(\hat{y}^{n+1,0}-\hat{y}^{n}\right)
$$

In the initial estimate to $\hat{y}^{n+1}$ (equation 23) and in each iteration step (equation 22), a matrix inversion is avoided by approximating the matrix by its diagonal It is found that it is necessary to make only a small number of iterations (typically three) to get a very close approximation to the Eules backward solution The method $(22,23)$ is a fully implicit scheme The method investigated in this thesis is a preconditioned scheme not a fully implicit scheme 


\section{$3 \quad$ Preconditioned Time Differencing}

Preconditioned time differencing (PTD) is a methodology used to constuuct explicit time stepping schemes with large stable timestep intervals PTD begins with an implicit time stepping scheme and applies an iterative solution technique to the resulting simultaneous algebraic system Rather than iterate to convergence, PTD uses a fixed number of iterations of the iterative solution technique in a classic Predictor-Corrector fashion

Predictor-Coriector schemes can be understood as follows Suppose that we wish to solve the standard Initial Value Problem (IVP) by an implicit Linear Multistep Method (LMM) Then at each step we have to solve for $\hat{y}^{n+k}$ the implicit system

$$
\hat{y}^{n+k}+\sum_{j=0}^{k-1} \alpha_{j} \hat{y}^{n+j}=h \beta_{k} \hat{f}\left(\hat{y}^{n+k}, t_{n+k}\right)+h \sum_{j=0}^{k-1} \beta_{j} \hat{f}^{n+j}
$$

We normally do this by the fixed point iteration

$$
\begin{gathered}
y^{n+k, s+1}+\sum_{j=0}^{k-1} \alpha_{j} \hat{y}^{n+j}=h \beta_{k} \hat{f}\left(\hat{y}^{n+k, s}, t_{n+k}\right)+h \sum_{j=0}^{k-1} \beta_{j} \hat{f}^{n+j}, \\
\hat{y}^{n+k, 0} \text { al bitraly, } \quad s=0,1,
\end{gathered}
$$

which will converge to the unique solution of (24) provided that

$$
h<\frac{1}{\left|\beta_{k}\right| L}
$$

where $L$ is a Lipschitz constant of $f$ with respect to $y$ For non-stiff problems, this restriction on $h$ is not significant, in practice, considerations of accuracy put a much more restrictive constraint on $h$ Although (25) will converge for arbitrary $y^{n+k, 0}$, each iteration calls for one evaluation of the function $J$, and computation can obviously be saved if we can piovide as good a guess as possible for $y^{n+k, 0}$ This is conveniently done by using a separate explcct LMM to provide the initial guess $\hat{y}^{n+k, 0}$ We call this explicit method the predictor and the implicit method (24) the corrector, the wo together comprise a Predactor-Corrector $(P()$ pair [14]

If an appropriate initial iterate is used, the inuplicit method's onder of accuracy will be preserved [14] In addition, the resulting time-differencing seheme typically inherits an expanded stable time step interval from the parent implicit method [22]

For illustration, consider the system of linear ODEs with constant coefficients

$$
\frac{d \hat{u}}{d t}=\hat{f}(\dot{u}, t)=A \hat{u}
$$

The implicit Euler method, applied to this ODE systern, takes the fom

$$
\begin{aligned}
u^{n+1} & =u^{n}+\Delta t \hat{f}\left(\hat{u}^{n+1}, t_{n+1}\right), \\
\hat{u}^{n+1}-\Delta t \hat{f}\left(\ddot{u}^{n+1}, t_{n+1}\right) & =u^{n} \\
\ddot{u}^{n+1}-\Delta t A \ddot{u}^{n+1} & =\hat{u}^{n} \\
(I-\Delta t A) \dot{u}^{n+1} & =\hat{u}^{n}
\end{aligned}
$$

and defines a system of simultaneous algebraic equations which must be solved for each timestep An iter ative method for solving this linear system defines a splitting $(I-\Delta t A)=$ $M-N$ Substitute this splitting into (28) to get

$$
\begin{aligned}
(M-N) \dot{u}^{n+1} & =u^{n}, \\
M u^{n+1} & =N u^{n+1}+u^{n}
\end{aligned}
$$


Iterating $\hat{u}^{n+1}$ in (29) produces

$$
M \hat{u}^{n+1, s+1}=N \ddot{u}^{n+1, s}+\grave{u}^{n}, \quad s=0, \quad, m
$$

If $M$ is chosen to be a diagonal matrix, its inverse is easy to compute Multiplying equation (30) by $M^{-1}$ yields

$$
\hat{u}^{n+1, s+1}=M^{-1} N u^{n+1, s}+M^{-1} \hat{u}^{n}, \quad s=0, \quad, m
$$

In a PTD method, the number of iterations $m$ is assumed fixed Thus, $i^{n+1}=\hat{u}^{n+1, m}$ Depending upon the choice of preconditioner $M$, this will often produce an explicit scheme For example, using the point-Jacobi preconditioner $(M=\operatorname{diag}(I-\Delta t A))$ and one iteration $(m=1)$ gives

$$
\ddot{u}^{n+1}=M^{-1} N \hat{u}^{n+1,0}+M^{-1} \ddot{u}^{n}
$$

which (given an explicit value for the initial iterate $\imath^{n+1,0}$ ) defines a tıuly explicit time stepping process

PTD has been applied to linear and nonlinear advection-diffusion problems $[18,21]$ and nonlinear chemical kinetics problems $[25,10]$ The main obstacle in formulating a unified algorithm for a full advection-diffusion-1eaction equation is the choice of initial iterate (or predicted value) The use of an explicit time differencing method (i e explicit Eules) was vital in the advection-diffusion case (see $[18,21])$ but was detrimental to stability in the case of chemical reactions, and led to an unstable algorithm (note the "identity predictor" used in [10] or the simple extrapolation used in [25]) Helc, we investigate the combination of PTD approaches for both chemistry and transport The proposal is to ignore the presence of chemistry during the prediction step this will provide the necessary accuracy to the spatial derivatives while not interfering with the stability of the reaction systern This algorithm is described in the next section 


\section{The Algorithm}

The algorithm takes on a very clear PC flavor, the explicit Euler method is used in the predictor step (ignoring the presence of chemistry) and the Jacobi splitting is applied to the implicit Euler method for the corrector step An overrelaxation step $[18,21]$ is peiformed after the corrector step

'To illustrate the algoithm in general terms, assume that equation (4) has been spatially discretized via the Numerical Method Of Lines [16] approach

$$
\frac{\partial\left({ }_{i} \ddot{u}\right)}{\partial t}={ }_{i} f\left({ }_{1} u, \quad,{ }_{N} \grave{u}, t\right)=(A)_{i} \hat{u}+{ }_{i} \hat{R}\left({ }_{1} \dot{u}, \quad,{ }_{N} \hat{u}, t\right), \quad i=1, \quad, N,
$$

where $A$ is a $q \times q$ matrix derived fiom the spatial discretization scheme, ${ }_{i} \hat{R}$ is the spatial discretization of the reaction source term opelator, and ${ }_{i} \hat{u}=\left({ }_{i} u_{1}, \quad,{ }_{i} u_{q}\right)^{T}$ is the (spatially) discretized approximation to ${ }_{i} u(x, t)$ The notation ${ }_{i} u_{j}^{n}$ will be used to denote the discrete numerical approximation to $i u\left(x_{j}, t_{n}\right)$, i e , the concentration of chemical species $i$ at spatial point $j$ and time point $n$ In other wolds, equation (33) is shorthand for the spatially discretized system of PDEs

$$
\left(\begin{array}{c}
{ }_{i} u_{1} \\
{ }_{i} u_{2} \\
{ }_{i} u_{q}
\end{array}\right)=A\left(\begin{array}{c}
{ }_{i} u_{1} \\
{ }_{i} u_{2} \\
\\
{ }_{i} u_{q}
\end{array}\right)+\left(\begin{array}{cc}
{ }_{i} R_{1}\left({ }_{1} u_{1},\right. & \left.,{ }_{N} u_{1}, t\right) \\
i R_{2}\left({ }_{1} u_{2},\right. & \left.,{ }_{N} u_{2}, t\right) \\
& \\
{ }_{i} R_{q}\left({ }_{1} u_{q},\right. & \left.,{ }_{N} u_{q}, t\right)
\end{array}\right), \quad i=1, \quad, N
$$

The explicit Euler prediction step (ignoring the presence of chemistry) is given by

$$
{ }_{i} \hat{u}^{n+1}=(I+\Delta t A)_{i} \hat{u}^{n},
$$

so that

$$
{ }_{i} u^{n+1,0}=(I+\Delta t A)_{i} u^{n}
$$

will be the predicted value

The preconditioned implicit step is derived from the implicit Euler method Begin by applying Backward Euler (BE) to equation (33)

$$
\begin{aligned}
{ }_{i} \dot{u}^{n+1} & ={ }_{i} u^{n}+\Delta t{ }_{i} f\left({ }_{1} \ddot{u}, \quad,{ }_{N} \hat{u}, t\right) \\
& ={ }_{i} \hat{u}^{n}+\Delta t\left[(A)_{i} \ddot{u}^{n+1}+{ }_{i} \hat{R}\left({ }_{1} \ddot{u}^{n+1}, \quad,{ }_{N} u^{n+1}, t_{n+1}\right)\right] \\
& ={ }_{i} \ddot{u}^{n}+\Delta t(A)_{i} \hat{u}^{n+1}+\Delta t\left[{ }_{i} \hat{R}\left({ }_{1} \hat{u}^{n+1}, \quad,{ }_{N} u^{n+1}, t_{n+1}\right)\right], \\
{ }_{i} \dot{u}^{n+1}-\Delta t(A)_{i} i^{n+1} & ={ }_{i} \dot{u}^{n}+\Delta t\left[{ }_{i} \hat{R}\left({ }_{1} u^{n+1}, \quad,{ }_{N} \hat{u}^{n+1}, t_{n+1}\right)\right], \\
(I-\Delta t A)_{i} \ddot{u}^{n+1} & ={ }_{i} \ddot{u}^{n}+\Delta t\left[{ }_{i} \hat{R}\left({ }_{1} \hat{u}^{n+1}, \quad,{ }_{N} \hat{u}^{n+1}, t_{n+1}\right)\right]
\end{aligned}
$$

The Jacobi splittings (linear [21] and nonlinear [10]) can be applied to this equation to obtain

$$
(M)_{i} \ddot{u}^{n+1,1}=(N)_{i} \hat{u}^{n+1,0}+{ }_{i} \ddot{u}^{n}+\Delta t\left[{ }_{i} \hat{R}\left({ }_{1} i^{n+1,0}, \quad,{ }_{i} \hat{u}^{n+1,1}, \quad,{ }_{N} \hat{u}^{n+1,0}, t_{n+1}\right)\right],
$$

where $(I-\Delta t A)=M-N, M=\operatorname{diag}(I-\Delta t A),{ }_{i} \hat{u}^{n+1,0}$ denotes the predicted value of ${ }_{i} u^{n+1}$, and ${ }_{i} u^{n+1,1}$ denotes the corrected value of ${ }_{i} u^{n+1}$

Chemical kinetic ODEs are used as the source terms in conservation equations and cornmonly take the form $[26,22]$ (scalar equation shown for simplicity)

$$
\frac{d_{i} u}{d l}={ }_{i} R(u, t)={ }_{i} P(u, t)-{ }_{i} \hat{L}(u, t)_{i} u-{ }_{i} \bar{L}(u, t)_{i} u^{2}
$$

where ${ }_{i} P,{ }_{i} \hat{L},{ }_{i} \bar{L}$ are nonnegative quantities representing chemical production and loss rates Important to note is that the production and loss rates, while functions of $u$, do not depend 
on the component ${ }_{i} u$ This allows ${ }_{i} u^{n+1,1}$ to be computed as the solution to a quadratic equation, which is essentially an explicit process [10]

In general, the $i$ th component equation in the PDE system will involve a nonzero term of the form ${ }_{i} \bar{L}(u, t)_{i} u^{2}$ and will therefore be nonlinear in ${ }_{i} u$, so that (in the case of equation 38)

$$
(M)_{i} \hat{u}^{n+1,1}=(N)_{i} \ddot{u}^{n+1,0}+{ }_{i} \ddot{u}^{n}+\Delta t\left[\left({ }_{i} \hat{P}^{n}\right)-\left({ }_{i} \ddot{L}^{n}\right)\left({ }_{i} u^{n+1,1}\right)-\left({ }_{i} \bar{L}^{n}\right)\left({ }_{i} u^{n+1,1}\right)^{2}\right],
$$

where ${ }_{i} \hat{R}(u, t), i_{i} \hat{P}(u, t),\left({ }_{i} \hat{L}^{n}\right)\left({ }_{i} u^{n+1,1}\right)$, and $\left({ }_{i} \bar{L}^{n}\right)\left({ }_{i} u^{n+1,1}\right)^{2}$ are vectors of length $q$, the cumbersome notation $\left({ }_{i} \hat{L}^{n}\right)\left({ }_{i} u^{n+1,1}\right)$ denotes

$$
\left(\begin{array}{c}
\left.{ }_{i} \hat{L}_{1}^{n}\right)\left({ }_{i} u_{1}^{n+1,1}\right) \\
\left.{ }_{i} \hat{L}_{2}^{n}\right)\left({ }_{i} u_{2}^{n+1,1}\right) \\
\\
\left.{ }_{i} \ddot{L}_{q}^{n}\right)\left({ }_{i} u_{q}^{n+1,1}\right)
\end{array}\right)
$$

and $\left({ }_{i} \bar{L}^{n}\right)\left({ }_{i} u^{n+1,1}\right)^{2}$ denotes

$$
\left(\begin{array}{c}
\left({ }_{i} \bar{L}_{1}^{n}\right)\left({ }_{i} u_{1}^{n+1,1}\right)^{2} \\
\left.{ }_{i} \bar{L}_{2}^{n}\right)\left({ }_{i} u_{2}^{n+1,1}\right)^{2} \\
\\
\left({ }_{i} \bar{L}_{q}^{n}\right)\left({ }_{i} u_{q}^{n+1,1}\right)^{2}
\end{array}\right)
$$

Equation (40) is a set of independent quadratıc equations, earh of which can be solved for $i u_{j}^{n+1,1}$ in tems of $i u_{j}^{n+1,0}$ and known quantities, leading to an explirit calculation

If the PDF is linear in $i u$, as our test problem will be, then

$$
\begin{aligned}
{ }_{i} R(u, t) & ={ }_{i} P(u, t)-{ }_{i} \hat{L}(u, t)_{i} u \\
(M T)_{i} u^{n+1,1} & =(N)_{i} \hat{u}^{n+1,0}+{ }_{i} u^{n}+\Delta t\left[\left({ }_{i} \hat{P}^{n}\right)-\left({ }_{i} \hat{I}^{n}\right)\left({ }_{i} u^{n+1,1}\right)\right]
\end{aligned}
$$

Since $M$ is a diagonal matrix, its inverse is easily computable and we get a set of independent linear equations

The last step is to perform an oventelaxation, which is given by

$$
{ }_{i} \hat{u}^{n+1}=(3 / 8)\left({ }_{i} u^{n}\right)+(5 / 8)\left({ }_{i} u^{n+1,1}\right)
$$

'The particular choice of overselaxation constants comes from $[18,21]$ and was necessary for accuracy and stability as applied to advection-diffusion pıoblems

Thus, the proposerl algorithm takes the form

1 Set ${ }_{i} \hat{u}^{n+1,0}=(I+\Delta t A)_{i} \hat{u}^{n}$

2 Solve $M_{i} \ddot{u}^{n+1,1}=N_{i} \hat{u}^{n+1,0}+{ }_{i} \hat{u}^{n}+\Delta t\left[{ }_{i} \dot{R}\left({ }_{1} \ddot{u}^{n+1,0}, \quad,{ }_{i} \hat{u}^{n+1,1}, \quad,{ }_{N} \ddot{u}^{n+1,0}, t_{n+1}\right)\right]$ for $i \hat{u}^{n+1,1}$

3 Set ${ }_{i} \hat{u}^{n+1}=(3 / 8)\left({ }_{i} \hat{u}^{n}\right)+(5 / 8)\left({ }_{i} \hat{u}^{n+1,1}\right)$

Note that if we eliminate the reactive terms, ${ }_{i} P^{n}$ and ${ }_{i} I^{n}$, and the chemical vectolization in equation (44), we recover the linear Jacobi splitting (32) for equation (27) 


\section{The Test Problem}

In this section, a simple reaction-transport problem is developed to study the computational performance of the algorithm from the previous section Let ${ }_{1} u={ }_{1} u(x, t)$ denote the chemical concentration of atomic oxygen $(O)$ and let ${ }_{2} u={ }_{2} u(x, t)$ denote the concentration of molecular ozone $\left(O_{3}\right)$, where $x$ is in units of revolution (rev) and $t$ is in units of day (d) unless indicated otherwise The concentration of molecular oxygen $\left(\mathrm{O}_{2}\right.$, denoted by ${ }_{3} u=$ constant) is significantly larger in the earth's atmosphere than atomic oxygen or ozone and is therefore assumed to be constant in this simple numerical simulation The Chapman model for oxygen-containing chemistry has been described elsewhere $[15,22,23,10]$ Chapman chemistry can be modeled by a coupled system of ODEs which can, in turn, be added as a source term to a simple advective-diffusive PDE system to produce

$$
\begin{aligned}
& \frac{\partial_{1} u}{\partial t}+a \frac{\partial_{1} u}{\partial x}=D \frac{\partial^{2}{ }_{1} u}{\partial x^{2}}+{ }_{1} F-\left({ }_{1} L\right)\left({ }_{1} u\right) \\
& \frac{\partial_{2} u}{\partial t}+a \frac{\partial_{2} u}{\partial x}=D \frac{\partial^{2}{ }_{2} u}{\partial x^{2}}+{ }_{2} P-\left({ }_{2} L\right)\left({ }_{2} u\right)
\end{aligned}
$$

where $x \in(0,1)$ and $t \in(0, \infty)$ Periodic boundary conditions will be used (i e ${ }_{i} u(0, t)=$ ${ }_{i} u(1, t)$ ) to mimic the atmosphere The advection and diffusion paraneters $(a$ and $D)$ are assumed constant. The chemical production and loss rates are given by

$$
\begin{aligned}
& { }_{1} P=2\left(k_{3}\right)\left({ }_{3} u\right)+\left(k_{4}\right)\left({ }_{2} u\right) \\
& { }_{2} P=\left(k_{1}\right)\left({ }_{3} u\right)\left({ }_{1} u\right) \\
& { }_{1} L=\left(k_{1}\right)\left({ }_{3} u\right)+\left(k_{2}\right)\left({ }_{2} u\right) \\
& { }_{2} I=\left(k_{2}\right)\left({ }_{1} u\right)+k_{4}
\end{aligned}
$$

with initial conditions ${ }_{1} u=10^{6} \mathrm{~mol}$ and ${ }_{2} u=10^{12}$ mol, and with constant values ${ }_{3} u=$ $37 \times 10^{16} \mathrm{~mol}, k_{1}=163 \times 10^{-16} \mathrm{~mol}^{-2} \mathrm{~s}^{-1}$ and $k_{2}=466 \times 10^{-16} \mathrm{~mol}^{-2} \mathrm{~s}^{-1}$ The time varying rate coefficient functions (which model chemical reactions driven by the absorption of sunlight) are given by

$$
k_{j}(x, t)=\left\{\begin{array}{ll}
\exp \left(\frac{a_{j}}{\cos (k x-\omega t)}\right) & \text { if } \cos (k x-\omega t)<0, j=3,4 \\
0 & \text { otherwise }
\end{array}, \quad j=\right.
$$

with $a_{3}=2262, a_{1}=7601, \omega=2 \pi /(86,400 \mathrm{~s}), k=2 \pi / \mathrm{rev}$, and the $k_{j}$ are in units of mol $1^{-1} \mathrm{~s}^{-1}$ Note that the constant $86,400 \mathrm{~s}$ is one day, so that the time varying reaction rate coefficient functions $\left(k_{3}\right.$ and $k_{4}$ ) have $24 \mathrm{~h}$ period $T$ These functions are also periodic in $x$ ( $\mathrm{i} \mathrm{e}$ one and only one point in the interval experiences a sunrise/sunset at any given moment) Note also that the wavelength $\lambda$ of these functions is one revolution, $i \mathrm{e}$ one complete circuit along the earth's equator

This test problem, although tractable in size, carries with it many of the important difficulties seen in larger systems it is nonlinear, it has a nonconstant Jacobian, it is sufficiently stiff, due to the chemistry It therefore provides a reasonable initial test for any numerical technique aimed at reactive flow simulation 


\section{Investigation of the Method}

\subsection{Introduction}

This research consisted of testing the PTD method (in a C progiam called reac c) by comparing its performance against that of the Forward Euler (FE) method (euler c) and the Livermore Solver for Ordinary Differential Equations (LSODE) (chapman f) on the same problem FE was used because it is a typical explicit algorithm, against which to compare our explicit algorithm Also, since our PTD is derived from BE, the PTD is fiust-order, as is FE LSODE was used because it is a well-tested, accurate, adaptive-stepsize family of methods Parameters used in each of the three methods were identical, except where different values were necessary, e g stepsize

\subsection{Stability}

Two stability tests of PTD and FE were performed on a Digital Equipment Corporation $\left(D E C^{\prime}\right)$ Reduced Instruction Set Computer (RISC) architecture, running the ULIRIX 43 Operating System (OS) Data collected for PTD during these runs is presented in table (1) Data collected for FE during these 1 uns is presented in table (2) Output values are tabulated for the spatial and temporal point where the percent enor is the largest for nonzero values of $1 u(x, t)$, i e at the tabulated value of $x$, and $t=t_{\text {final }}$

Test 1 used values of the advection and diffusion parameters which are reasonable for an atmospheric simulation Parameters used were

$$
\begin{aligned}
a & =10^{-4} \\
D & =10^{-5} \\
q & =100 \\
\Delta t & =0300 \mathrm{~s} \quad \mathrm{FE} \\
\Delta t & =5034 \mathrm{~s} \quad \mathrm{PTD} \\
t_{0} & =000 \mathrm{~s} \\
t_{\text {final }} & =100 \mathrm{~d}
\end{aligned}
$$

$t_{0}, t_{\text {final }}$ and $\Delta t$ ale simulation time, as opposed to real time and wall clock time The stability of PTD evidently falls apart between $\Delta t=5034 \mathrm{~s}$ and $\Delta t=5035 \mathrm{~s}$ FE exhibits its instability between $\Delta t=030 \mathrm{~s}$ and $\Delta t=035 \mathrm{~s}$ This is an experimental result not an analytical result. Note that accuracy obviously deteriorates at a much smalles $\Delta t$ than stability does A test run for $\Delta t=5 \mathrm{~s}$ is shown for comparison

Test 2 used values of the advection and diffusion parameters which are much larger, to

\begin{tabular}{|l|l|l|l|l|l|l|}
\hline$a$ & $D$ & $\Delta t / \mathrm{s}$ & $t_{\text {final }}$ & $x$ & $\begin{array}{l}1 u(x, t) \\
\text { (from PTD) }\end{array}$ & $\begin{array}{l}\text { Percent } \\
\text { error }\end{array}$ \\
\hline $10^{-4}$ & $10^{-5}$ & 5000000 & $10 \mathrm{~d}$ & 07 & $702 \times 10^{7}$ & -30 \\
$10^{-4}$ & $10^{-5}$ & 5034000 & $10 \mathrm{~d}$ & 07 & $727 \times 10^{11}$ & $73 \times 10^{5}$ \\
$10^{-4}$ & $10^{-5}$ & 5035000 & $10 \mathrm{~d}$ & 07 & unstable & unstable \\
1 & 1 & 0000050 & $100 \mathrm{~s}$ & 03 & $101 \times 10^{7}$ & 12 \\
1 & 1 & 0000051 & $100 \mathrm{~s}$ & 03 & unstable & unstable \\
\hline
\end{tabular}

Table 1 Stability tests of PTD 


\begin{tabular}{|l|l|l|l|l|l|l|}
\hline$\alpha$ & $D$ & $\Delta t / \mathrm{s}$ & $t_{\text {final }}$ & $x$ & $\begin{array}{l}1 u(x, t) \\
\text { (from FE) }\end{array}$ & $\begin{array}{l}\text { Percent } \\
\text { error }\end{array}$ \\
\hline $10^{-4}$ & $10^{-5}$ & 0300000 & $10 \mathrm{~d}$ & 07 & $996 \times 10^{7}$ & $-58 \times 10^{-3}$ \\
$10^{-4}$ & $10^{-5}$ & 0350000 & $10 \mathrm{~d}$ & 07 & unstable & unstable \\
1 & 1 & 0000049 & $100 \mathrm{~s}$ & 03 & $899 \times 10^{6}$ & $12 \times 10^{-2}$ \\
1 & 1 & 0000050 & $100 \mathrm{~s}$ & 03 & unstable & unstable \\
\hline
\end{tabular}

Table 2 Stability tests of FE

exercise the sensitivity of the algorithms' stability to stepsize Parameters used were

$$
\begin{aligned}
a & =1 \\
D & =1 \\
q & =100, \\
\Delta t & =0000049 \mathrm{~s} \quad \mathrm{FE}, \\
\Delta t & =0000050 \mathrm{~s} \quad \mathrm{PTD}, \\
t_{0} & =000 \mathrm{~s}, \\
t_{\text {final }} & =100 \mathrm{~s}
\end{aligned}
$$

The stability of PTD evidently disappears between $\Delta t=0000050 \mathrm{~s}$ and $\Delta t=0000051 \mathrm{~s}$ The stability of FE degener ates between $\Delta t=0000049 \mathrm{~s}$ and $\Delta t=0000050 \mathrm{~s}$ In r unning this test, with a very small $t_{\text {final }}$, both algorithms initially appeared to be stable up to a certain size $\Delta t$, but for laiger $t_{\text {final }}$ and the same $\Delta t$, they eventually exhibited their instability $\Lambda$ ctually, they were unstable for the smaller $t_{\text {final }}$, the instability simply was not apparent The moderate run of $t_{\text {final }}=100 \mathrm{~s}$ was chosen because it is large enough to allow any significant instability to surface, yet small enough to allow the tests to complete within a reasonable amount of wall clock time. With $a=D=1$, we need a very stnall $\Delta t$, which results in very many steps, which for large $t_{\text {final }}$ implies a very long run time. It is very interesting to note that at $t_{\text {final }}=100 \mathrm{~s}$, FE and PTD were both stable up to $\Delta t \approx 000005$, in agreement with the classical 1esult for the Finite Difference Method (FDM) $[20]$

$$
\Delta t \leq \frac{(1 / q)^{2}}{2 D}=\frac{(1 / 100)^{2}}{21}=000005 \mathrm{~s}
$$

\subsection{Accuracy}

'The accuracy test of PTD was performed on the DEC. RISC machine with the same OS The stepsize was successively halved, from $\Delta t=5000000 \mathrm{~s}$, down to $\Delta t=0156250 \mathrm{~s}$, and the output values were compared against those of LSODE solving the same problem with the same parameters The other parameters used were

$$
\begin{aligned}
a & =10^{-4}, \\
D & =10^{-5}, \\
q & =100 \\
t_{0} & =000 \mathrm{~s}, \\
t_{\text {final }} & =500 \mathrm{~d}
\end{aligned}
$$

With $\Delta t=5000000 \mathrm{~s}$, the output values for P'TD were within $19 \%$ of those for LSODF As the stepsize decreased to $\Delta t=0156250 \mathrm{~s}$, the percent entor decreased below $11 \%$ of those for LSODE It is somewhat discouraging that the accuracy is not a little better, but the difference is probably due to the PTD method not adaptively controlling its stepsize The accuracy clearly inıproves slightly with decreasing stepsize (since the PTl) method is first-order accurate) Data collerted during these runs is presented in table (3) Output 


\begin{tabular}{|l|r|l|l|l|}
\hline$\Delta t / \mathrm{s}$ & $t_{\text {final }}$ & $\begin{array}{l}1 u(x, t) \\
(\mathrm{fiom} \mathrm{PTD})\end{array}$ & $\begin{array}{l}\text { Absolute } \\
\text { error }\end{array}$ & $\begin{array}{l}\text { Percent } \\
\text { error }\end{array}$ \\
\hline 5000000 & $5 \mathrm{~d}$ & $634 \times 10^{7}$ & $-151 \times 10^{7}$ & -192 \\
2500000 & $5 \mathrm{~d}$ & $657 \times 10^{7}$ & $-127 \times 10^{7}$ & -162 \\
1250000 & $5 \mathrm{~d}$ & $676 \times 10^{7}$ & $-109 \times 10^{7}$ & -138 \\
0625000 & $5 \mathrm{~d}$ & $688 \times 10^{7}$ & $-961 \times 10^{6}$ & -123 \\
0312500 & $5 \mathrm{~d}$ & $695 \times 10^{7}$ & $-888 \times 10^{6}$ & -113 \\
0156250 & $5 \mathrm{~d}$ & $699 \times 10^{7}$ & $-849 \times 10^{6}$ & -108 \\
\hline
\end{tabular}

Table 3 Comparison of PTD against LSODE for accuracy

ralues are tabulated for the point where the percent erıo is the largest for nonzero values of ${ }_{1} u(x, t)$, i e at $x=080 \mathrm{rev}$, and $t=t_{\text {final }}$ The LSODE value of ${ }_{1} u(x, t)$ at the same point is $784 \times 10^{7}$

\subsection{Parallel Performance}

The platform used for the parallel study was the Meiko CS-2 Multi-Instruction Multi-Data (MIMD) architecture The CS-2 used here is a 40-Node partition, with one $90 \mathrm{MHz}$ Sparc chip (capable of 180 Megaflop performance) and a local cache memory on each node ('ommunication is accomplished via message-passing using a multistage switching network constructed fiom 8 by 8 ciosspoint switches The network is configured so that the bandwidth between stages remains constant

For the runs presented here, the parameters were

$$
\begin{aligned}
a & =10^{-4}, \\
D & =10^{-5} \\
q & =1000 \\
\Delta t & =500 \mathbf{s} \\
t_{0} & =000 \mathrm{~s} \\
t_{\text {final }} & =100 \mathrm{~d}
\end{aligned}
$$

Although these values may seem small, the scale of the spatial domain $(x \in(0,1))$ dictates small transport constants in order to model an "atmosphere" This lype of integration would be prohibilive with a typical explicit method, since the chemistry would normally limit the maximum stepsize to only a few seconds An application of FE to the chemical ODEs alone yields a maximum stable timestep of $023 \mathrm{~s}$ 'This would require more than 21 times as many steps to reach $t_{\text {final }}=100 \mathrm{~d}$ as PTD and a wall clock time of over $2 \mathrm{~h}$ using the entire 40 Central Processing Unit (CPU) partition

The spatial domain is discretized (using centered differences) into 1000 gridpoints which are divided evenly among the nodes After each timestep, the CPUs must exchange the solution values from the boundary of their local domain before the next timestep can begin Thus, as parallelism increases, communication overhead will increase as well Initially, ${ }_{1} u=$ $10^{6}$ mol and ${ }_{2} u=10^{12}$ mol uniformly over the entire space domain

Figure (1) shows the wall clock time vs the number of nodes for this problem An interesting phenomenon is that performance does not scale well until 12-13 CPUs are used in the run At this point, the speedup becomes superlinear, probably due to relatively low communication overhead coupled with good cache performance This hehavior cau be observed further in figure (2), which depicts the relative performance vs the number of nodes The initial drop in this curve is due to increased message passing causing more overhead The subsequent rise in the curve results from each node having less mesh points, each node's portion of the problem begins to fit into the node's cache The second diop 


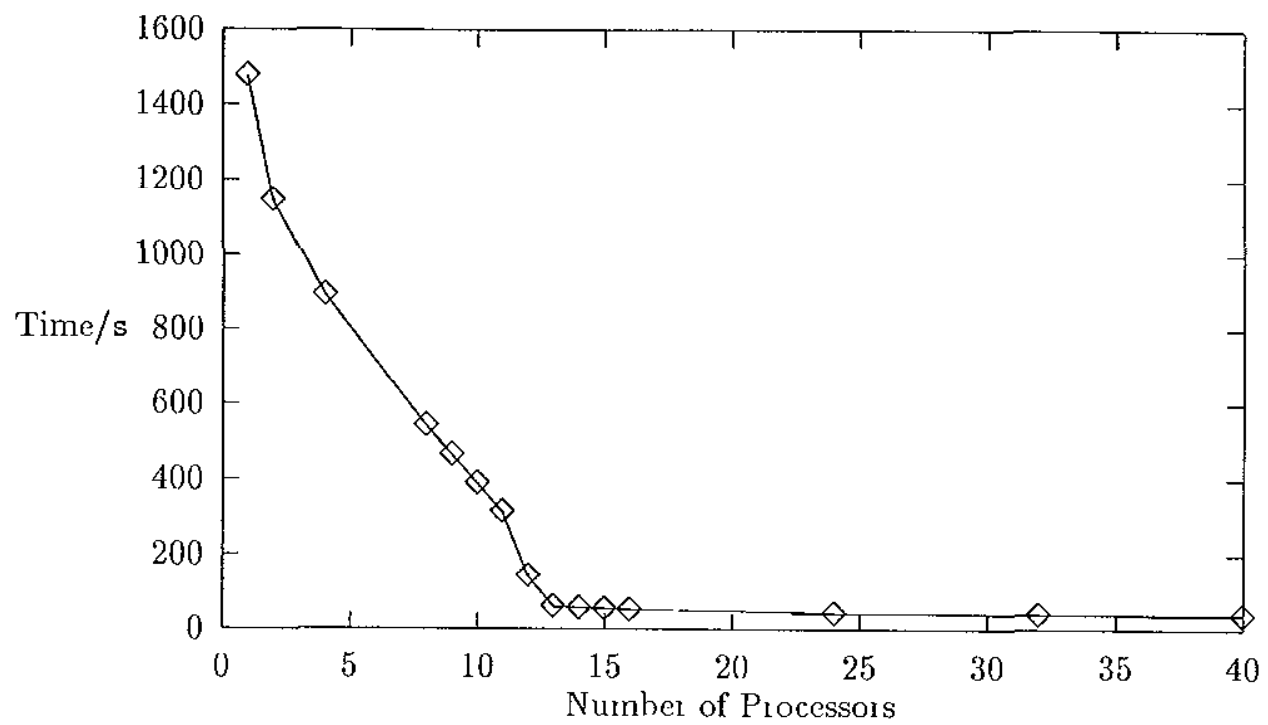

Figure 1 Wall clock time vs number of processing nodes for sample problem

begins when the entire problem fits into (ache, any further division of the problem simply results in more overhead Data collected duning these 1 uns is presented in table (4)

Finally, the parallel performance for an increasing problem size with a constant number of spatial gridpoints per node is depicted Figure (3) shows the wall clock time vs the number of nodes for runs using 250 spatial gridpoints per node There is a slight increase in wall clock time with problem size, but this effect is not very pronounced These results are quite typical for any explicit time stepping method used to numerically solve a PDE system [27]

\subsection{Speed}

A timing comparison of PTD and FE was performed on a Cray C90 Parameters used were

$$
\begin{aligned}
a & =10^{-4} \\
D & =10^{-5} \\
q & =100 \\
\Delta t & =023 \mathrm{~s} \quad \mathrm{FE}, \\
\Delta t & =500 \mathrm{~s} \quad \mathrm{PTD}, \\
t_{0} & =000 \mathrm{~s}, \\
t_{\text {final }} & =500 \mathrm{~d}
\end{aligned}
$$

The goal here was to demonstrate that the stability advantage of $\mathrm{P}^{\prime} \mathrm{CD}$ over FE translates into faster computation The total 1 un time for $\mathrm{FE}$ was $25 \mathrm{~min}, 22 \mathrm{~s}$ The total run time for PTD was 01 min, $07 \mathrm{~s}$ about $1 / 25$ of that for $\mathrm{FE}$

LSODE was not run on the Ciay, as LSODE was the slowest of the three methods Photodissociation reactions occur in the presence of sunlight and their rates of reaction change abruptly at sunset and even more abruptly at sumise At any given time, there is a sunise and a sunset somewhere in the simulation The small stepsizes taken by LSODE as a result of the sharp edges at sumises and sunsets are a product of the dynamic enior control used by ISSODE not of stiffness 


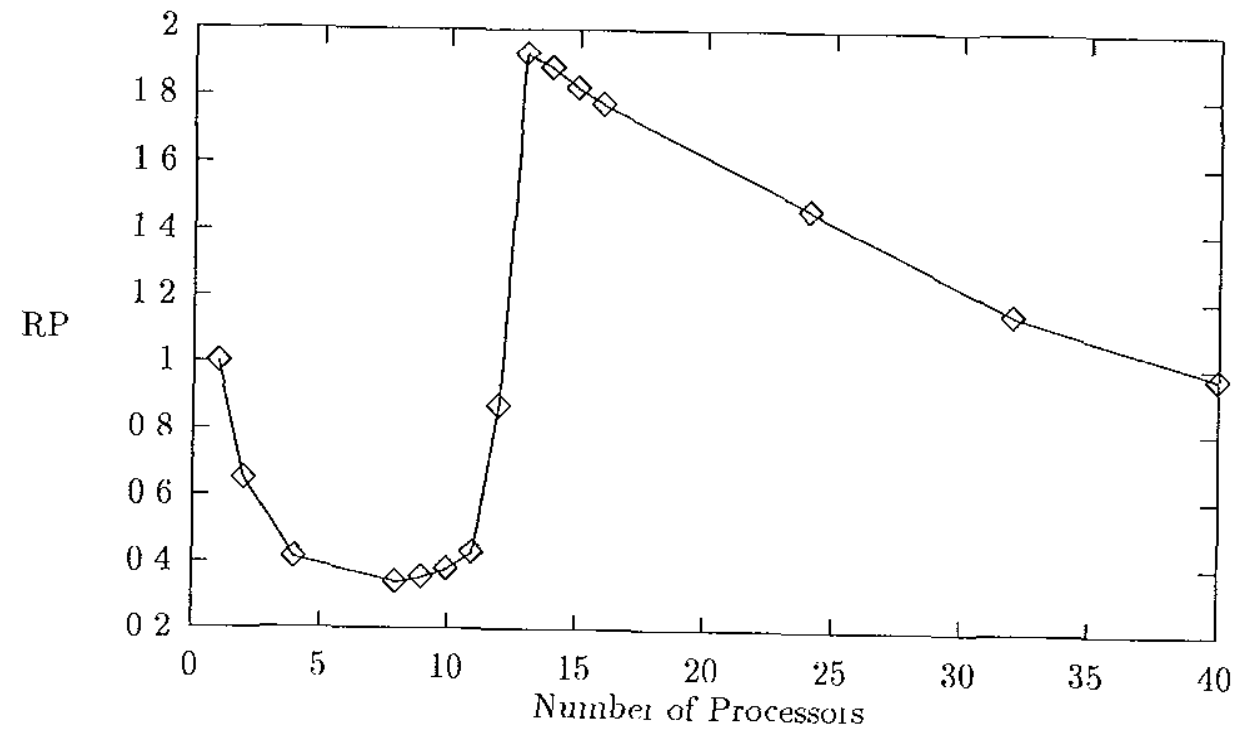

Figure 2 Relative performance vs number of processing nodes for sample picblem

\begin{tabular}{|r|r|}
\hline Number of Processors & Wall Clock Time / \\
\hline 1 & 1476 \\
2 & 1141 \\
4 & 891 \\
8 & 541 \\
9 & 462 \\
10 & 387 \\
11 & 312 \\
12 & 141 \\
13 & 59 \\
14 & 56 \\
15 & 54 \\
16 & 52 \\
24 & 42 \\
32 & 40 \\
40 & 38 \\
\hline
\end{tabular}

Tahle 4 Wall clock times for sample problem runs on the Meiko C'S-2 


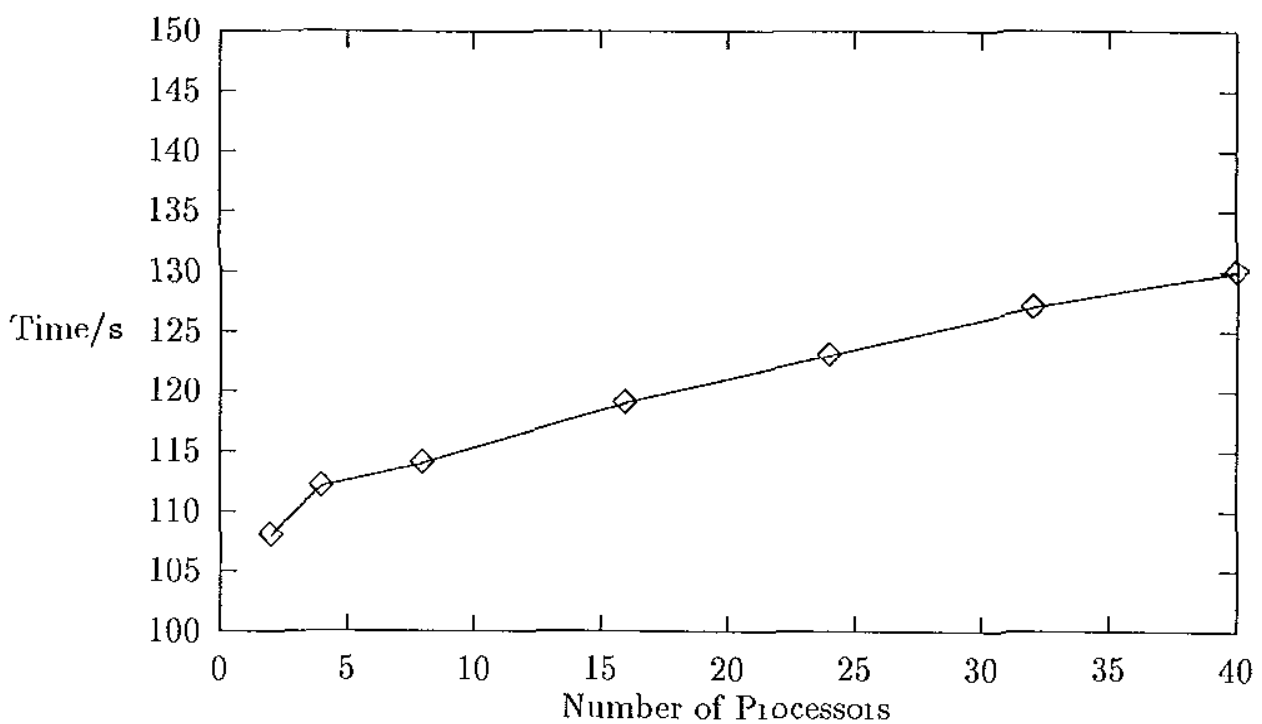

Figure 3 Wall clock time vs processing nodes for 250 spatial gridpoints per processor

\subsection{Output}

The output of the algoithm is the concentrations of the constituent molecular species In our test problem, the outputs are atomic oxygen and molecular ozone Molecular oxygen is nearly constant throughout the simulation Figure (4) shows the change in concentration of molecular ozone at a single point, in one day Figure (5) shows the change in concentration of atomic oxygen at a single point, in one day Figure (6) shows the concentration of atomic oxygen at different pounts, after halt a day has elapsed This wave of atomic oxygen remains pointed loward the sun as the earth turns beneath it Figure (7) shows the concentration of atomic oxygen at difleient points, after three quarters of a day have elapsed Notice that the wave is now at a different place on the equator The simusoidal in the denominator of equation (49) was chosen so that the distance along the equator is numbered from $000 \mathrm{rev}$ to $100 \mathrm{rev}$, beginning at (Heenwich, England, increasing to the west, and time is numbered from $000 \mathrm{~d}$ to $100 \mathrm{~d}$, beginning at inidnight On these scales, Memphis, Tennessee is approximately at $025 \mathrm{rev}$, Midway Islands, Hawaii at $050 \mathrm{rev}$, and Gyangze, China at $075 \mathrm{rev}$ Also note that $025 \mathrm{~d}$ con esponds to $6 \mathrm{am}, 050 \mathrm{~d}$ to noon, and $075 \mathrm{~d}$ to $6 \mathrm{pm}$ 


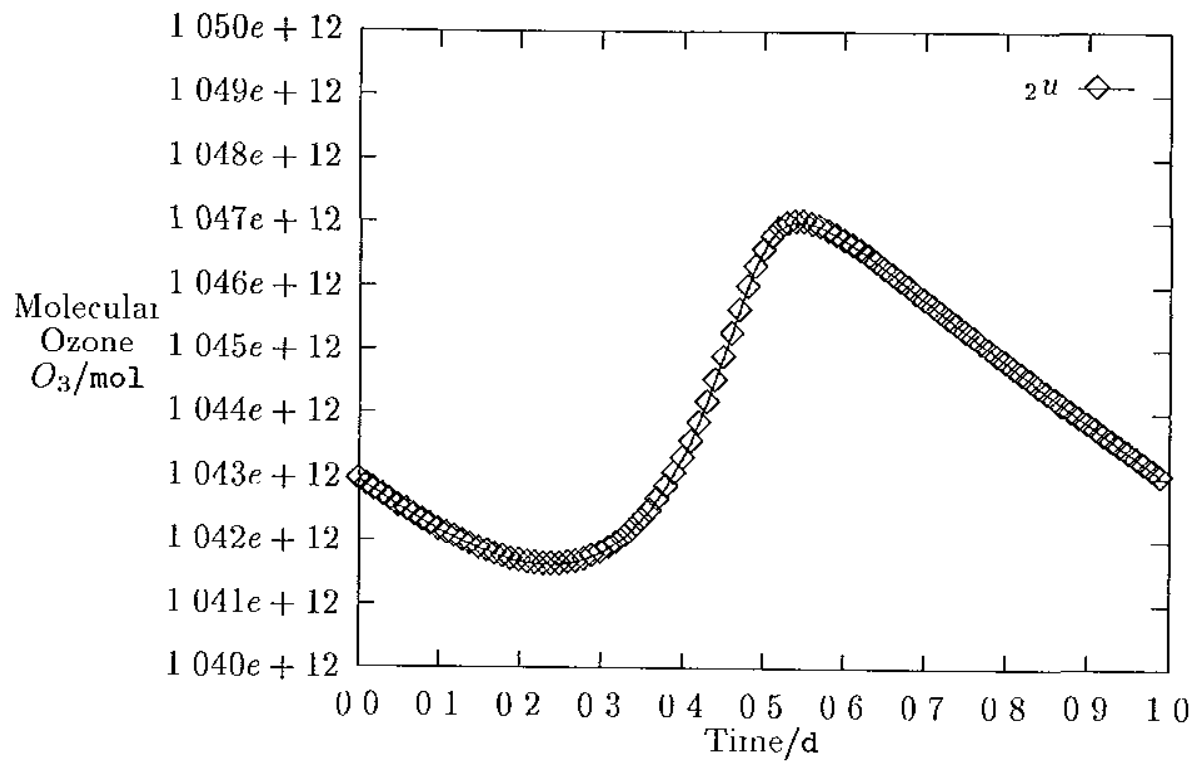

Figure 4 Molecular ozone at a single gridpoint, 000 revolution (corresponding to Greenwich, England), over a period of one day

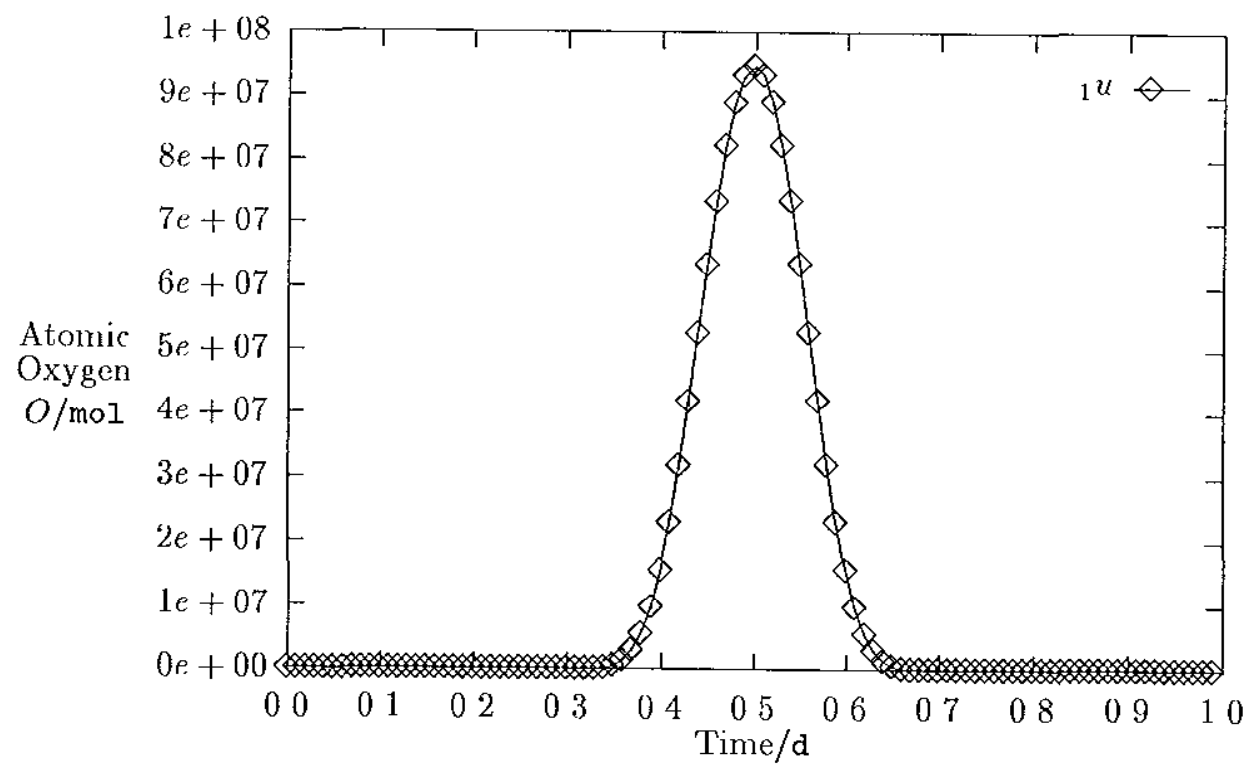

Figure 5 Atomic oxygen at a single gridpoint, 000 revolution (cor esponding to Greenwich, England), over a period of one day Note that at noon in Greenwich, England, the wave of atomic oxygen is directly overhead 


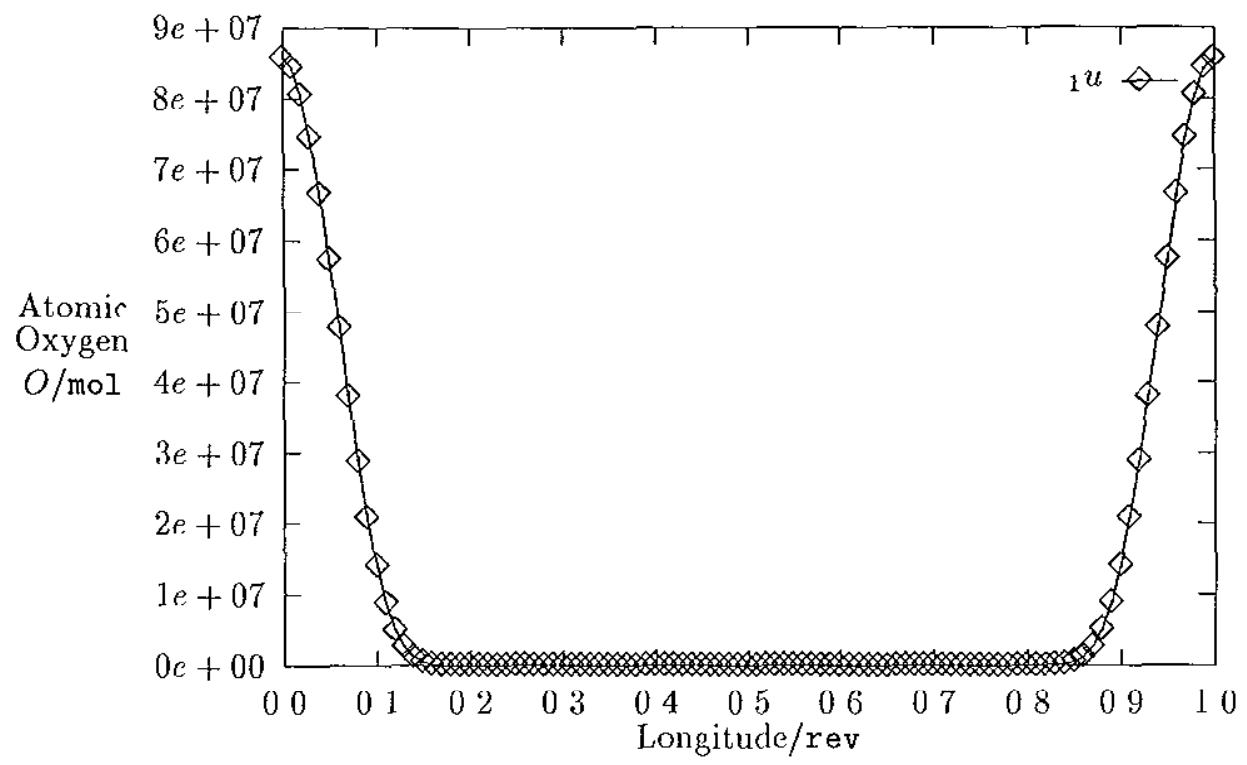

Figure 6 Atomic oxygen along the equator at 050 day (noon) Note that at noon in Greenwich, England, the wave of atomic oxygen is directly overhead

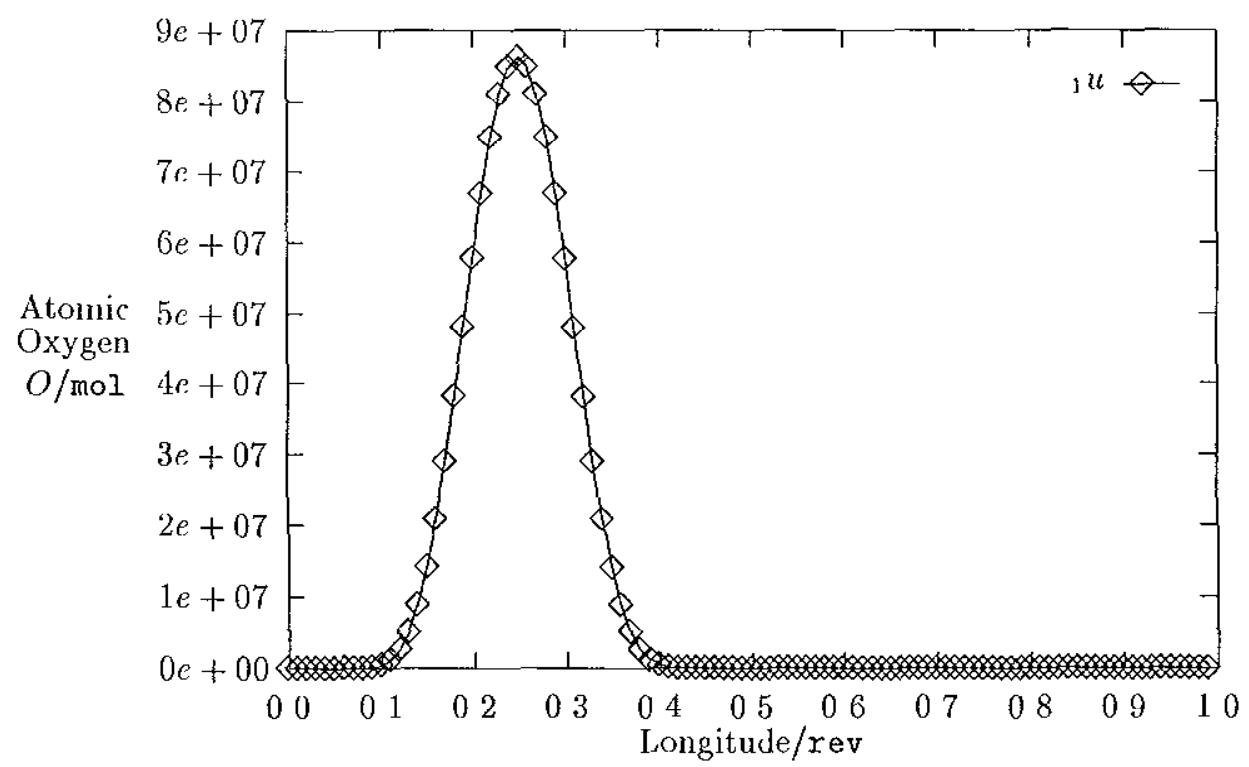

Figure 7 Atomic oxygen along the equator at 075 day $(6 \mathrm{pm})$ Note that at $6 \mathrm{pm}$ in Greenwich, England, the wave of atomic oxygen has moved to Memphis, Tennessee 


\section{Conclusion}

In this investigation we have seen that the stability of the Preconditioned Time Differencing method with parameter values appropiate for practical atmospheric simulations is superior to that of the explicit method, For ward Euler In this case the timestep allowed by stability for the PTD method is about 17 times that of Forward Euler Stability of both methods is nearly equal for large values of the advection and diffusion parameters The accuracy of the PTD method is within $10 \%$ of the accuracy of the fiequently accepted LSODE, using adaptive stepsize On a parallel architecture, the test problem scales well for about 13-39 nodes With a constant number of spatial gridpoints per node, the increase in wall clock time with increasing problem size is small Moreoves, the PT'D method ıuns well on a parallel machine Even in a non-parallel context, while sunning at nearly theis maximum stepsize for stability, PTD requires only about 1/25 the runtime required for FE Hence we have demonstrated stability, accuracy and speed for the PTD method in a reasonable way 


\section{Appendices}

Several adjunct sections are supplied here to explain prerequisite material with which the reader may not alıeady be familiar To see the effect of the periodic motion of the ear th upon photodissociation, the relevance of heat to advection-diffusion equations, or the significance of stift springs in differential equations, it is necessary for the non-physicist reader to have some elementary notions of traveling waves, harmonic motion, thermodynamics, and light Before attempting to comprehend the Chapman chemical mechanism for the distribution of various oxygen species in the atmosphere, it may be prudent for the non-chemist reader to examine some simple principles of rates of reactions and photodissociation, and to be exposed to some essentials of atmospheric chemistıy As the method being discussed and investigated is very mathematical, foundations from several areas of mathematics must be thoroughly grasped before trying to follow the derivation of the method These areas are not extremely advanced but are not fiequently used by computer scientists of all disciplines Farniliarity with these fundamentals will promote the understanding of the core of this thesis Hence these concepts are reviewed here Appendices are also included which cover the hasics of numerical methods and parallel processing 


\section{A Physics Background}

\section{A.1 Traveling Waves}

Wave motion appears in almost every branch of physics We are all familiar with water waves There are also sound waves, as well as light waves, radio waves, and other electromagnetic waves One formulation of the mechanics of atoms and subatomic particles is called wave mechanics Clearly the properties and behaviou of waves are very important in physics For our puiposes we shall need an understanding of the wave properties of light and the periodic variation of the reaction rates of atmospheric gases due to solar radiation In either case our wave is a periodic variation of some physical quantity with respect to time or a spatial dimension or both In this subsection we confine our attention to waves in deformable elastic media These waves, among which ordinary sound waves in ail are one example, might he called mechanucal waves The mathematical properties of this specific type of waves applies to waves of all kinds

Mechanical waves originate in the displacement of some portion of an elastic medium from its nom mosition, causing it to oscillate about an equilibrium position Because of the elastic properties of the medium, the disturbance is transmitted from one point to the next This disturbance, or wave, consequently progresses through the medium It is necessary to have a material medium to tiansmit mechanical waves We do not need such a medium, however, to transmit electromagnetic waves, light passing freely, for example, through the near vacuum of space from the stals

We can distinguish different kinds of mechanical waves by considering how the motions of the particles of matter are related to the direction of propagation of the waves themselves If the inotions of the matter particles conveying the wave are perpendicular to the dilection of propagation of the wave itself, we then have a transuerse wave For example, when a vertical string under tension is set oscillating back and for th at one end, a transverse wave travels down the string, the disturbance moves along the string but the string particles vibrate at iight angles to the direction of propagation of the disturbance

Let us consider a long string stretched in the $x$-direction along which a transvelse wave is traveling At some instant of time, say $t=0$, the shape of the string can be represented by

$$
y=f(x), \quad t=0,
$$

where $y$ is the transverse displacement of the string at the position $x$ Experiment shows that as time goes on, such a wave travels along the string without changing its form, provided intermal fictional losses are small enough At some time $t$ later the wave has traveled a listance $v t$ to the right, where $v$ is the magnitude of the wave velocity, assumed constant The equation of the curve ( $\mathrm{i}$ e shape of the string) at the time $t$ is therefore

$$
y=f(x-v t), \quad t=t
$$

This gives us the same waveform about the point $x=v t$ at time $t$ as we had about $x=0$ at the time $t=0$ Equation (57) is the general equation representing a wave of any shape traveling to the right To describe a particular shape we must specify exactly what the function $f$ is

Let us look more carefully at this equation If we wish to follow a particular part (or phase) of the wave as time goes on, then in the equation we look at a particular value of $y$ (say, the top of the pulse just described) Mathematically this means we look at how $x$ changes with $l$ when $(x-v t)$ has some particulas fixed value We see at once that as $t$ increases $x$ must increase in order to keep $(x-v t)$ fixed Hence, equation (57) does in fart represent a wave traveling to the right (increasing $x$ as time goes on) If we wished to represent a wave traveling to the left, we could wi ite

$$
y=f(x+v t)
$$


for here the position $x$ of some fixed phase $(x+v t)$ of the wave decreases as time goes on The velocity of a particular phase of the wave is easily obtained For a particular phase of a wave traveling to the iight we require that

$$
x-v t=\text { constant }
$$

Then differentiation with respect to time gives

$$
\frac{d x}{d t}-v=0 \quad \text { or } \quad \frac{d x}{d t}=v,
$$

so that $v$ is really the phase velocaty of the wave. For a wave traveling to the left we obtain $-v$, in the same way, as its phase velocity

The general equation of a wave can be interpreted further Note that for any fixed value of the time $t$ the equation gives $y$ as a function of $x$ This defines a curve, and this cunve represents the actual shape of the string at this chosen time It gives us a snapshot of the wave at this time Suppose, on the other hand, we wish to focus our attention on one point of the string, that is, a fixed value of $x$ Then the equation gives us $y$ as a function of the time $t$ This describes how the transverse position of this point on the string changes with time

Let us now consider a particular wavefoum, whose importance will soon become clear Suppose that at the time $t=0$ we have a wavetrain along the stıing given by

$$
y=y_{m} \sin \frac{2 \pi}{\lambda} x
$$

The wave shape is a sine curve. The maximum displacement $y_{n}$ is the amplitude of the sine curve The value of the transverse displacement $y$ is the same at $x$ as it is at $x+\lambda, x+2 \lambda$, etc The symbol $\lambda$ is called the wavelength of the wavetrain and represents the distance between two consecutive points in the wave having the same plase As time goes on let the ware tiavel to the right with a phase velocity $v$ Hence, the equation of the wave at the time $t$ is

$$
y=y_{m} \sin \frac{2 \pi}{\lambda}(x-v t)
$$

Notice that this has the form required for a traveling wave (equation 57)

'The period $T$ is the time required for the wave to travel a distance of one wavelength $\lambda$, so that

$$
\lambda=v T
$$

Putting this relation into the equation of the wave, we obtain

$$
y=y_{m} \sin 2 \pi\left(\frac{x}{\lambda}-\frac{t}{T}\right)
$$

From this form it is cleax that $y$, at any given time, has the same value at $x+\lambda, x+2 \lambda$, etc, as it does at $x$, and that $y$, at any given position, has the same value at the time $t+T$, $t+2 T$, etc, as it does at the time $t$

To reduce equation (64) to a mole compact form, we define two quantities, the wave number $k$ and the angular frequency $\omega$ 'They are given by

$$
k=\frac{2 \pi}{\lambda} \quad \text { and } \quad \omega=\frac{2 \pi}{T}
$$

In terms of these quantities, the equation of a sine wave traveling to the right (positive $(x$-direction) is

$$
y=y_{m} \sin (k x-\omega t)
$$


For a sine wave traveling to the left (negative $x$-direction), we have

$$
y=y_{m} \sin (k x+\omega t)
$$

We will sometimes have need of an inverted sine wave traveling to the right, in which case we have

$$
y=-y_{m} \sin (k x-\omega t)=y_{m} \sin (\omega t-k x)
$$

Comparing equations (63) and (65), we see that the phase velocity $v$ of the wave is given by

$$
v=\frac{\lambda}{T}=\frac{\omega}{k}
$$

If we fix our attention on a given point of the string, say $x=\pi / k$, the displacement $y$ in equation (68) at that point can be witten as

$$
y=y_{m} \sin (\omega t+\phi),
$$

where $\phi$ is called the phase constant, phase shift, or phase angle, and $\phi=-\pi$ in this case Hence, any particular elernent of the sting undergoes what is called simple harmonic motion (SIIM) about its equilibrium position as this wavetrain travels along the string [28]

\section{A.2 Simple Harmonic Motion}

Consides a spring atlached to a wall Let the (horizontal) axis of the spring be chosen as an $x$-axis, and let the origin, $x=0$, coincide with the endpoint of the spring in its normal, unstretched state We assume that the positive $x$-direction points away from the wall In what follows we imagine that we stretch the spring so slowly that it is essentially in equilibrium at all times (acceleration $u=0$ )

If we stretch the sping so that its endpoint moves to a position $x$, the spring will exelt a force on the agent doing the stretching given to a good approximation by

$$
F=-k x,
$$

where $k$ is a constant called the force constant, or spring modulus, or stiffness constant of the spring Equation (71) is the force law for springs The direction of the force is always opposite to the displacement of the endpoint from the origin When the sping is strelched, $x>0$ and $F$ is negative, when the spring is compressed, $x<0$ and $F$ is positive The force exerted by the spring is a restoring force in that it always points toward the origin Real springs will obey equation (71), known as Hooke's law, if we do not stretch them beyond a limited range We can think of $k$ as the magnitude of the force per unit elongation Thus very stiff spings have large values of $k$

A body of mass $m$ attached to an ideal spring of force constant $k$ and fiee to move over a frictionless horizontal surface is an example of a simple harmonic oscillator Note that there is a position (the equilibrium position) in which the spring exerts no force on the body If the body is displaced to the right, the force exerted by the spring on the body points to the left and is given by $F=-k x$ If the body is displaced to the left, the force points to the Iight and is also given by $F=-k x$ In each case the force is a restoring force 'The motion of the oscillating mass is simple harmonic motion (SHM)

Let us apply Newton's second law,

$$
F=m a,
$$

to the motion For the force $F^{\prime}$ we substitute $-k x$ and for the accelcration $a$ we substitute $d^{2} x / d t^{2}$ This gives us

$$
-k x=m \frac{d^{2} x}{d t^{2}}
$$


or

$$
\frac{d^{2} x}{d t^{2}}+\frac{k}{m} x=0
$$

Equation (74) is called the equation of motion of a simple harmonic oscillator [28] Equation (74) can also be witten

$$
m \ddot{x}+k x=0
$$

\section{A.3 Damped Harmonic Motion}

In the previous section we assumed that no frictional forces act on the oscillator If this assumption held strictly, a mass on a spring would oscillate indefinitely Actually, the amplitude of the oscillation gradually decreases to zero as a result of friction The motion is said to be damped by friction and is called damped harmonic motron (DHM) Often the friction arises from ail resistance or internal forces The magnitude of the frictional force usually depends on the speed In most cases of interest the fictional force is proportional to the velocity $\dot{x}$ of the body but directed opposite to it

The equation of motion of the damped simple harmonic oscillaton is given by Newton's second law of motion, $F=m a$, in which $F$ is the sum of the restoring force $-k x$ and the damping force $-c d x / d t$ [28] Here $c$ is a positive constant, called the damping constant [29] We obtain [28]

$$
F=m a
$$

(II

OI

$$
-k x-c \frac{d x}{d t}=m \frac{d^{2} x}{d t^{2}}
$$

$$
\frac{d^{2} x}{d t^{2}}+\frac{c}{m} \frac{d x}{d t}+\frac{k}{m} x=0
$$

Equation (78) can also be written

$$
m \ddot{x}+c \dot{x}+k x=0
$$

\section{A.4 Thermodynamics}

For processes in which heat is involved the laws selating the appropriate inacioscopic quantities (which include pressure, volume, temperature, internal energy, entropy, et al ) form the basis for the science of ther modynamics

The sense of touch is the simplest way to distinguish hot bodies from cold bodies By touch we can arcange bodies in the order of their hotness, deciding that $A$ is hotter $\operatorname{than} B, B$ than $C$, etc We speak of this as our temperature (T) sense [28] More precisely, temperature is a measure of the average inteinal molecular kinetic energy of an object [30] The kelvin (K), unit of thermodynamic temperature, is the fraction 1/273 16 of the thermodynamic. temperat une of the ti iple point of water [28] Heat $(Q)$ is the energy that is transfencd from one object to another because of a difference in temperature [30]

Substances differ from one another in the quantity of heat needed to produce a given rise of temperature in a given mass The ratio of the amount of heat energy $\triangle Q$ supplied to a body to its conresponding temperature rise $\Delta T^{\top}$ is called the heat capacity (C) of the body, i e

$$
C=\frac{\Delta Q}{\Delta T}
$$

'The heat capacity per unit mass of a body, called specific heat $(\sigma)$, is chanacteristic of the material of which the body is composed

$$
\sigma=-\frac{\Delta Q}{m \Delta T}
$$


We properly speak, on the one hand, of the heat capacity of a penny but, on the other, of the specific heat of copper

The transfer of energy arising from the temperature difference between adjacent parts of a body is called heat conduction Consider a slab of material of cross-sectional area $A$ and thickness $\Delta x$, whose faces ale kept at different temperatures We measure the heat $\Delta Q$ that flows perpendicular to the faces in a time $\Delta t$ Experiment shows that $\Delta Q$ is propor tional to $\Delta t$ and to the cross-sectional area $A$ for a given temperature difference $\Delta T$, and that $\Delta Q$ is proportional to $\Delta T / \Delta x$ for a given $\Delta t$ and $A$, providing both $\Delta T$ and $\Delta x$ ate small $\mathrm{I}$ e,

$$
\frac{\Delta Q}{\Delta t} \propto \frac{\Delta T}{\Delta x}
$$

approximately In the limil of a slab of infinitesimal thickness $d x$, across which there is a temperature difference $d T$, we obtain the fundamental law of heat conduction, in which the heat flow $(H)$ is given by

$$
H=-K A \frac{d T}{d x}
$$

Here $H$ is the time tate of heat transfer across the area $A, d T / d x$ is called the temperature gradient, and $K$ is a constant of proportionality called the thermal conductivnty

We shall also need the concept of the density $(\rho)$ of a substance, which is its mass divided by its volume [28]

$$
\rho=\frac{m}{V^{\prime}}
$$

\section{A.5 The Navier-Stokes Equations}

A set of differential equations for the motion of a fluid can be obtained by considening the pressure, gravitational, and viscous forces acting on a fluid element These equations are called the Navier-Stokes equations after L M Navier (1785-1836) and G G Stokes (1819-1903), who are credited with their development These equations for flow of an incompressible fluid in a cattesian coordinate system are

$$
\begin{aligned}
& \rho g_{x}-\frac{\partial p}{\partial x}+\nu \nabla^{2} u=\rho \frac{d u}{d t}, \\
& \rho g_{y}-\frac{\partial p}{\partial y}+\nu \nabla^{2} v=\rho \frac{d v}{d t}, \\
& \rho g_{z}-\frac{\partial p}{\partial z}+\nu \nabla^{2} w=\rho \frac{d w}{d t},
\end{aligned}
$$

whele $g$ is giavitational acceleration, $\rho$ is the mass density, $p$ the pressure, $\nu$ the knetic: viscosity, and $\hat{V}=u \hat{i}+v \hat{j}+w \hat{k}$ the velocity of the fluid It is assumed that the coordinate system is arbitrarily defined Therefore, in the above equations $\rho g_{x}, \rho g_{y}$, and $\rho g_{z}$ are the gravitational forces per unit of volume in the $x, y$, and $z$ direction, respertively [17] for a rigorous derivation of equations (85), see [31]

\section{A.6 The Advection-Diffusion Equation}

Advection is defined as the hor zzontal movement of a mass of ail Convection is defined as the vertucal movement of a mass of air In a 3-D system, a fluid typically moves both horizontally and vertically, to a certain extent Advection and convection are often used inter changeably to refer to movement of ail in any direction Thus, the advection-diffusion equation is often referred to as the convection-diffusion equation in some circles

Diffusion is defined as the intermingling of molecules or other particles as a result of their random thenal motion Cionduction is defined as the transmission of energy by a 
medium which does not involve movement of the medium itself The pure diffusion equation is identical, except for dimensions, to the heat conduction equation

The processes of mass transfer in fluids are modeled by partial differential equations (in particular, the Naviel-Stokes equations) Many of our day-to-day experiences involve the process of mass transfer by convection and diffusion As smoke pours from a tall chimney, the moving ail currents convect or transpoit the smoke away at the speed of the wind, while at the same time the smoke mixes with or diffuses through the air, carrying it until the smoke concentration is so low that we believe the smoke is no longer there, since we cannot see it Pollution disharged to streams and rivers is also transported and mixed in much the same way

In the simplest, 1-D motion ( $x$-dinection only), the fluxes $f_{C}$ due to convection and $f_{D}$ due to diflusion of a quantity being transported across a given section $x$ at time $t$ are

$$
f_{C}=a u, \quad f_{D}=-D u_{x},
$$

where $a$ is the speed of the main fluid (centimeters per second), $u$ is the concentiation of the transported quantity, say smoke or dye or bacteria, per unit volume (grams per cubic centimetes), and $D$ is the diffusion coefficient (centimeters squared per second) Here $f_{D}$ is given by the Fick's rate equation that ielates the diffusive flux to the negative gradient of concentration When the diffusion results from the ıandom vibıation of molecules in a gas or liquid, Fick's law is valid and $D$ is often essentially a constant that can be determined experimentally In the wind above the chimney, the diffusion is likely to icsult from the motion of the turbulent eddies in the wind, Fick's law and constant $D$ are then simplifying assumptions used to gain simple, but approximate, results

An analysis of a slice $\Delta x$ thick through a 1-D stream leads to a conservation equation for the trausported mass The resulting partial differential equation is

$$
u_{t}+a u_{x}=D u_{x x}
$$

and is called the 1-D advection-diffusion equation $[19]$

The $3-\mathrm{D}$ advection-diffusion equation is

$$
\frac{\partial u}{\partial t}+a \nabla u=D \nabla \nabla u
$$

Fol a rigorous derivation of $(88)$, see [32]

\section{A 6.1 Pure Convection}

Suppose that the transpolted quantity is a led dye of strength $u$ that diffuses slowly in water so that the dye cloud has a motion governed essentially by convection when $a$ is fairly large A source of dye of concentration $u_{0}$ is available at a point in a 1-D stream of constant speed a Putting the condinate origin at the source and turning the source on to full strength at time zero lead to a model for $u(x, t)$

$$
\begin{aligned}
& u_{t}+a u_{x}=0, \quad 0<x, \quad 0<t \\
& u(0, t)=u_{0}, \quad 0<t \\
& u(x, 0)=0, \quad 0 \leq x
\end{aligned}
$$

We assume that $u$ and $u_{x}$ approach zero as $x \rightarrow \infty$ for all $t$ Note that we have a model with a first-order partial differential equation Equation (89) is the 1-D pure convection equation (a $\mathrm{k}$ a advection equation) [19] 


\section{A 6.2 Pure Diffusion}

Suppose, in the previous example, that the velocity of the fluid $a$ is very slow so that diffusion dominates the convection 'The model would then be modified to be, for $D>0$,

$$
\begin{aligned}
& u_{t}=D u_{x x}, \quad 0<x, \quad 0<t \\
& u(0, t)=u_{0}, \quad 0<t \\
& u(x, 0)=0, \quad 0 \leq x \\
& |u(x, t)|<\infty
\end{aligned}
$$

with $u$ and $u_{x} \rightarrow 0$ as $x \rightarrow \infty$ for all $t$ Equation (92) is second-order and is the 1-D diffusion equation [19] For a non-ligolous derivation of (92), see [33]

\section{A.6.3 The Heat Equation}

The flow of heat in a conducting solid, with certain simplifying assumptions, results in the same model employing partial differential equations as for pue diffusion (except for dimensions) The 3-D heat equation is

$$
\frac{\partial u}{\partial t}=k \nabla \quad \nabla u
$$

whele

$$
k=\frac{K}{\rho \sigma}
$$

is called the thermal diffustuty, $K(x, y, z, u)$ is the thermal conductanty of the solid mediun, assumed constant here, $\rho$ is the density of the solid (mass per unit volume), also assumed constant, and $\sigma$ is the specafic heat of the material of the solid (heat per unit mass per unit of temperature) For a rigorous derivation of (96), see [19]

\section{A.7 Light}

Light has both wave-like and particle-like properties As a wave, it is a rombination of oscillating electric and magnetic fields perpendicular to each other and to the diection of propagation The distance between consecutive peaks is the wavelength $\lambda$ and the number of complete cycles passing a fixed point in $1 \mathrm{~s}$ is the frequency $\nu$ They are inversely proportional though the relationship

$$
\lambda=\frac{c}{\nu},
$$

where $c$ is the velocity of light in a vacuum

Considered as a particle, the energy $E$ of a quantum of light is

$$
E=h \nu=\frac{h c}{\lambda},
$$

where $h$ is Planck's constant [3] 


\section{B Chemistry Background}

\section{B.1 Rates of Reactions}

Different chemical leactions take place at different rates Some reactions, such as the reaction between $\mathrm{AgNO}_{3}(a q)$ and $\mathrm{KCl}(a q)$ to produce a precipitate of $\mathrm{AgCl}(s)$, seem to occur almost instantaneously, whereas other reactions, such as the reaction between $H_{2}(g)$ and $N_{2}(g)$ to produce $\mathrm{NH}_{3}(\mathrm{~g})$, occur very slowly The reaction rate law, which inust be determined by experiment, tells us how the iate of a reaction depends on the concentration of the reactants and of other added substances, such as catalysts

Consider the decornjosition of hydrogen peroxide in aqueous solution

$$
2 \mathrm{H}_{2} \mathrm{O}_{2}(\mathrm{rq} q) \rightarrow 2 \mathrm{H}_{2} \mathrm{O}(l)+\mathrm{O}_{2}(g)
$$

Because a gaseous product $O_{2}(g)$ forms, we can determine how its concentration increases with time by measuing the increase in pressure above the reaction mixture let us see how we can use this measurement to define a reaction rate

Suppose that we know the concentration of the oxygen gas at some time to be $\left[\mathrm{O}_{2}\right]_{1}$, and suppose we find that at some later time it is $\left[\mathrm{O}_{2}\right]_{2}$ We can wite the change in its concentration as

$$
\Delta\left[O_{2}\right]=\left[O_{2}\right]_{2}-\left[O_{2}\right]_{1}
$$

Because the concentration of the product increases with time, we know that $\Delta\left[\mathrm{O}_{2}\right]$ must be positive We can now define a reacton rate as the average rate at which oxygen is produced, or the change in concentration divided by the elapsed time

$$
\frac{\Delta\left[O_{2}\right]}{\Delta t}
$$

Note that the rate of a reaction is a positive quantity Its units are moles per liter per second (mol $\mathrm{L}^{-1} \mathrm{~s}^{-1}$ or $\mathrm{M}^{-1}$ ), or, more generally, moles per liter per unit time

We can also define a reaction rate by examining how quickly the reactant is consumed Now we are interested in the average number of moles per liter rearted per second Because the concentration of $\mathrm{H}_{2} \mathrm{O}_{2}$ is decreasing, $\Delta\left[\mathrm{H}_{2} \mathrm{O}_{2}\right]$ is a negative quantity, but we can insert a minus sign to nake the reaction rate positive

$$
-\frac{\Delta\left[\mathrm{H}_{2} \mathrm{O}_{2}\right]}{\Delta t}
$$

The relation between the two equations depends on the equation for the reaction In this case, we know the rate at which $\mathrm{O}_{2}$ is produced is equal to one half the rate at which $\mathrm{H}_{2} \mathrm{O}_{2}$ is consumed, because two $\mathrm{H}_{2} \mathrm{O}_{2}$ molecules react for each $\mathrm{O}_{2}$ molecule produced Thus, for the reaction rate $R$, we have

$$
R=-\left(\frac{1}{2}\right)\left(\frac{\Delta\left[H_{2} O_{2}\right]}{\Delta t}\right)=\frac{\Delta\left[O_{2}\right]}{\Delta t}
$$

The rate of the reaction for the decomposition of $\mathrm{N}_{2} \mathrm{O}_{5}(g)$ in

$$
\mathrm{N}_{2} \mathrm{O}_{5}(g) \rightarrow 2 \mathrm{NO}_{2}(g)+\frac{1}{2} \mathrm{O}_{2}(g)
$$

( an be expressed as

$$
R=-\left(\frac{1}{1}\right)\left(\frac{\Delta\left[N_{2} O_{5}\right]}{\Delta t}\right)=\left(\frac{1}{2}\right)\left(\frac{\Delta\left[N O_{2}\right]}{\Delta t}\right)=\left(\frac{1}{1 / 2}\right)\left(\frac{\Delta\left[O_{2}\right]}{\Delta t}\right)
$$

Notice that the numerical factor in the denominator of each expression is the same as the balancing coefficient of the species in the chemical equation 
Thus, for the reaction

$$
2 \mathrm{O}_{3}(g) \rightarrow 3 \mathrm{O}_{2}(g)
$$

we define the rate to be

$$
R=-\left(\frac{1}{2}\right)\left(\frac{\Delta\left[O_{3}\right]}{\Delta t}\right)=\left(\frac{1}{3}\right)\left(\frac{\Delta\left[O_{2}\right]}{\Delta t}\right)
$$

Table (5) gives the numerical values of $\left[\mathrm{N}_{2} \mathrm{O}_{5}\right],\left[\mathrm{NO}_{2}\right]$, and $\left[\mathrm{O}_{2}\right]$ that cor respond to reaction (105), as a function of time at $45^{\circ} \mathrm{C} \quad \mathrm{An}$ inspection of the data in table (5) shows that the reaction rate decreases as $\left[\mathrm{N}_{2} \mathrm{O}_{5}\right]$ decreases For example, the rate of decomposition of $\mathrm{N}_{2} \mathrm{O}_{5}$ over the first 10 minutes of the reaction is

$$
R=-\frac{\Delta\left[N_{2} O_{5}\right]}{\Delta t}=32 \times 10^{-4} \mathrm{~mol} \mathrm{~L} \mathrm{~L}^{-1} \mathrm{~min}^{-1}
$$

whereas the rate over the period $10 \mathrm{~min}$ to $20 \mathrm{~min}$ is

$$
R=24 \times 10^{-4} \mathrm{~mol} \mathrm{~L} \mathrm{~L}^{-1} \min ^{-1}
$$

\begin{tabular}{|r|l|l|l|}
\hline$t / \mathrm{min}$ & {$\left[\mathrm{N}_{2} \mathrm{O}_{5}\right] / \mathrm{M}$} & {$\left[\overline{\mathrm{NO}_{2}}\right] / \mathrm{M}$} & {$\left[\mathrm{O}_{2}\right] / \mathrm{M}$} \\
\hline 0 & $124 \times 10^{-2}$ & 0 & 0 \\
10 & $092 \times 10^{-2}$ & $064 \times 10^{-2}$ & $016 \times 10^{-2}$ \\
20 & $068 \times 10^{-2}$ & $112 \times 10^{-2}$ & $028 \times 10^{-2}$ \\
30 & $050 \times 10^{-2}$ & $148 \times 10^{-2}$ & $037 \times 10^{-2}$ \\
40 & $037 \times 10^{-2}$ & $174 \times 10^{-2}$ & $044 \times 10^{-2}$ \\
50 & $027 \times 10^{-2}$ & $192 \times 10^{-2}$ & $048 \times 10^{-2}$ \\
60 & $020 \times 10^{-2}$ & $208 \times 10^{-2}$ & $052 \times 10^{-2}$ \\
70 & $014 \times 10^{-2}$ & $218 \times 10^{-2}$ & $055 \times 10^{-2}$ \\
80 & $011 \times 10^{-2}$ & $226 \times 10^{-2}$ & $057 \times 10^{-2}$ \\
90 & $008 \times 10^{-2}$ & $232 \times 10^{-2}$ & $058 \times 10^{-2}$ \\
100 & $006 \times 10^{-2}$ & $236 \times 10^{-2}$ & $059 \times 10^{-2}$ \\
\hline
\end{tabular}

Table 5 concentration of $\mathrm{N}_{2} \mathrm{O}_{5}(g), \mathrm{NO}_{2}(g)$ and $\mathrm{O}_{2}(g)$ as a function of time

It is important to realize that, given the initial concentrations in table (5), the concentrations of $\mathrm{NO}_{2}(g)$ and $\mathrm{O}_{2}(g)$ can be calculated from the concentration of $\mathrm{N}_{2} \mathrm{O}_{5}(g)$ at any time

We have noted that the rate of a reaction changes as the reactants ale consumed and the products are formed This change occurs because the rates of chemical reactions usually depend on the concentrations of one or more of the reactants The mathematical equation that gives this dependence is called the rate law of the reaction Many experiments show that the rate of a reaction usually is proportional to the concentrations of the reactants laised to small integer powers Thus, for the case of the thermal decomposition of $\mathrm{N}_{2} \mathrm{O}_{5}$, we will assume that

$$
R \propto\left[\mathrm{N}_{2} \mathrm{O}_{5}\right]^{x}
$$

We can wite this proportionality as an equation by inserting a propontionality constant

$$
R=k\left[\mathrm{~N}_{2} \mathrm{O}_{5}\right]^{x}
$$

The proportionality constant $k$ in a rate law is callerl the rate constant of the reaction

The value of $x$ in equation (112) must be determined experimentally, it is not necessarily the same as the balancing coefficient of $\mathrm{N}_{2} \mathrm{O}_{5}$ in the chemical equation There as no relation 
between the balancing coefficients in a chemical equation and the reaction rate law For example, the rate law for the reaction

$$
2 \mathrm{H}_{2} \mathrm{O}_{2}(a q) \rightarrow 2 \mathrm{H}_{2} \mathrm{O}(l)+\mathrm{O}_{2}(g)
$$

is

$$
R=k\left[\mathrm{H}_{2} \mathrm{O}_{2}\right]
$$

whereas the rate law for the reaction

$$
2 N O B r(g) \rightarrow 2 N O(g)+\mathrm{Br}_{2}(g)
$$

is

$$
R=k[N O B i]^{2}
$$

In practice, rate laws often are determined from data involving leaction rates at the eally stage of reactions Using the method of initıal rates, we measure the rate of a reaction over an initial time interval that is short enough so that the concentrations of the reactants do not vary appleciably from their initial values If we use zeros as subscipts to denote the initial values of the rate and of the various concentrations, then for any reaction described by an equation of the form

$$
a A+b B+c C \rightarrow p r o d u c t s
$$

we can write

$$
R_{0}=k[A]_{0}^{x}[B]_{0}^{y}[C]_{0}^{z}
$$

We can then deternime the values of $x, y$, and $z$ by varying the initial concentration of each reactant in turn, while keeping the other reactant concentrations constant As a simple

\begin{tabular}{|c|c|c|}
\hline$R u n$ & {$\left[\mathrm{~N}_{2} \overline{\mathrm{O}}_{5}\right]_{0} / \mathrm{mol} \mathrm{L}^{-1}$} & $R_{0} / \mathrm{mol} \mathrm{L}^{-1} \mathrm{~h}^{-1}$ \\
\hline 1 & 0010 & 0018 \\
\hline 2 & 0020 & 0036 \\
\hline 3 & 0040 & 0072 \\
\hline
\end{tabular}
example, let's consider the $\mathrm{N}_{2} \mathrm{O}_{5}$ decomposition reaction, in which there is only one reactant

$$
\mathrm{N}_{2} \mathrm{O}_{5}(g) \rightarrow 2 \mathrm{NO}_{2}(g)+\frac{1}{2} \mathrm{O}_{2}(g)
$$

Suppose that we 1 un this reaction using successively doubled concentiations of $N_{2} O_{5}$, and obtain the initial-rate data in table (6) Notice that when the initial concentration, $\left[\mathrm{N}_{2} \mathrm{O}_{5}\right]_{0}$,

Table 6 Initial sate data for reaction $\mathrm{N}_{2} \mathrm{O}_{5}(g) \rightarrow 2 \mathrm{NO}_{2}(g)+\frac{1}{2} \mathrm{O}_{2}(g)$

is increased by a factor of 2 , the initial rate $R_{0}$ also increases by a factor of 2 These results show that $R_{0}$ is directly proportionl to $\left[\mathrm{N}_{2} \mathrm{O}_{5}\right]_{0}$, so we can wite

$$
R_{0}=k\left[N_{2} O_{5}\right]_{0}
$$

showing that $x=1$ in equation (118)

Extensive experimental studies show that in most cases the reaction rate law determined from initial-rate data describes the reaction rate as the reaction proceeds 'Thus, we can drop the subscript zero in equation (120) to obtain the sate law

$$
R=k\left[N_{2} O_{5}\right]
$$

Because $\left[\mathrm{N}_{2} \mathrm{O}_{5}\right]$ is raised to the first power in equation (121), the rate law is said to be a first-order rate law, and the reaction is said to be a first-order reartion We say that the rate law is first-order in $\left[\mathrm{N}_{2} \mathrm{O}_{5}\right]$ 
We can determine the numerical value of the rate constant $k$ in equation (121) by using the tabulated initial-rate data If we substitute the fact that $R_{0}=0072 \mathrm{~mol} \mathrm{~L}^{-1} \mathrm{~h}^{-1}$ when $\left[N_{2} O_{5}\right]_{0}=0040 \mathrm{~mol} \mathrm{~L} \mathrm{~L}^{-1}$, then we obtain

$$
k=\frac{R_{0}}{\left[N_{2} O_{5}\right]_{0}}=18 \mathrm{~h}^{-1}
$$

Notice that the units of the rate constant for a first-order reaction are reciprocal time, i $\mathrm{e}$ time $e^{-1}$

So far we have encountered only first-order reactions Although it will not be demonstrated here, the iate law for the reaction described by

$$
2 \mathrm{NO}_{2}(g) \rightarrow 2 \mathrm{NO}(g)+\mathrm{O}_{2}(g)
$$

is

$$
R=k\left[\mathrm{NO}_{2}\right]^{2}
$$

Because the exponent of $\left[\mathrm{NO}_{2}\right]$ in the rate law is 2 , we say that the reaction rate law is second-order with respect to $\mathrm{NO}_{2}$, and because $\left[\mathrm{NO}_{2}\right]$ is the only concentration that appears in the rate-law expression, we say that the decomposition of $\mathrm{NO}_{2}(g)$ to $\mathrm{NO}(g)$ and $\mathrm{O}_{2}(g)$ is a second-order reaction

We can also detemine a ate laws for reactions involving more than one reactant For example, the reaction

$$
2 \mathrm{NO}_{2}(g)+F_{2}(g) \rightarrow 2 \mathrm{NO}_{2} F(g)
$$

has the reaction rate law

$$
R=k\left[\mathrm{NO}_{2}\right]\left\{F_{2}\right]
$$

with

$$
k=100 \times 10^{-4} \mathrm{M}^{-1} \mathrm{~s}^{-1}
$$

This rate law is first-order in both $\left[\mathrm{NO}_{2}\right]$ and $\left[\mathrm{H}_{2}^{\prime}\right]$, and we say that it is second-order overall berause the sum of the exponents is two Note that the units of $k$ lor a second-order reaction are reciprocal concentration per time, i e $\mathrm{M}^{-1} \mathrm{~s}^{-1}$

More generally, if a reaction rate law is of the form

$$
R=k[A]^{x}[B]^{y}\left[C^{y}\right]^{z},
$$

then the rate law is $x$-order in $[A], y$-order in $[B]$, and $z$-order in [C], with an overall order of $x+y+z \quad[11]$

\section{B.2 Photodissociation}

The principle of photochemical processes can be summarized as follows A certain atmospheric gaseous component $A$ absolbs a given band of solar radiation in the ultraviolet or visible spectıum Due to the energy of the absorbed photon $h \nu, A$ is changed to an excited state $A^{*}$ Because of this energy, the molecule decomposes, or reacts with another species $B$, or fluoresces, or undergoes quenching, or transfers its energy to another species [1] We will consider here only the molecular decomposition or dissociation The process may be symbolized in the following way

$$
\begin{array}{ll}
A+h \nu \rightarrow A^{*} & \text { absorption } \\
A^{*} \rightarrow D_{1}+D_{2}+ & \text { dissociation }
\end{array}
$$

The last step in this sequence is termed a primary photochemical process Wo have

$$
\frac{d\left[A^{*}\right]}{d t}=k_{a}[A]
$$


where $k_{a}$ is the absorption rate of photons The iate $\mathrm{R}$ of the primary photochemical process will be

$$
R=\frac{d\left[D_{1}\right]}{d t}=k_{a} \phi[A],
$$

where $\phi$ is the so-called quantum yzeld Its value is equal to the ratio of the number of $A$ reacted, to that of excited molecules In photochemical reactions, the rate constant is given by the product of the absorption rate and the quantum yield [34]

\section{B.3 The Chapman Mechanism}

The Chapman mechanism for oxygen-containing chemistry has been dealt with previously $[15,22,23,10]$ and was the first attempt (in the late 1930s) to explain the ozone distribution in the stratosphere The Chapman model assumes that oxygen-containing chemistry alone is sufficient to explain ozone distribution In this case, only the chemistry for $\mathrm{O}, \mathrm{O}_{2}$, and $O_{3}$ is included

Let ${ }_{1} u={ }_{1} u(x, t)$ denote the concentration of atomic oxygen $(O)$ and let ${ }_{2} u={ }_{2} u(x, t)$ denote the concentration of inolecular ozone $\left(\mathrm{O}_{3}\right)$ The concentration of molecular oxygen $\left(O_{2}\right.$, denoted by $3 u=$ constant) is significantly larger in the earth's atmosphere than atomic oxygen or ozone and is therefore assumed to be constant in this simple model, as with most atmospheric simulations

The formation of the stratospheric ozone laye can be understood most simply on the basis of a reaction model composed of a minimum set of four elementary processes (a) the attachment of oxygen atoms to moleculas oxygen, leading to the formation of ozone, (b) the destruction of ozone by its icaction with oxygen atoms, (c) the dissociation of oxygen nolecules by solar radiation in the wavelength segion 180-240 nm, and (d) the photodissociation of ozone in the Hartley hand between 200 and $300 \mathrm{~nm}$ The reactions may be written

$$
\begin{aligned}
O+O_{2}+M & \rightarrow O_{3}+M \\
O+O_{3} & \rightarrow 2 O_{2} \\
O_{2}+h \nu & \rightarrow 2 O \\
O_{3}+h \nu & \rightarrow O+O_{2}
\end{aligned}
$$

In reaction (132), $M$ denotes as usial an inet third body, which in the atmosphere is supplied by either nitıogen or oxygen [1]

These reactions have ieaction 1 ate coefficient functions [26] $k_{1}, k_{2}, k_{3}$, and $k_{4}$ respectively The first two reactions are theimal reactions, so $k_{1}$ and $k_{2}$ can he taken as constants The last two reactions, however, are photochemical in nature (involving the absorption of light energy $-h \nu$ ), and change with the rising/setting of the sun Thus, $k_{3}$ and $k_{4}$ have diurnal cycles and valy with time

The ordinary differential system describing the time-evolving behavior of these chemical concentrations subject to the four reactions is taken from Aro [10]

$$
\begin{aligned}
& \frac{d_{1} u}{d t}={ }_{1} P-\left({ }_{1} L\right)\left({ }_{1} u\right) \\
& \frac{d_{2} u}{d t}={ }_{2} P-\left({ }_{2} L\right)\left({ }_{2} u\right),
\end{aligned}
$$

where ${ }_{1} u$ and ${ }_{2} u$ are in units of mol Here, ${ }_{1} P$ and ${ }_{2} P$ denote 1 ates of production and ${ }_{1} L$ and ${ }_{2} L$ denote rates of loss These production/loss rates are given by

$$
\begin{aligned}
& { }_{1} P=2\left(k_{3}\right)\left({ }_{3} u\right)+\left(k_{4}\right)\left({ }_{2} u\right) \\
& { }_{2} P=\left(k_{1}\right)\left({ }_{3} u\right)\left({ }_{1} u\right) \\
& { }_{1} L=\left(k_{1}\right)\left({ }_{3} u\right)+\left(k_{2}\right)\left({ }_{2} u\right) \\
& { }_{2} L=\left(k_{2}\right)\left({ }_{1} u\right)+k_{4}
\end{aligned}
$$


The constant values are $3^{u}=37 \times 10^{16} \mathrm{~mol}, k_{1}=163 \times 10^{-16} \mathrm{~mol}^{-2} \mathrm{~s}^{-1}$, and $k_{2}=$ $466 \times 10^{-16} \mathrm{~mol}^{-2} \mathrm{~s}^{-1}$ The time varying rate coefficient functions (which model chemical leactions driven by the absorption of sunlight) are given by

$$
k_{j}(t)=\left\{\begin{array}{ll}
\exp \left(\frac{a_{j}}{\cos (-\omega t)}\right) & \text { if } \cos (-\omega t)<0 \\
0 & \text { otherwise }
\end{array}, \quad j=3,4\right.
$$

with $a_{3}=2262, a_{4}=7601, \omega=2 \pi /(86,400 \mathrm{~s})$, and the $k_{j}$ are in units of mol $^{-1} \mathrm{~s}^{-1}$ Note that the constant $86,400 \mathrm{~s}$ is one day, so that the time varying reaction rate coefficient functions $\left(k_{3}\right.$ and $\left.k_{4}\right)$ have $24 \mathrm{~h}$ period $T$ 


\section{Mathematics Background}

\section{C.1 Power Series}

A power series is a series of the form

$$
c_{0}+c_{1}(x-a)+c_{2}(x-a)^{2}+\quad+c_{n}(x-a)^{n}+,
$$

in which $a$ and the $c_{i}, i=0,1,2$, are constants If a particular value is given to $x$, we than obtain an infinite series of numbers The special case $a=0$ occurs frequently, in which case the series becomes

$$
c_{0}+c_{1} x+c_{2} x^{2}+c_{3} x^{3}+\quad+c_{n} x^{n}+
$$

Most often, we use the $\Sigma$-notation, writing

$$
\sum_{n=0}^{\infty} c_{n}(x-a)^{n} \quad \text { and } \quad \sum_{n=0}^{\infty} c_{n} x^{n}
$$

It a power series converges for certain values of $x$, wo may define a function of $x$ by setting

$$
f(x)=\sum_{n=0}^{\infty} c_{n}(x-a)^{n} \quad \text { ol } \quad g(x)=\sum_{n=0}^{\infty} c_{n} x^{n}
$$

for those values of $x$ The majolity of functions which are encountered in the principal problems of mathematics and its applications to science and technology can be represented by convergent power series (with certain exceptions for the value a) [35] $A$ function that can be locally represented by a power series is called an analytuc function The class of analytic functions is closed with respect to the fundamental operations of arithmetic, algebra and analysis

\section{11 Taylou's Series}

A power series of the form

$$
f(x)=\sum_{n=0}^{\infty} \frac{f^{(n)}(a)}{n !}(x-a)^{n}
$$

is called the Taylor series for $f$ about the point a on the expansion of $f$ into a power sertes about $a[35]$

\section{C.1.2 Maclaun in Series}

For the special case $a=0$, the Taylor selies (144) is

$$
f(x)=\sum_{n=0}^{\infty} \frac{f^{(n)}(0)}{n !} x^{n}
$$

The right side of (145) is called the Maclaur $n$ serzes for $f$ [35]

\section{1.3 Taylor's Theorem with Remainder}

Suppose that $f, f^{\prime}, f^{(2)}, \quad, f^{(n)}, f^{(n+1)}$ are all continuous on some inter val containing $a$ and $b$ Then there is a number $\xi$ between $a$ and $b$ such that

$$
f(b)=f(a)+\frac{f^{\prime}(a)}{1 !}(b-a)+\frac{f^{(2)}(a)}{2 !}(b-a)^{2}+
$$




$$
+\frac{f^{(n)}(a)}{n !}(b-a)^{n}+\frac{f^{(n+1)}(\xi)(b-a)^{n+1}}{(n+1) !},
$$

i e, the remainder $R_{n}$ is given by the formula

$$
R_{n}=\frac{f^{(n+1)}(\xi)(b-a)^{n+1}}{(n+1) !}
$$

\section{C.2 Linear Systems}

Central to the study of linear algebra is the algebiaic system known as a vector space $\mathrm{V}$ over a field $\mathbf{F}$ Although much of what follows applies to general vector spaces, for our purposes we shall need only the concept of the $n$-dimensional real vector space $\mathcal{R}^{n}$ over the field of real numbers $\mathcal{R}$, with which most computer scientists are alıeady familiar

A general linear system of $m$ linear equations in $n$ unknowns $x_{1}, \quad, x_{n}$ has the form

$$
\begin{array}{ll}
E_{1} & a_{11} x_{1}+a_{12} x_{2}+a_{13} x_{3}+a_{22}+a_{1 n} x_{n}=b_{1} \\
E_{2} & a_{21} x_{1}+a_{23} x_{3}+a_{2 n} x_{n}=b_{2} \\
E_{3} & a_{31} x_{1}+a_{32} x_{2}+a_{33} x_{3}+a_{3 n} x_{n}=b_{3} \\
& \\
E_{m} & a_{m 1} x_{1}+a_{m 2} x_{2}+a_{m 3} x_{3}+a_{m n} x_{n}=b_{m},
\end{array}
$$

where the coefficients $a_{i j}$ are real numbers and the constants $b_{i}$ are also in $R$ Oul problem is to determine all points $\left(x_{1}, x_{2}, x_{3}, \quad, x_{n}\right)^{\Gamma} \in \mathcal{R}^{n}$ whose components satisfy all the equations in system (148)

System (148) can be expressed more efficiently in terms of matrix multiplication as

$$
\left(\begin{array}{llll}
a_{11} & a_{12} & a_{13} & a_{1 n} \\
a_{21} & a_{22} & a_{23} & a_{2 n} \\
a_{31} & a_{32} & a_{33} & a_{3 n} \\
& & & \\
a_{m 1} & a_{m 2} & a_{m 3} & a_{m n}
\end{array}\right)\left(\begin{array}{c}
x_{1} \\
x_{2} \\
x_{3} \\
\\
x_{n}
\end{array}\right)=\left(\begin{array}{c}
b_{1} \\
b_{2} \\
b_{3} \\
\\
b_{m}
\end{array}\right)
$$

If we use matix multiplication to compute the $i$ th entry in this column-vector equation (149), wc obtain the ith equation in system (148)

l.et us use the notations $\hat{x}, \hat{b}$, etc, to denote both $n$-dimensional $10 \mathrm{w}$ vectors and $n$ dimensional column vectors in $\mathcal{R}^{n}$, depending on the context $\mathrm{E} g$ equation (149) may be written

$$
A \hat{x}=\hat{b},
$$

where the $m \times n$ matrix $A=\left(a_{i j}\right)$ is the coefficient matrix,

$$
\hat{x}=\left(\begin{array}{c}
x_{1} \\
x_{2} \\
x_{3} \\
\\
x_{n}
\end{array}\right)
$$

is the column vector of unknowns, and

$$
\hat{b}=\left(\begin{array}{c}
b_{1} \\
b_{2} \\
b_{3} \\
\\
b_{m}
\end{array}\right)
$$


is the column vector of constants For our purposes, we shall say that the matiix $A$ maps or transforms the vector $\ddot{x}$ to the vector $\hat{b}$ We now face the problem of finding all column vectors $x$ such that (150) holds [36]

\section{C.2.1 Matrices}

To simplify computations, the matrix $A$ in $(150)$ can be dealt with instead of the equations in (148) This gives tise to matrix methods Although these matrix methods can be accomplished in principle, the calculation in actual practice is often so computationally intensive as to render the problem impractical or altogether impossible An understanding of the properties of some special matrices is therefore useful in selecting or massaging a numerical method Several types of matrices, operations and principles are described briefly here, without proof

An $m \times n$ matrix (pluial matrices) is a rectangulas array of elements with $m$ lows and $n$ columns in which not only is the value of an element important, but also its position in the array $\mathrm{Eg} A$ in $(149,150)$

A $1 \times n$ matrix $\hat{x}=\left(x_{1}, x_{2}, \quad, x_{n}\right)$ is called an $n$-dimensional row vector An $n \times 1$ matrix $\hat{x}=\left(x_{1}, x_{2}, \quad, x_{n}\right)^{T}$ is called an $n$-dimensional column vector $\mathrm{Eg} \quad \hat{x}$ in $(149-151)$

Let, $A$ be an $m \times n$ matix and $B$ an $n \times r$ matiix The matnix product, or malrix-matrix product of $A$ and $B$, denoted $A B$, is an $m \times 1$ matrix $C$ whose entries $c_{i j}$ ale given by

$$
c_{i j}=\sum_{k=1}^{n} a_{i k} b_{k j}, \quad \forall i=1,2, \quad, m, \quad \text { and } \quad \forall j=1,2, \quad, r
$$

Let $A$ be an $m \times n$ matrix and $\hat{x}$ be an $n$-dimensional vector The matrax-vector product of $A$ and $\hat{x}$, denoted $A \hat{x}$, is an m-dimensional columu vector $\hat{b}$ whose entries $b_{i}$ are given by

$$
b_{i}=\sum_{k=1}^{n} a_{i k} x_{k}, \quad \forall i=1,2, \quad, m
$$

E g the product $A \hat{x}$ in $(149,150)$

A square matrix has the same number of lows as columns

$\Lambda$ diagonal matrix is a square matrix $D=\left(d_{i j}\right)$ with $d_{i j}=0$ whenever $i \neq j \mathrm{Eg}$

$$
A=\left(\begin{array}{rrr}
10 & 0 & 0 \\
0 & 30 & 0 \\
0 & 0 & 20
\end{array}\right)
$$

The adentuty matrix of order $n, I_{n}=\left(\delta_{i j}\right)$, is the diagonal matrix with entries

$$
\delta_{i j}= \begin{cases}1 & \text { if } i=j \\ 0 & \text { if } i \neq j\end{cases}
$$

When the size of $I_{n}$ is cleal, this matrix is sometimes simply written as $I$

An upper-triangular $n \times n$ matrix $U=\left(u_{i j}\right)$ has entries $u_{i j}=0, \forall i>j$, i e the elements below the main diagonal are zero We will call a matrix structly upper-triangular if it is an upper-triangular matrix in which the diagonal elements are zeı, i $e$ the elements on and below the main diagonal are all zero Similatly, a lower-triangular $n \times n$ matix $L=\left(l_{i j}\right)$ has entries $l_{\imath j}=0, \forall i<j$, ie the elements ahove the main diagonal are zero We will call a matrix striclly lower-triangular if it is a lower-triangular matrix in which the diagonal elements are zero, $\mathrm{i}$ e the elements on and above the main diagonal are all zero

If $\exists$ an $n \times n$ matrix $A^{-1} \ni A A^{-1}=4^{-1} A=I$, theu the matrix $A^{-1}$ is called the inverse of $A$ It can be shown that to calculate the inverse of a general $n \times n$ matrix requires $O\left(n^{3}\right)$ multiplications/divisions and additions/subtractions For the special case of 
a diagonal matrix $A$, however, the inverse $A^{-1}$ is obtained by simply inverting the diagonal elements, and therefore requires only $n$ divisions $\mathrm{Eg}$ the inverse of $A$ in equation (155) is

$$
A^{-1}=\left(\begin{array}{ccc}
\frac{1}{10} & 0 & 0 \\
0 & \frac{1}{30} & 0 \\
0 & 0 & \frac{1}{20}
\end{array}\right)
$$

The transpose of an $m \times n$ matrix $A=\left(a_{i j}\right)$ is the $n \times m$ matrix $A^{T}=\left(a_{j i}\right)$

A square matrix $A$ is said to be symmetric if $A=A^{T}$

The determinant of an $n \times n$ matrix $A$, $\operatorname{denoted} \operatorname{det} A$ or $|A|$, is defined recursively as tollows

1 If $A=(a)$ is a $1 \times 1$ matrix, then $\operatorname{det} A=a$

2 If $A$ is an $n \times n$ matrix, with $n>1$, then the minor $M_{i j}$ is the determinant of the $(n-1) \times(n-1)$ submatrix of $A$ obtained by deleting the $i$ th row and $j$ th column of the matrix $A$, and the determinant of $A$ is given either by

$$
\operatorname{det} A=\sum_{j=1}^{n}(-1)^{i+j} a_{i j} M_{i j}, \quad \text { for any } i=1,2, \quad, n,
$$

ol

$$
\operatorname{det} A=\sum_{i=1}^{n}(-1)^{i+j} a_{i j} M_{i j}, \quad \text { for any } j=1,2, \quad, n
$$

E g the determinant of the matiix

$$
A=\left(\begin{array}{rrr}
10 & 5 & 0 \\
2 & 30 & -4 \\
0 & 3 & 20
\end{array}\right)
$$

is 5920 It can be shown that to calculate the determinant of a general $n \times n$ mati ix requires $O(n !)$ multiplic ations/divisions and additions/subtractions Even tor relatively small values of $n$, the number of calculations becomes unwieldy The calculation of the delerminant of a diagonal matrix, however, requires only $n$ multiplications and is simply the product of the diagonal elements $E g$ the determinant of $A$ in equation (155) is 6000

If the linear system $A \hat{x}=\hat{b}$ has a unique solution for each $m$-dimensional column vector $\hat{b}$, then we say that the matrix $A$ is non-singular This is equivalent to saying that $A \hat{x}=\hat{0}$ has the unique solution $\hat{x}=\hat{0}$, or to say that the inverse $A^{-1}$ exists, or to say that $\operatorname{det} A \neq 0$ Other wise the matrix $A$ is singular

The $n \times n$ matrix $A$ is said to be Strictly Diagonally Dominant (SDD) when

$$
\left|a_{i i}\right|>\sum_{\substack{j=1 \\ j \neq i}}^{n}\left|a_{i j}\right|, \quad \forall i=1,2, \quad, n
$$

$A$ matrix $A$ is Posttive Definite $(P D)$ if it is symmetric and if $\hat{x}^{T} A \hat{x}>0$ for every $n$ dimensional column vector $\ddot{x} \neq \hat{0}$

An $n \times n$ matrix $A$ is called a band matrix or banded matrax if $\exists$ integers $p, q, \ni 1<p$, $q<n$, and $a_{i j}=0$ whenever $i+p \leq j$, or $j+q \leq i$ The bandwidth of a band matrix is defined to be $w=p+q-1 \mathrm{Eg}$ the matrix $A$ in equation (160) is a band matrix with $p=q=2$ and bandwidth $w=3$

The definition of band nutrices forces those matices to concentrate. all their nonzero entiles about the diagonal Two special cases of band matrices that occul often in practice 
have $p=q=2$ and $p=q=4$ The matrix of bandwidth 3 , occuning when $p=q=2$, is encountered in connection with the study of cubic spline approximations These matrices are called tridiagonal, since they have (loosely speaking) a main diagonal of width 3 The matrix $A$ in equation (160) above is tridiagonal is

The norm $\|\hat{x}\|$ of a $n$-dimensional vector $\ddot{x}$ can be defined in several ways, one of which

$$
\|\hat{x}\|=\sqrt{x_{1}^{2}+x_{2}^{2}+}+x_{n}^{2},
$$

which is called the $l_{2}$ norm of $\ddot{x} \quad[37]$

\section{22 Eigenvalue Problem}

Let

$$
A \hat{x}=\dot{b}
$$

represent an $n \times n$ linear system $A$ nonzero vector $x$ is called an eigenvector or characteristic vector of $A$ if there exists a scalar $\lambda$ such that

$$
A \ddot{x}=\lambda \hat{x},
$$

i e $A$ maps $\hat{x}$ into a scalar multiple of itself The scalar $\lambda$ is called the ergenvalue or chaructenstuc malue of $A$ corresponding to $x$ [38] The set of eigenvalues is called the spectrum of $A$ The largest of the absolute values of the eigenvalues of $A$ is ralled the spectral radius of $A$, denoted $\rho(A)$

The problem of determining the cigenvalues and cigenvectors of a matilix is called an eigenvalue problem Problems of this type occur in connection with physical and technical applications [29]

\section{C.3 Lipschitz Condition}

A function $\hat{\phi}(y)$ is said to satisfy a Lipschatz conditzon in a neighborhood $N(\grave{y})$ of $y$ if $\forall \dot{y}^{*} \in N(\ddot{y})$,

$$
\left\|\dot{\phi}(y)-\grave{\phi}\left(\dot{y}^{*}\right)\right\| \leq L\left\|\dot{y}-\hat{y}^{*}\right\|
$$

where $L$ is called the Lipschitz constant This condition essentially says that the magnitude of the slope of the line through $(\ddot{y}, \dot{\phi}(\ddot{y}))$ and $\left(\hat{y}^{*}, \dot{\phi}\left(\dot{y}^{*}\right)\right)$ is bounded everywhere in the neighborhood [14]

\section{C.4 Differential Equations}

An equation involving one dependent variable and its derivatives with respect to one on more independent variables is called a differential equatıon (DE) Many of the general laws of nature - in physics, chemistry, biology, and astronomy - find their most natural expression in the language of differential equations Applications also abound in mathematics itself, especially in geometry, and in engineering, economis $s$, and many other fields of applied science

It is easy to understand the reason behind this broad utility of differential equations The reades will recall that if $y=f(x)$ is a given function, then its derivative $d y / d x$ can be interpreted as the rate of change of $y$ with 1espect to $x$ In any natural process, the variables involved and their rates of change are connected with one another by means of the basic scientific principles that goven the process When this connection is expressed in mathematical symbols, the result is often a differential equation

The following example may illuminate these remalks Accolding to Newton's second law of motion, the acceleration $a$ of a body of mass $m$ is proportional to the total force $F$ acting on it, with $1 / m$ as the constant of proportionality, so that $a=F^{1} / m$ or

$$
m a=H^{2}
$$


Suppose, for instance, that a body of mass $m$ falls freely under the influence of gravity alone In this case the only force acting on it is $m g$, where $g$ is the acceleration due to gravity If $y$ is the distance down to the body from some fixed height, then its acceleration is $d^{2} y / d t^{2}$, and (166) becomes

$$
m \frac{d^{2} y}{d t^{2}}=m g
$$

or

$$
\frac{d^{2} y}{d t^{2}}=g
$$

If we alter the situation by assuming that air exerts a resisting force proportional to the velocity, then the total force acting on the body is $m g-k(d y / d t)$, and $(166)$ becomes

$$
m \frac{d^{2} y}{d t^{2}}=m g-k \frac{d y}{d t}
$$

Equations (168) and (169) are the differential equations that express the essential at tributes of the physical processes under consideration [39]

Various analytical methods exist for solving I)Es We shall not examine them here, since we are interested in numerical methods

\section{4.1 Ordinary Differ ential Equations}

As further examples of differential equations, we list the following

$$
\begin{gathered}
\frac{d y}{d t}=-k y, \\
m \frac{d^{2} y}{d t^{2}}=-k y, \\
\frac{d y}{d x}+2 x y=e^{-x^{2}}, \\
\frac{d^{2} y}{d x^{2}}-5 \frac{d y}{d x}+6 y=0, \\
\left(1-x^{2}\right) \frac{d^{2} y}{d x^{2}}-2 x \frac{d y}{d x}+p(p+1) y=0, \\
x^{2} \frac{u^{2} y}{d x^{2}}+x \frac{d y}{d x}+\left(x^{2}-p^{2}\right) y=0
\end{gathered}
$$

The dependent variable in each of these equations is $y$, and the independent variable is either $t$ or 2 The letters $k, m$, and $p$ repiesent constants An Ordinary Differentual Fquation $(O D E)$ is one in which there is only one independent variable, so that all the derivatives occuring in it are ordinaly derivatives Each of these equations is ordinary the order of a differential equation is the order of the highest derivative present Equations (170) and (172) are first-order equations, and the others are second-order Equations (174) and (175) are classic al, and ate called Legendre's equation and Bessel's equation, respectively Fach has a vast literature and a history rearhing bark hundıeds of years [39]

The most important type of differential equation is the linear equation, in which the derivative of highest order is a linear function of the lower order derivatives Thus the general fiıst-order linear equation is

$$
\frac{d y}{d x}=q(x) y+\imath(x)
$$

the gener al second-order linear equation is

$$
\frac{d^{2} y}{d x^{2}}=\eta(x) \frac{d y}{d x}+q(x) y+\imath(x)
$$


and so on It is understood that the coefficients on the right in these expressions, namely, $p(x), q(x), r(x)$, etc, are functions of $x$ alone [39] If $r(x)=0$ in (176) or (177) then the ODE is homogeneous Otherwise it is znhomogeneous [29] The general solution of (176) is

$$
y=e^{\int q(x) d x}\left(\int \imath(x) e^{-\int q(x) d x} d x+c\right)
$$

The general ODE of the $n$ th-order is

$$
F\left(x, y, \frac{d y}{d x}, \frac{d^{2} y}{d x^{2}}, \quad, \frac{d^{n} y}{d x^{n}}\right)=0,
$$

or, using the prime notation for derivatives,

$$
F\left(x, y, y^{\prime}, y^{\prime \prime}, \quad, y^{(n)}\right)=0
$$

It is normally a simple task to venify that a given function $y=y(x)$ is a solution of an equation like (179) All that is necessary is to compute the derivatives of $y(x)$ and to show that $y(x)$ and these delivatives, when substituted into the equation, reduce it to an identity in $x$ In this way we see that

$$
y=e^{2 x} \quad \text { and } \quad y=e^{3 x}
$$

are both solutions of the second-order equation

$$
y^{\prime \prime}-5 y^{\prime}+6 y=0,
$$

and, more generally, that

$$
y=c_{1} e^{2 x}+c_{2} e^{3 x}
$$

is also a solution for every choice of the constants $c_{1}$ and $c_{2}$ [39] Equation (183) is caller the general solution to (182)

In many engineering applications we are not interested in the general solution of a given differential equation but only in the particular solution $y(x)$ satisfying a given initual conditron (IC), say, the condition that at some point $x_{0}$ the solution $y(x)$ has a prescribed value. $y_{0}$, briefly

$$
y\left(x_{0}\right)=y_{0}
$$

A fisst-order differential equation together with an initial condition is called an Initral Value Problem (IVP) To solve such a problem we must find the particulas solution of the equation which satisfies the given initial condition $\mathrm{Eg}$ the IVP

$$
\left(x^{2}+1\right) y^{\prime}+y^{2}+1=0, \quad y(0)=1
$$

has the particular solution [29]

$$
y=\frac{1-x}{1+x}
$$

As another example, the IVP

$$
\ddot{x}+8 \dot{x}+25 x=0, \quad x(0)=x_{0}, \quad \dot{x}(0)=0,
$$

has the particular solution

$$
x(t)=x_{0}\left(\cos 3 t+\frac{4}{3} \sin 3 t\right) e^{-4 t}
$$

Notice that IVP (185) is first-order with one IC and that IVP (187) is second-older with two IC's This is no arcident A solution of a differential equation of the order $n$ (lineas 
or not) is called a general solution if it contains $n$ arbitrary independent constants Here independence means that the solution cannot be reduced to a form containing less than $n$ arbitiary constants If we assign definite values to the $n$ constants, then the solution so obtained is called a particular solution of that equation [29]

Let us now seek a nontrivial solution $y(x)$ of the equation

$$
y^{\prime \prime}+\lambda y=0
$$

that satisfies the boundary conditions (BCs)

$$
y(0)=0 \quad \text { and } \quad y(\pi)=0
$$

The parameter $\lambda$ in (189) is free to assume any real value whatevel, and part of ont task is to discover the $\lambda$ 's for which the problem can be solved In our previous problems we have considered only mitral value problems, in which the solution of a second-onder equation is sought that satisfies two conditions at a single value of the independent variable Here we have an entirely different situation, for we wish to satisty one condition at each of two distinct values of $x$ Problems of this kind are called boundary value problems ( $B V P s$ ), and in general they are more difficult and far reaching - in both theny and practice - than initial value problems Although we will not pursue the theory hehind BVPs like equation (189) here, its non-trivial solutions are

$$
y(x)=c_{1} \sin \sqrt{\lambda} x,
$$

with $\lambda=n^{2}, n=1,2,3$,

\section{C.42 Systems of Ordinary Differential Equations}

Sometimes we shall be concened with a first-order system of ordinary differential equations of the form

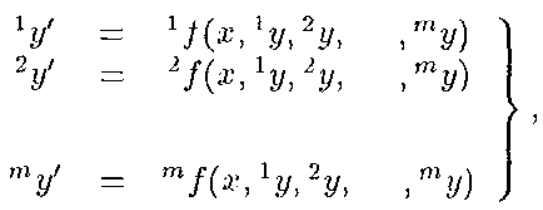

where

$$
{ }^{j} y^{\prime}=\frac{d}{d x} j y(x)
$$

We immediately abhıeviate the phuase italicized above to 'first-order system', or just 'system' The system (192) can be written in vector form as

$$
\ddot{y}^{\prime}=\hat{f}(x, y),
$$

where $\ddot{y}=\left({ }^{1} y,{ }^{2} y, \quad,{ }^{m} y\right)^{T}$ and $\hat{f}=\left({ }^{1} f,{ }^{2} f, \quad,{ }^{m} f\right)^{T}$, so that $\hat{f} \mathcal{R} \times \mathcal{R}^{m} \rightarrow \mathcal{R}^{m}$

We note that each ${ }^{j} f$ depends on ${ }^{1} y,{ }^{2} y,{ }^{m} y$, i e , the system is coupled Were it the case that each ${ }^{i} f$ depended on ${ }^{j} f$ alone, the system would be uncoupled, and each equation in it could be handled independently of the rest It is this coupling that is the essence of a system, an uncoupled system is not essentially different from a scalar DE

The general solution of a first-order system of dimension $m$ contains, in general, $m$ arbitrary constants, thus, e $g$, it is easily checked by substitution that the 2-D system

$$
\left.\begin{array}{rl}
{ }^{1} y^{\prime} & ={ }^{1} y / x+{ }^{2} y x \\
{ }^{2} y^{\prime} & =x\left[\left({ }^{2} y\right)^{2}-1\right] /{ }^{1} y
\end{array}\right\}
$$

is satisfied by

$$
\begin{aligned}
& { }^{1} y(x)=x\left[\cos \left(C_{1} x+C_{2}\right)\right] / C_{1} \\
& { }^{2} y(x)=-\sin \left(C_{1} x+\left(C_{2}\right)\right.
\end{aligned}
$$


for any values (with the exception of $C_{1}=0$ ) of the arbitrary constants $C_{1}^{1}, C_{2}$ For the general $m$-dimensional system, the $m$ arbitrary constants can be fixed by imposing $m$ initial conditions If these $m$ conditions take the form of demanding that the ${ }^{j} y, j=1,2, \quad, m$ all take given values at the same initial point, then the system together with the conditions constitute an IVP Wiiting the system in the vector form (194), the general IVP thus takes the folm

$$
\ddot{y}^{\prime}=\hat{f}(x, \hat{y}), \quad \ddot{y}(a)=\hat{\eta}, \quad \hat{f} \mathcal{R} \times \mathcal{R}^{m} \rightarrow \mathcal{R}^{m}
$$

where

$$
\hat{\eta}=\left({ }^{1} \eta,{ }^{2} \eta, \quad,{ }^{m} \eta\right)^{T}
$$

Once again, we abbreviate the nomenclature, and henceforth refer to (197) as a 'problem'

If in (197) $f$ is independent of $x$, the problem (and the systen it involves) is said to be autonomous, and to be non-autonomous otherwise It is always possible, at the cost of raising the dimension by 1 , to write a non-autonomous problem in autonomous form All one need do is add an extra scalar equation ${ }^{m+1} y^{\prime}=1$ with IC ${ }^{m+1} y(a)=a$, which implies that ${ }^{m+1} y \equiv x$, so that the new $(m+1)$-dimensional system is cleally autonomous $\mathrm{E} g$, if we add to $(195)$ the ICs ${ }^{1} y(1)=1,{ }^{2} y(1)=0$, the resulting 2 -D non-autonomous problem can be rewritten as

$$
\begin{array}{ll}
{ }^{1} y^{\prime}={ }^{1} y / /^{3} y+{ }^{2} y^{3} y & { }^{1} y(1)=1 \\
{ }^{2} y^{\prime}={ }^{3} y\left[\left({ }^{2} y\right)^{2}-1\right] / /^{1} y & { }^{2} y(1)=0 \\
{ }^{3} y^{\prime}=1 & { }^{3} y(1)=1,
\end{array}
$$

a 3-D autonomous problem, with solution

$$
\begin{aligned}
& { }^{1} y(x)=x \cos (x-1) \\
& { }^{2} y(x)=-\sin (x-1) \\
& { }^{3} y(x)=x
\end{aligned}
$$

Since we regand the dimension of the problem as being anbitrary, there is clearly no loss of generality in assuming that the general $m$-dimensional problem is autonomous [14]

\section{43 Partial Differential Equations}

Physical systems in many fields of applied science and engineening can be described in terms of mathematical systems composed of equations containing partial derivatives A partial differential equation (PDE) is an equation involving several independent variables $x, y, z$, a function $u$ of these variables, and the partial derivatives of the dependent function $u$ with respect to the independent variables 'These variables nuay be time and one or several coordinates in space Symbolically, the general PDE is

$$
F\left(x, y, z, \quad, u, u_{x}, u_{y}, u_{z}, \quad, u_{x x}, u_{y y}, \quad, u_{x y}, u_{x z}, \quad\right)=0
$$

The order of the highest derivative is called the onder of the equation Just as in the case of ODEs, we say that a PDE is linear if it is of the finst degree in the dependent variable and its partial derivatives [19]

Sorne important linear PDEs of the second-order are

$$
\begin{aligned}
& \frac{\partial^{2} u}{\partial t^{2}}=c^{2} \frac{\partial^{2} u}{\partial x^{2}} \\
& \frac{\partial u}{\partial t}=c^{2} \frac{\partial^{2} u}{\partial x^{2}} \\
& \frac{\partial^{2} u}{\partial x^{2}}+\frac{\partial^{2} u}{\partial y^{2}}=0 \\
& \frac{\partial^{2} u}{\partial x^{2}}+\frac{\partial^{2} u}{\partial y^{2}}=f(x, y)
\end{aligned}
$$




$$
\frac{\partial^{2} u}{\partial x^{2}}+\frac{\partial^{2} u}{\partial y^{2}}+\frac{\partial^{2} u}{\partial z^{2}}=0
$$

Equations (202-206) are called the 1-D wave equation, 1-D heat equation, 2-D Isaplace cquation, 2-D Poisson equation, and 3-D Laplace equation, respectively Here $c$ is a constant, $t$ is time, and $x, y, z$ are orthogonal space coordinates [29] Each of the above equations is profoundly significant in theor etical physics, and their study has stimulated the development of many important mathematical ideas In general, PDEs ar ise in the physics of continuous media - in problems involving electric fields, fluid dynamics, diffusion, and wave motion Their theory is very different from that of ODEs, and is much more difficult in almost every respect [39]

A solution of a PDE in some region $R$ of the space of the independent valiables is a function which has all the partial derivatives appearing in the equation in some domain containing $\mathcal{R}$, and satisfies the equation everywhere in $\mathcal{R}$ (One often merely requires that the function is continuous at the boundary of $\mathcal{R}$, has those derivatives in the interior of $R$, and satisfies the equation in the interior of $\mathcal{R}$ [29]) $\mathrm{E} g$ if

$$
u_{x x}-u_{t t}=0 \text {, }
$$

which is equation (202) with $c=1$, then

$$
\begin{aligned}
& u_{1}(x, t)=\sin \pi x \cos \pi t \\
& u_{2}(x, t)=\sin 2 \pi x \cos 2 \pi t \\
& u_{n}(x, t)=\sin n \pi x \cos n \pi t
\end{aligned}
$$

are all solutions, as is easily seen by direct substitution The result (208) reveals an inuportant characteristic of PDEs that is rommon to ODEs, too a single equation, like (207), for one. function $u$ may possess many particular solutions Thus one concern in any problem is to develop certain auxuliary conditions that serve to isolate or chararterize an individual solution for a given problem [19]

In general, the totality of solutions of a PDE is very large $\mathrm{Eg}$, the functions

$$
u=x^{2}-y^{2}, \quad u=e^{x} \cos y, \quad u=\ln \left(x^{2}+y^{2}\right),
$$

which are entirely different from each other, are solutions of (204), as one nuay easily verify The unique solution of a PDF, cor esponding to a given physical problem will be obtained by the use of additional information arising from the physical situation $\mathrm{E} g$, in some cases the values of the required solution of the problem on the boundary of some donain, boundary conditions (BCS) will be given, in other cases when the tinne $t$ is one of the variables, the values of the solution at $t=0$, inttal condztrons (ICs) will be prescribed [29]

Certain types of boundary conditions appear and are appropriate in the mathentatical models of initial-boundary-value-problems (IBVPs) These common conditions are classified as boundary conditions of

1 the first type, or the Drrichlet condition the dependent variable is prescribed along the boundary

2 the second type, or the Neumann condition the nom mal derivative of the dependent variable is prescribed along the boundary

3 the thind type a relation between the dependent variable and its normal derivative is prescribed along the boundary [19]

Sperial cases of the 2-D second-order equation

$$
a \frac{\partial^{2} u}{\partial x^{2}}+b \frac{\partial^{2} u}{\partial x \partial y}+c \frac{\partial^{2} u}{\partial y^{2}}+d \frac{\partial u}{\partial x}+e \frac{\partial u}{\partial y}+f u+y=0,
$$


where $a, b, c, d, e, f$, and $g$ may be functions of the independent variables $x$ and $y$ and of the dependent variable $u$, occur more frequently than any other because they are often the mathematical form of one of the conservation principles of physics Equation (210) is said to be elliptic when $b^{2}-4 a c<0$, parabolic when $b^{2}-4 a c=0$, and hyperbolic when $b^{2}-4 a c>0$ These are called canonical forms of (210) [40]

As an impoltant application, let us consider the temperature in a long thin bal or wire of constant cross section and homogenenus material which is oriented along the $x$-axis and is perfertly insulated laterally, so that heat flows in the $x$-direction only Then $u$ depends only on $x$ and time $t$, and the heat equation (96) becomes the 1-D heat equation (203), with $c^{2}=k$ Let us examine the case when the ends $x=0$ and $x=1$, of the bas are kept at temperatule zero Then the Dirichlet BCs ase

$$
u(0, t)=u(L, t)=0 \quad \forall t
$$

Let the initial temperature distribution in the bar be

$$
u(x, 0)=\sin \left(\frac{\pi x}{L}\right)
$$

The individual solution $u(x, t)$ of this linear, second-order, 1-D, parabolic IBVP $(203,211$, $212)$ is $[29]$

$$
u(x, t)=\sin \left(\frac{\pi x}{L}\right) e^{-(c \pi / L)^{2} t}
$$




\section{Numerical Methods}

\section{D.1 Introduction}

The ultimate aim of the field of numerical analysis is to provide convenient methods for obtaining useful solutions to mathematical problems and for extracting useful information from available solutions which are not expressed in tractable forms Such problems may each be formulated, e g, in terms of an algebraic or transcendental equation, an ODE or PDE, or an integral equation, or in terms of a set of such equations [41] As a simple algebraic example, the problem of finding the zeroes of an $n$ th-degree real polynomial with $n \geq 5$ may have no analytical solution As a more complex PDE example the Navier-Stokes equations (85), for which there is no known analytical solution

This formulation may cor respond exactly to the situation which it is intended to describe, more often, it will not Analytical solutions, when available, may be precise in thenselves, but may be of unacceptable form because of the fact that they are not amenable to direct interpretation in numerical terms, in which case the numerical analyst may attempt to devise a method for effecting that interpıetation in a satisfactory way, or he may prefer to base his analysis instead upon the original formulation [41] As a simple algebraic example the problem of finding the zeroes of $p(x)=x^{2}-2$, whose exact analytical solution is cleally $x_{1}=-\sqrt{2}, x_{2}=+\sqrt{2}$, which are expressable in closed symbolic form but are not in a usable numerical form As a more complex example the integral formula for the solution of a first-ordeı linear ODE (178)

More frequently, there is no known method of obtaining the solution in a precise form, convenient or other wise In such a case, it is necessary either to attempt to approximate the problem satisfactoily by one which is amenable to precise analysis, to obtain an approximate solution to the oniginal problem by methods of numerical analysis, or to combine the two approaches

On the other hand, the problem itself may not be clearly defined, and the analyst nuay be provided only with its partial solution, perhaps in the form of a table of approximate data, together with a certain amount of information with regard to its reliability, or perhaps in terms of an integral defining a function which cannot be expressed in terms of a finite number of tabulated functions His purpose then is to obtain additional useful information concenning the function so described

Generally the numerical analyst does not strive for exactness Instead, he altempts to devise a method which will yield an approximation differing from exactness by less then a sperified tolerance, or by an amount which has less than a specified probability of exceeding that tolerance When the information supplied to him is inexact, he attempts both to obtain a dependable measure of the uncertainty which results from that inexactness and also to obtain an approximation which possesses a specified reliability compatible with that uncertainty

He tries to devise a procedure which would be capable of affording an arbitrarily high degice of accuracy, in a wide class of situations, if the reliability of given information and of available calculating devices were correspondingly high Even when successful in this attempt, he still seeks alternative procedures which may possess celtain advantages in convenience or efficiency in certain situations, but which may be of less general applicability, or which may have the property that the degree of accuracy obtainable, even under ideal circurnstances, cannot exceed a certain limit which depends upon the function to be aulalyzed In this last case, which is of fiequent occurrence, he attempts to ascertain that limit and to classify the situations in which it is not sufficiently high

Needless to say, there are relatively few situations in which all these objectives have heen ol can be, perfectly attained Howeve., research with these aims in view continues to provide rew procedures, as well as additional information with regard to the basic advantages and disadvantages of the older ones Additional impetus has been aftorded by the developinent 
of automatic desk calculators and, more iecently, of large-scale computers For example, certain methods had long been known to possess important theoretical advantages, but had not been convenient, from the point of view of the labor and time involved, for use in hand calculation or in calculation based on the use of the slide iule on of tables of logarithms, and hence had been considered as little more than mathematical curiosities However, technological developments have promoted several of them into a nuch more active status and have also created additional need for detailed reexamination and modification of other existing methods and for a search for new ones

One of the most rapidly expanding phases of numerical analysis is that which deals with the approximate solution of PDEs But a hasic understanding of the more involved problems which arise in that phase of the analysis depends stıongly upon familiarity with similar problems which arise, in a sonewhat simpler way, in connection with the solution of algebraic and tianscendental equations, the processes of interpolation and approxination, numerical difterentiation and integration, and the approximate solution of ODEs, in which only one independent variable is involved [41]

In the process of problem solving, it is possible to distinguish several move or less distinct phases The first phase is formulatıon In fommlating a mathematical model of a physical system, scientists should take into account beforehand the fact that they expect to solve a problem on a cornputer They will therefore provide for specific objectives, proper input data, adequate checks, and for the type and amount of output

Once a problem has been formulated, numerical methors, together with a preliminary error analysis, must be devised for solving the problem A nunerical method which can be used to solve a problem will be called an alyorithm An algoithm is a romplete and unambiguous set of proc edures leading to the solution of a mathematical problem The selection ol construction of appropriate algorithms propely falls within the scope of numercal analysis Having decided on a specific algorithm or set of algonithms for solving the problem, numerical analysts should consider all the sources of eiror that may affect the results They must consider how much accuracy is lequired, estimate the magnitude of the round-off and discretization ertors, determine an appropriate step size or the number of iter ations required, provide for adequate checks on the accurary, and make allowance for conective action in cases of nonconvergence

The third phase of problem solving is programming The progiammer must transform the suggested algorithn into a set of unambiguous step-by-step instu uctions to the computer [42]

\section{D.2 Euler's Method}

Although Euler's method is seldom used in practice, the simplicity of its derivation can be used to illustrate the techniques involved in the construction of some of the more advanced techniques, without the cumbersome algebia that accompanies these constructions

The object of the method is to obtain an approximation to the solution to the well-posed IVP

$$
\frac{d y}{d t}=f(y, t), \quad a \leq t \leq b, \quad y(a)=\alpha
$$

In actuality, a continuous approximation to the solution $y(l)$ will not be obtained, instead, approximations to $y$ will be generated at various values, called mesh points, in the interval $[a, b]$ Once the approximate solution is obtained at these points, the approximate solution at other points in the interval can be obtained by intelpolation The process of producing the approximate solntion to (214) at mesh points is often called mtegration of the IVP, since integration and differentiation are inverse processes $A$ particular solution to an IVP is often called a purticular integral

In this section, we niake the stipulation that the mesh points are equally distributed throughout the interval $[a, b]$ This condition is ensured by choosing a positive integer $N$ 
and selecting the mesh points $\left\{t_{0}, t_{1}, t_{2}, \quad, t_{N}\right\}$ where

$$
t_{i}=a+i h, \quad i=0,1,2, \quad, N
$$

The common distance, $h=(b-a) / N$, between successive points, is called the stepsize, or steplength A step between points of time is called a timestep (Mesh points are not always equally distributed however)

Suppose that $y(t)$, the unique solution to (214), has two continuous derivatives on $[a, b]$ Then we can represent $y(t)$ with a Taylor series and for each $i=0,1,2, \quad, N-1$,

$$
y\left(t_{i+1}\right)=y\left(t_{i}\right)+\left(t_{i+1}-t_{i}\right) y^{\prime}\left(t_{i}\right)+\frac{\left(t_{i+1}-t_{i}\right)^{2}}{2} y^{\prime \prime}\left(\xi_{i}\right)
$$

for sume number $\xi_{i}$ in $\left(t_{i}, t_{i+1}\right)$ If $h=t_{i+1}-t_{i}$, then

$$
y\left(t_{i+1}\right)=y\left(t_{i}\right)+h y^{\prime}\left(t_{i}\right)+\frac{h^{2}}{2} y^{\prime \prime}\left(\xi_{i}\right)
$$

and, since $y(t)$ satisfies the ODE (214),

$$
y\left(t_{i+1}\right)=y\left(l_{i}\right)+h f\left(y\left(t_{i}\right), t_{i}\right)+\frac{h^{2}}{2} y^{\prime \prime}\left(\xi_{i}\right)
$$

Euler's method constructs $y^{i} \approx y\left(t_{i}\right)$ for each $i=1,2, \quad, N$, by deleting the remaindes term Thus [37]

$$
\begin{aligned}
y^{\prime \prime} & =r, \\
y^{i+1} & =y^{i}+h f\left(y^{i}, t_{i}\right), \quad i=0,1, \quad, N-1
\end{aligned}
$$

Equation (219) is called the For ward Euler $(F E)$ method because the derivative $f\left(y^{i}, t_{i}\right)$ "looks for ward" to generatc $y^{i+1}$ In vector form, (219) becomes

$$
\begin{aligned}
\dot{y}^{0} & =\dot{\alpha}, \\
y^{i+1} & =\hat{y}^{i}+h \hat{f}\left(\dot{y}^{i}, t_{i}\right), \quad i=0,1, \quad, N-1
\end{aligned}
$$

$\Lambda$ sintilar Taylon appoximation, the Backward Euler (BE) methon is defined by

$$
\begin{aligned}
\dot{y}^{0} & =\dot{\alpha}, \\
\dot{y}^{i+1} & =\dot{y}^{i}+h \hat{f}\left(\dot{y}^{i+1}, t_{i+1}\right), \quad i=0,1, \quad, N-1
\end{aligned}
$$

Equation (221) is called the Backward Eulen method because the derivative $\hat{f}\left(\dot{y}^{i+1}, t_{i+1}\right)$ "looks backward" to generate $\hat{y}^{i+1}$

\section{D.3 Explicit Methods}

If the cullent mesh point $y^{n+k}$ is defined in terms of only the previous mesh points $y^{n+k-1}$, $\ddot{y}^{n+k-2}$, , then the method is explacit FE is explicit [37]

Advantages

- simple to implement

- often vely cfficient on parallel and vector computers

Disadvantages

- typically unstable for reasonably large stejsizc

- frequently requine prohibitively snall stepsizes and therefore require long exccution times 


\section{4 Implicit Methods}

If the method is not explicit, then the method is implacit BE is implicit [37]

Advantages

- typically has good stability characteristics

- often not constrained by stability to a small stepsize

Disadvantages

- much computational over head is usually necessary to solve the (potentially nonlinear) simultaneous algebraic system at each timestep

\section{D.5 One-Step Method}

A method which can hy written

$$
\ddot{y}^{n+1}=\ddot{y}^{n}+h \psi\left(\ddot{y}^{n}, t_{n}, h\right),
$$

where $h$ is given and the increment function $\dot{\psi}$ is determined by $\hat{f}$ and is a function of $\hat{y}^{n}$, $t_{n}$ and $h$ only, is called a one-step method [14] FE is a one-step method BE is implicitly one-step, i e although we may not be able to write the increment function $\hat{\psi}, \psi$ exists

\section{D.6 Local Truncation Error}

Since the object of numerical techniques is to determine sufficiently accurate approximations with minimal effort, we need a means for comparing the efficiency of various approximation methods The first device we consider is called the Loc al Truncation Error (LTE, $\hat{d}^{i+1}$ ), or local discretization error of the mothod The LTE at a specified step measures the locally produced anıount by which the exact solution to the problem fails to satisfy the method being used for the approximation [37]

If the approximation is calculated by

$$
\ddot{y}^{i+1}=\phi\left(\ddot{y}^{2}, h\right)
$$

then the L'T at $t_{i+1}$ is given by

$$
\hat{d}^{i+1}=\dot{\phi}\left(\grave{y}\left(t_{i}\right), h\right)-\grave{y}\left(t_{i+1}\right)
$$

The ten $\hat{\phi}\left(\hat{y}\left(t_{i}\right), h\right)$ in equation (224) is the value which the method would yield as an approximation to $\hat{y}(t)$ at $t_{i+1}$ by taking one step $h$ flom the actual value of $y(t)$ at $t_{i}$ The tern $\hat{y}\left(t_{i+1}\right)$ is the actual value of $\hat{y}(t)$ at $t_{i+1}$ Hence the LTE is in essence a measure of the amount by which the method deviates from the actual solution locally, due to discretization [14]

Let us consider as a test problem, the 1-D IVP

$$
\begin{aligned}
y & =-5 y, \\
y(0) & =1,
\end{aligned}
$$

whose actual solution is

$$
y(t)=e^{-5 t}
$$

Using FE with $h=0$ 1, we have

$$
\begin{aligned}
\phi\left(y^{i}, h\right) & =y^{i}+h f\left(y^{i}, t_{i}\right) \\
& =y^{i}+(01)\left(-5 y^{i}\right) \\
& =(05) y^{i},
\end{aligned}
$$


and

$$
\begin{aligned}
d^{i+1} & =\phi\left(y\left(t_{i}\right), h\right)-y\left(t_{i+1}\right) \\
& =(05) y\left(t_{i}\right)-y\left(t_{i+1}\right) \\
& =(05) e^{-5 t_{i}}-e^{-5 t_{i+1}}
\end{aligned}
$$

At $t_{10}=10$,

$$
\begin{aligned}
d^{10} & =(05) e^{(-5)(09)}-e^{(-5)(10)} \\
& \approx-00011834
\end{aligned}
$$

\section{D.7 Total Accumulated Error}

The next derice we consider is called the Total Accumulated Eiror (TAE'), or global truncatıon error 'The 'TAE at a specified step measures the total amount by which the exact solution to the problem fails to satisfy the method being used for the approximation The TAE at $t_{i}$ is given by [14]

$$
\hat{e}^{i}=\dot{y}^{i}-y\left(t_{i}\right)
$$

In the test problem, (225), using FE with $h=01$, at $t_{10}=10$, we have

$$
\begin{aligned}
e^{i} & =y^{i}-y\left(t_{i}\right) \\
& =(05)^{i} y^{0}-e^{(-5) t_{i}} \\
& =(05)^{10}(1)-e^{(-5)(10)} \\
& \approx-00057614
\end{aligned}
$$

\section{D.8 Order of Accuracy}

We frequently use the notation

$$
F(h)=O\left(h^{p}\right) \text { as } h \rightarrow 0,
$$

where $h$ is a scalar and $F$ may be either a scalar or a vector Notation (232) means that there exists a positive consiant $K$ such that $\left\|F^{\prime}(h)\right\| \leq K h^{p}$ for $h$ sufficiently close to zelo Nosmally, we do not bother to add the phrase 'as $h \rightarrow 0$ ' and merely write $F(h)=O\left(h^{p}\right)$, hut 'as $h \rightarrow 0$ ' is still jmplied This is of some importance, since $F(h)=O\left(h^{p}\right)$ is an asymptotus statement concerning what happens for sufficiently small $h$, its interpietation must not be dehased into implying that $F(h)$ is roughly the same size as $h^{p}$, legardless of the size of $h[14]$

If the LTE of a numesical method is $O\left(h^{p+1}\right)$, then we say that the method is order $p$ We commonly say, ' $F$ of $h$ is big $O$ of $h$ to the $p$ ', or ' $F$ of $h$ equals $O$ of $h$ to the $p$ ', or ' $F$ ' of $h$ is urder $h$ to the $p$ ', elc, or that 'a method is p-order accurate' $\mathrm{Eg} F$ 'E and BE are finst-order accurate, since the terms omitted from the Taylor series to derive these methods are

$$
F(h)=\frac{h^{2}}{2} y^{\prime \prime}\left(t_{i}\right)+\frac{h^{3}}{3 !} y^{\prime \prime \prime}\left(t_{i}\right)+
$$

and $F(h)$ is $O\left(h^{2}\right)$

A distinction should be made here between the use of $O\left(h^{p}\right)$ for small stepsizes $h$ in the analysis of numerical methrods, and the use of $O\left(h^{p}\right)$ for large data set size in the analysis of computer algorithms It the LTE of a numerical method is

$$
F(h)=u h^{3}+b h^{2}+c h
$$

$\mathrm{e} g$, then the LTE is $O(h)$, since the other terms of (234) are insignificant when $h \rightarrow 0$ and the method is zeloth-order accurate However, if a computer algorithm takes $F(h)$ time to 1 un, then the algoithm is $U\left(h^{3}\right)$, since the other terms of (234) are insignificant when $h \rightarrow \infty$ 


\section{D.9 Stability}

We are interested in using methods that will produce dependably accurate results for a wide range of problems One criterion we like to impose upon an algorithm whenever possible. is that small changes in the initial data produce correspondingly small changes in the final results An algorithm that satisfies this property is called stable, it is unstable when this criterion is not fulfilled Some algolithms will be stable for certain choices of initial data but not for all choices [37] Small changes in the initial data are called perturbatrons

Although definitions of many different kinds of stability are in use, we will use the following definition a method is said to be absolutely stable for a given step size $h$, if a perturbation $\hat{\epsilon}_{0}$ in the IC, results in a change no gxeater than $\hat{\epsilon}_{0}$ in the computed solution at all subsequent steps

E $\mathrm{g}$ we. will show that $\mathrm{FE}$ is stable for the test equation,

$$
y^{\prime}=\lambda \hat{y}, \quad \lambda \in \mathcal{C},
$$

which is a very specific ODE, used here in plare of an arbitrary problem We define the peiturbations

$$
\begin{array}{ll}
\hat{z}^{0}=\hat{y}^{0}+\hat{\epsilon}^{0} & \text { I(', } \\
\hat{z}^{k}=\hat{y}^{k}+\hat{\epsilon}^{k} & \text { for any step } \mathrm{k}
\end{array}
$$

We apply FE to $(235)$

$$
\begin{array}{ll}
\ddot{z}^{k+1}=\hat{z}^{k}+h \lambda \dot{z}^{k} & \text { perturbed, } \\
\dot{y}^{k+1}=\ddot{y}^{k}+h \lambda \dot{y}^{k} & \text { non-perturbed }
\end{array}
$$

Subtracting

$$
\hat{\epsilon}^{k+1}=\hat{\epsilon}^{k}+h \lambda \hat{\epsilon}^{k}=(1+h \lambda) \hat{\epsilon}^{k}
$$

Thus $\left|\hat{\epsilon}^{k}\right| \leq\left|\hat{\epsilon}^{0}\right|, \forall k \geq 0 \Rightarrow|1+h \lambda| \leq 1$, i e FE applied to $\{235\}$ with stepsize $h$ is stable $\forall \lambda \ni$

$$
|1+h \lambda| \leq 1
$$

The inequality (239) defines the stablaty iegron in the complex $h$ plane in which the method is stable

Sinilarly we can show that BE applied to $(235)$ with stepsize $h$ is stable $\forall \lambda \ni$

$$
|1-h \lambda| \geq 1
$$

\section{D.10 Stiffness}

When integrating a DE, one would expect the requisite stepsize to be relatively small in a region where the solution curve displays much variation, and to be relatively large where the solution curve straightens out to approach a line with slope nearly zero For some problems this is not the case Sometimes the stepsize is forced down to an unacceptably small level in a region where the solution curve is very smonth The phenomenon being exhibited here is known as stiffness In some cases we may have two different problems with the same solution, yet problem 1 is not stiff and problem 2 is stiff Clearly the phenomenon cannot he a function of the exact solution, since this is the same for both problems, and must be a property of the differential system itself $\mathrm{It}$ is thus more appropriate to talk of stiff syslems rather than of stiff problems

In this section we considel various aspects of the phenomenon of stiffness 'Phenomenon' is prohably a more appropriate word than 'property', since the latter rather implies that stiffness can be defined in precise mathematical terms, it turns ont not to be possible to do this in a satisfactory manner, evern for the restricted class of linear constant coefficient systens We shall also see several qualitative statements that $c$ an be (and mostly have been) 
made in an attempt to encapsulate the notion of stiflness, and state what is probably the most satisfactory of these as a 'definition' of stiffness

Before we address this issue, we will need to define some concepts C'onsider the linear constant coefficient inhomogeneous system

$$
\hat{y}^{\prime}=A \hat{y}+\phi(x)
$$

where $\hat{y}, \hat{\phi} \in \mathcal{R}^{n}$ and $A$ is a constant $n \times n$ matix with eigenvalues $\lambda_{t} \in \mathcal{C}, t=1,2, \quad, n$ (assumed distinct) and corresponding eigenvectors $\hat{c}_{t} \in \mathcal{C}^{n}, t=1,2, \quad, n$ The general solution of (241) takes the form

$$
\hat{y}(x)=\sum_{t=1}^{n} \kappa_{t} \exp \left(\lambda_{t} x\right) \hat{c}_{t}+\hat{\psi}(x)
$$

where the $\kappa_{t}$ are arbitrary constants and $\dot{\psi}(x)$ is a particular integral Now let us suppose that

$$
\operatorname{Re}\left(\lambda_{t}\right)<0, \quad t=1,2, \quad, n,
$$

which implies that each of the teims $\exp \left(\lambda_{t} x\right) \hat{c}_{t} \rightarrow 0$ as $x \rightarrow \infty$, so that the solution $\hat{y}(x)$ approaches $\dot{\psi}(x)$ asymptotically as $x \rightarrow \infty$, the term $\exp \left(\lambda_{t} x\right) \hat{c}_{t}$ will decay monotonic ally if $\lambda_{t}$ is real and sinusoidally if $\lambda_{t}$ is complex Interpreting $x$ to be time (as it often is in physical problems) it is appropriate to call $\sum_{t=1}^{n} \kappa_{t} \exp \left(\lambda_{t} x\right) \hat{c}_{t}$ the transtcnt solution and $\hat{\psi}(x)$ the steady-state solution If $\left|\operatorname{Re}\left(\lambda_{t}\right)\right|$ is large, then the conesponding term $\kappa_{t} \exp \left(\lambda_{t} x\right) \hat{c}_{t}$ will decay quickly as $x$ increases and is thus called a fast transient, if $\left|\operatorname{Re}\left(\lambda_{t}\right)\right|$ is small, the corresponding term $\kappa_{t} \exp \left(\lambda_{t} x\right) \hat{c}_{t}$ decays slowly and is called a slow transient L te.t $\bar{\lambda}, \underline{\lambda} \in\left\{\lambda_{t}, t=1,2, \quad, n\right\}$ be defined by

$$
|\operatorname{Re}(\bar{\lambda})| \geq\left|\operatorname{Re}\left(\lambda_{t}\right)\right| \geq|\operatorname{Re}(\underline{\lambda})|, \quad t=1,2, \quad, n
$$

so that $\kappa_{t} \exp (\bar{\lambda} x) \hat{c}_{t}$ is the fastest transient and $\kappa_{t} \exp (\underline{\lambda} x) \hat{c}_{t}$ the slowest We now define the stuffness ratio as

$$
\frac{|\operatorname{Re}(\bar{\lambda})|}{|\operatorname{Re}(\underline{\lambda})|}
$$

$J \mathrm{D}$ I ambert defines stiffness as follows

If a numerical method with a finite region of absolute stability, applied to a system with any ICs, is forced to use in a rertain interval of integr ation a steplength which is excessively small in relation to the smoothness of the exact solution in that interval, then the system is said to be stiff in that interval

There are other characteristics which are exhibited by many examples of stiff problems, but for each there are counterexamples, so these characteristics do not make good definitions of stiffness Nonetheless, definitions based upon these characteristics are in common use by some authors and are good clues as to the presence of stiffness Lambert refers to these as 'statements' rather than definitions, for the reasons stated above $A$ few of these are

Statement 1 A linear constant coeflicient system is stiff if all of its eigenvalues have negative real part and the stiffness ratio is large.

Statement 2 Stiffness occurs when stability requirements, rather than those of accuracy, constrain the steplength

Statement 3 Stiffness occurs when some components of the solution decay much more rapidly than others [14]

The origin of the term 'stiffress' seems to be somewhat of a mystery According to J O Hirschfelder, the term 'stiff' is used because such systems correspond to tight coupling

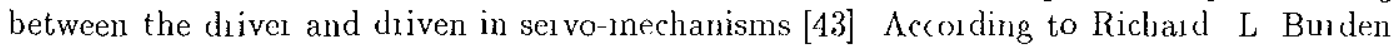
and J Douglas Faires, systems of DEs are called 'stift' (mathematical stiffness) due to their 
application in analyzing the motion of spring, damping and mass systems having large sping constants (physical stiffuess) [37] E g the IVP

$$
m \ddot{x}+c \dot{x}+k x=0, \quad x(0)=x_{0}, \quad \dot{x}(0)=0,
$$

with $m=1, c=1001, k=1000$, can be witten in the form (241) with $n=2$ and

$$
\begin{gathered}
A=\left(\begin{array}{rr}
0 & 1 \\
-1000 & -1001
\end{array}\right), \\
\phi(t)=\left(\begin{array}{l}
0 \\
0
\end{array}\right), \\
\ddot{x}(0)=\left(\begin{array}{c}
x_{0} \\
0
\end{array}\right),
\end{gathered}
$$

and has eigenvalues $\bar{\lambda}=-100(), \underline{\lambda}=-1$ Both eigenvalues have negative real pait and the stiffness ratio is

$$
\frac{|-1000|}{|-1|}=1000
$$

which is fairly large System (246) then certainly satisfies statements 1 \& 3 Here the spring constant $k$ is large and the damping constant $c$ is even larger [29] The exact solution to (246) is

$$
x(t)=x_{0}\left(-\frac{1}{999} e^{-1000 t}+\frac{1000}{999} e^{-t}\right) \approx x_{0} e^{-t}
$$

Note that (251) behaves quite nearly as a simple exponential $x_{0} \exp (-t)$, but the presence of the $\exp (-1000 t)$ term, even with a small coefficient is enough to make the numerical computation very sensitive to stepsize Stable integration of (246) requires a very small stepsize until well into the sinooth part of the solution curve, resulting in an error mum smaller than required lor acc uracy Thus the system also satisfies statement 2 and Lamberl's definition

\section{D.11 Functional Iteration}

A fixed pornt for a given function $\hat{g}$ is a value $\hat{p}$ for which

$$
\hat{g}(\hat{p})=\hat{p}
$$

Functional ateration or fised point teration consists of constructing a sequence

$$
\hat{p}^{n+1}=\hat{g}\left(\hat{p}^{n}\right)
$$

Problem (252) possesses a unique solution to which the iteration (253) will converge if $\hat{g}$ satisfies a Lipsehitz conclition in a neighborhood of $\hat{p} \quad[14]$

Certain problems, e g a root-finding problem $f(p)=0$ can be transformed into a fixed point problem such that repeated applications of (253) converge to the solution of the problem Root-finding problems and fixed-point problems are equivalent classes in the following sense. Given a root-finding problem $f(p)=0$ we can define a function $g$ with a fixed point at $p$ in a number of ways, $\mathrm{e} g$, as $g(x)=x-f(x)$ or as $g(x)=x+3 f(x)$ Conversely, if the function $g$ has a fixed point at $p$, then the function defined by $f(x)=x-g(x)$ has a zero at $p$ The fixed-point form of a problem is easier to analyze than the rout-finding form and certain fixed-point choices lead to very powerful soot-finding techniques [37]

E g to find a not of

$$
f(x)=\sin x
$$


in the interval $[1,6]$, we could iterate

$$
g_{1}(x)=x+\frac{1}{2} \sin x
$$

beginning at

$$
x^{0}=4000
$$

until a fixed point is approximated within a tolerance of $\epsilon=001$

$$
\begin{aligned}
& x^{0}=4000, \\
& x^{1}=3622, \\
& x^{2}=3391, \\
& x^{3}=3267, \\
& x^{4}=3205, \\
& x^{5}=3173, \\
& x^{6}=3157, \\
& x^{7}=3149
\end{aligned}
$$

$x^{7}$ then is within the specified toler ance of a root of (254)

Certain choices for $g$ converge faster than (255), e $g$

$$
y_{2}(x)=x+\sin x
$$

prodinces

$$
\begin{aligned}
& x^{0}=4000, \\
& x^{1}=3243, \\
& x^{2}=3142
\end{aligned}
$$

The linear systems arising from special cases of implicit numerical methods for ODEs and PDEs are generally solved by iterative methods

\section{D.12 Newton-Raphson Method}

Another iterative method for loot-finding is the Newton-Raphson method (NR), or simply Newton's method Suppose that $f$ is twice continuously differentiable on $[a, b]$ Let $\bar{x} \in[a, b]$ be an approximation to a root $p$ such that $f^{\prime}(\vec{x}) \neq 0$ and $\|\vec{x}-p\|$ is "small" Consider the fust Taylor polynomial for $f(x)$ expanded about $\bar{x}$,

$$
f(x)=f(\vec{x})+f^{\prime}(\ddot{x})(x-\bar{x})+\frac{f^{\prime \prime}(\xi(x))}{2}(x-\bar{x})^{2},
$$

where $\xi(x)$ lies between $x$ and $\bar{x}$ Since $f(p)=0$, this equation, with $x=p$, gives

$$
0=f(\bar{x})+f^{\prime}(\bar{x})(p-\bar{x})+\frac{f^{\prime \prime}(\xi(p))}{2}(p-\bar{x})^{2}
$$

Newton's method is derived by assuming that, since $\|p-\bar{x}\|$ is small, the term involving $(p-\bar{x})^{2}$ is negligible and that

$$
0 \approx f(\bar{x})+f^{\prime}(\bar{x})(p-\bar{x})
$$

Solving for $p$ in equation (262) gives

$$
p \approx \bar{x}-\frac{f(\bar{x})}{f^{\prime}(\bar{x})}
$$

This sets the stage for the Newton-Raphson method, which starts with an initial appioximation $p_{0}$ and generates the securence $p_{n}$, defined by

$$
p_{n} \approx p_{n-1}-\frac{f\left(p_{n-1}\right)}{f^{\prime}\left(p_{n-1}\right)}, \quad n \geq 1
$$

Newton's method is not guaranteed to converge, but when it does converge, it converges very quickly [37] 


\section{D.13 The Trapezoidal Rule}

Suppose that $f(x)$ is continuous for $a \leq x \leq b$, and let $a=x_{0}<x_{1}<x_{2}<<x_{n}=b$ be a subdivision of $[a, b]$ into $n$ equal intervals of length $h=(b-a) / n$ Then

$$
\int_{a}^{b} f(x) d x \approx \frac{h}{2}\left[f\left(x_{0}\right)+2 f\left(x_{1}\right)+2 f\left(x_{2}\right)+\quad+2 f\left(x_{n-1}\right)+f\left(x_{n}\right)\right]
$$

\section{D.14 Linear Multistep Methods}

Using the shortened notation

$$
\hat{f}^{n+j}=\hat{f}\left(\ddot{y}^{n+j}, t_{n+j}\right), \quad j=0,1, \quad, k
$$

we define a Lineat Multistep Method (LMM) or linear $k$-step method in standard form by

$$
\sum_{j=0}^{k} \alpha_{j} \dot{y}^{n+j}=h \sum_{j=0}^{k} \beta_{j} \hat{f}^{n+j},
$$

where $\alpha_{j}$ and $\beta_{j}$ are constants subject to the conditions

$$
\alpha_{k}=1, \quad\left|x_{0}\right|+\left|\beta_{0}\right| \neq 0
$$

The first of these conditions comoves, in a convenient manner, the arbitraniness that arises from the fact that we could multiply both sides of (267) by the same constant without altering the method The second condition prevents both $\alpha_{0}$ and $\beta_{0}$ being zelo, and thus precludes methods such as

$$
\dot{y}^{n+2}-\dot{y}^{n+1}=h \dot{f}^{n+1},
$$

which is essentially a 1-step and not a 2-step method, and is in practice indistinguishable from the 1-step method

$$
\ddot{y}^{n+1}-\ddot{y}^{n}=h \ddot{f}^{n}
$$

Method (270) is $\mathrm{FH}^{\mathrm{r}}$, the simplest of all nunierical methods for DEs [14] BE is then

$$
\hat{y}^{n+1}-\hat{y}^{n}=h \hat{j}^{n+1}
$$

Note that in (270), $\alpha_{k}=\alpha_{1}=1, \alpha_{0}=-1, \beta_{k}=\beta_{1}=0$, and $\beta_{0}=1$, and that in (271), $\alpha_{k}=a_{1}=1, \alpha_{0}=-1, \beta_{k}=\beta_{1}=1$, and $\beta_{0}=0$ In general, if $\beta_{k}=0$, the method is explicit, other wise the method is implic it

\section{D.15 Predictor-Corrector Methods}

Suppose that we wish to solve the standand IVP by an implicit LMM Then at earl step we have to solve for $\ddot{y}^{n+k}$ the implicit system

$$
\hat{y}^{n+k}+\sum_{j=0}^{k-1} \alpha_{j} y^{n+j}=h \beta \beta_{k} \hat{f}\left(\hat{y}^{n+k}, t_{n+k}\right)+h \sum_{j=0}^{n-1} \beta_{j} \hat{f}^{n+j}
$$

We normally do this by the fixed point iteration

$$
\begin{gathered}
y^{n+k, s+1}+\sum_{j=0}^{k-1} \alpha_{i} y^{n+j}=h \beta_{k} \hat{f}\left(y^{n+k, s}, t_{n+k}\right)+h \sum_{i=0}^{k-1} \beta_{j} \hat{f}^{n+j}, \\
y^{n+k, 0} \text { as bitialy, } \quad s=0,1,
\end{gathered}
$$


which will converge to the unique solution of (272) provided that

$$
h<\frac{1}{\left|\beta_{k}\right| \zeta},
$$

where $L$ is the Lipschitz constant of $f$ with respect to $y$ For non-stiff problems, this restriction on $h$ is not significant, in practice, considerations of arcuracy put a much more lestrictive constraint on $h$ Although (273) will converge for arbitıary $\dot{y}^{n+k_{1} 0}$, each iteration calls for one evaluation of the function $f$, and computation can obviously be saved if we can provide as good a guess as possible for $y^{n+k, 0}$ This is conveniently done by using a separate explicit LMM to provide the initial guess $\ddot{y}^{n+k, 0}$ We call this explicit method the preductor and the implicit method (272) the corrector, the two together comprise a Predictor-Corrector $\left(P C^{\prime}\right)$ pair $[14]$

\section{D.16 Adaptive Stepsize}

During the course of the integration of an IVP it is peiodically necessary to change the stepsize to minimize computational wolk while maintaining prescribed arcuracy [44] (and stability) Any worthwhile numerical ODE package varies the stepsize dynanically as it integrates, and its methods are called adaptrve stepsize methods In parts of the interval $\left(t_{0}, t_{N}\right)$ where the solution $\hat{y}(t)$ varics rapidly, we typically want smaller steps, whereas in parts where the solution in nearly constant, we usually want larger steps In practice, we have a parameter $\epsilon$ and we want $\left\|d^{n}\right\| \leq \epsilon$, for each step we lake Of course we would like to take as hig a step as we can One arlaptive stepsize method estimates the LTE $\hat{d}^{n}(h)$ as

$$
\hat{d}^{n}(h)=\frac{\hat{y}^{n+2,2 h}-\hat{y}^{n+2, h}}{2^{2}-1}
$$

where $r$ is the order of the numerical method, $h$ is the current stepsize, and $y^{i+2,2 h}$ and $y^{n+2, h}$ are approximations of $y\left(t_{n+2}\right)$ using stepsizes of $2 h$ and $h$, resperlively, and estimates the desired stepsize $h^{*}$ as

$$
h^{*}=h\left(\frac{\epsilon}{\| \hat{d^{n}(h) \|}}\right)^{1 /(1+1)},
$$

where $\epsilon$ is the tolerance, and then does the following

1 if $h \leq h^{*}$, then set $\hat{y}^{n+2}=\ddot{y}^{n+2, h}$ and proceed with $h^{*}$ as the cunent stepsize,

2 other wise, set $h=h^{*}$ and letry the combination

\section{17 Runge-Kutta Methods}

The simplest of all numerical methods is $\mathrm{FE}$,

$$
\hat{y}^{n+1}=\hat{y}^{n}+h \hat{f}^{n}
$$

FE is linear in $\hat{y}^{\text {n }}$ and $\hat{f}^{n}$ and, being a one-step method, presents no difficulty when we want to change the steplength, but of course it has very low accutacy Linear multistep methods achieve higher accuracy by retaining linearity with respect to $\hat{y}^{n+j}$ and $f^{n+j}, j=0,1, \quad, k$, but sacrificing the one-step format The result of retaining the linearity is that the local crrot has a relatively simple structure, which is why we are able to estimate it so easily, the cost of moving to a multistep format is the considerable difficulties encountered when we want to change steplength

Runge-Isutta (RK) methods develop from Euler's Rule in exactly the opposite direction, higher order is achieved by retaining the one-step form but sarrificing the linearity The 
result is that there is no difficulty in changing the steplength, but the structure of the local error is much more complicated, and there exists no easy and cheap error estimate We are sather in a Catch-22 situation, with linear multistep methods it is easy to tell when we ought to change steplength but hard to change it, while with Runge-Kutta Methods it is haul to tell when to change steplength but easy to change it!

The general s-stage Runge-Kutta method for the IVP

$$
\grave{y}^{\prime}=\hat{f}(x, y), \quad y(a)=\hat{\eta}, \quad \hat{f} \mathcal{R} \times \mathcal{R}^{m} \rightarrow \mathcal{R}^{m}
$$

is defined by

$$
\left.\begin{array}{l}
\hat{y}^{n+1}=\ddot{y}^{n}+h \sum_{i=1}^{s} b_{i} k_{i}, \\
\hat{k}_{i}=\hat{f}\left(\hat{x}^{n}+c_{i} h, \dot{y}^{2 z}+h \sum_{j=1}^{s} a_{i j} \hat{k}_{j}\right), \quad i=1,2, \quad, s
\end{array}\right\}
$$

We shall always assume that the following (the row-sum condition) holds [14]

$$
c_{i}=\sum_{j=1}^{s} a_{i j}, \quad i=1,2, \quad, s
$$

$\mathrm{E} \mathrm{g}$ the classical 4th-order RK method is

$$
\left\{\begin{array}{l}
\hat{k}_{1}=h \hat{f}\left(\ddot{y}^{n}, t_{n}\right), \\
\dot{k}_{2}=h \hat{f}\left(\dot{y}^{n}+\frac{1}{2} \dot{k}_{1}, t_{n}+\frac{1}{2} h\right), \\
\hat{k}_{3}=h \hat{f}\left(\hat{y}^{n}+\frac{1}{2} \hat{k}_{2}, t_{n}+\frac{1}{2} h\right), \\
\hat{k}_{4}=h \hat{f}\left(\hat{y}^{n}+\hat{k}_{3}, t_{n}+h\right), \\
\ddot{y}^{n+1}=\ddot{y}^{n}+\frac{1}{6}\left(\dot{k}_{1}+2 \hat{k}_{2}+2 \hat{k}_{3}+\dot{k}_{4}\right)
\end{array}\right.
$$

\section{D.18 Iterative Techniques for Linear Systems}

Numerical methods which yield solutions after an amount of computation that can be speciffed in advance are called drrect methods An example of a direct method for approximating the solution of a system of linear equations is Gauss clmmination We will not pussue dinect methods here In contrast, an indirect method or iterative method is one in which we start flom an approximation to the true solution and, if successful, ohtain bettel and better approximations fiom a computational cy cle repeated as often as may be necessary for arhieving a requiled accuracy, so that the amount of arithmetic depends upon the accuracy required

Iterative methods are used mainly in those problems for which convergence is known to be 1 apid, so that the solution is obtained with much less wolk than that of a direct method, and for systems of large order but with many zero coefficients, the so-called "sparse" systems, tor which elinination methods would be relatively laborious and need much storage Such systems occur in connection with vibrational problems, BVPs, PDEs, circuit analysis, and other applications and have given rise to a rapidly giowing literature [29]

An iterative technique to solve the linear system $A \hat{x}=\hat{b}$ begins with an initial approximation $\hat{x}^{0}$ to the solution $\hat{x}$, and gener ates a sequence of vectors $\left\{\hat{x}^{k}\right\}_{k=0}^{\infty}$ which converges to $\hat{x}$ Iterative techniques involve a process that conver ts the system $A \hat{x}=\hat{b}$ into an equivalent system of the form $\hat{x}=T \ddot{x}+\hat{c}$ for some matrix $T$ and vector $\hat{c}$ Aftel the initial vector $\hat{x}^{0}$ is selected, the sequence of approximate solution vectors is generated by computing

$$
\hat{x}^{k}=T \hat{x}^{k-1}+\hat{c}, \quad k=1,2,
$$

This is a form of fixed-point iteration [37] 


\section{18.1 Jacobi Iteration}

The linear system $A \hat{x}=\hat{b}$ given by

$$
\begin{array}{lr}
E_{1} & 10 x_{1}+5 x_{2}+0 x_{3}=-5 \\
E_{2} & 2 x_{1}+30 x_{2}+-4 x_{3}=24 \\
E_{3} & 0 x_{1}+3 x_{2}+20 x_{3}=23
\end{array}
$$

can be converted to the form $\ddot{x}=T \ddot{x}+\ddot{c}$ as follows Solve each equation $E_{i}$ for $x_{i}$ to obtain

$$
\begin{aligned}
& x_{1}=-\begin{array}{ll}
\frac{1}{2} x_{2} & -\frac{1}{2}
\end{array} \\
& x_{2}=-\frac{1}{15} x_{1}+\frac{2}{15} x_{3}+\frac{4}{5} \\
& x_{3}=\quad-\frac{3}{20} x_{2}+\frac{23}{20}
\end{aligned}
$$

In this cxample,

$$
T=\left(\begin{array}{rrr}
0 & -\frac{1}{2} & 0 \\
-\frac{1}{15} & 0 & \frac{2}{15} \\
0 & -\frac{3}{20} & 0
\end{array}\right) \quad \text { and } \quad \hat{c}=\left(\begin{array}{r}
-\frac{1}{2} \\
\frac{4}{5} \\
\frac{23}{20}
\end{array}\right)
$$

Fol an initial approximation, we let $\hat{x}^{0}=(0000,0000,0000)^{T}$ and generate $\hat{x}^{1}$ by

$$
\begin{aligned}
& x_{1}^{1}=-\frac{1}{2} x_{2}^{0}-\quad-\frac{1}{2}=-0500, \\
& x_{2}^{1}=-\frac{1}{15} x_{1}^{0}+\frac{2}{15} x_{3}^{0}+\frac{4}{5}=0800, \\
& x_{3}^{1}=\quad-\frac{3}{20} x_{2}^{0}+\frac{23}{20}=1150
\end{aligned}
$$

Additional iterates $\hat{x}^{k}=\left(x_{1}^{k}, x_{2}^{k}, x_{3}^{k}\right)^{T}$ are generated in a similan manner and to 3 decimal places are

$$
\begin{aligned}
& \hat{x}^{0}=\left(\begin{array}{lll}
0000, & 0000, & 0000
\end{array}\right)^{T}, \\
& \hat{x}^{1}=\left(\begin{array}{lll}
-0500, & 0800, & 1150
\end{array}\right)^{T}, \\
& \hat{x}^{2}=\left(\begin{array}{lll}
-0900, & 0987, & 1030
\end{array}\right)^{T}, \\
& \hat{x}^{3}=\left(\begin{array}{lll}
-0993, & 0997, & 1002
\end{array}\right)^{T}, \\
& \ddot{x}^{4}=\left(\begin{array}{lll}
-0999, & 1000, & 1000
\end{array}\right)^{I}, \\
& x^{5}=\left(\begin{array}{lll}
-1000, & 1000, & 1000
\end{array}\right)^{T}
\end{aligned}
$$

Note that this method converges to the solution

$$
\hat{x}=(-1000,1000,1000)^{T}
$$

of (283) to an accunacy of 3 decimal places in 5 iterations We usıally itex ate until a fixed nunber of iterations ate complete, or until

$$
\frac{\left\|\hat{x}^{n+1}-\hat{x}^{n}\right\|}{\left\|\hat{x}^{n+1}\right\|}<\epsilon,
$$

for some sperified roles ance $\epsilon>0$

This method is called the Jacobr aterative method [37] Note that Jacobi iteration is a onestep methud The system (283) was contrived to converge quickly in order to conveniently illustrate the method In prartice, Jacohi iteration applied to a particular problem may conves ge very slowly, or may diverge altogether E g Jacobi iteration applied to the system

$$
\begin{aligned}
& F_{1} 9 x_{1}+7 x_{2}+0 x_{3}=66 \\
& E_{2} 3 x_{1}+4 x_{2}+1 x_{3}=28 \\
& E_{3} 0 x_{1}+3 x_{2}+5 x_{3}=14
\end{aligned}
$$


converges, to 3 decimal places, to the solution

$$
\hat{x}=(5000,3000,1000)^{T}
$$

in 59 iterations Jacobi iteration applied to the system

$$
\begin{array}{rrr}
E_{1} & -14 x_{1}+-91 x_{2}+52 x_{3}=4617 \\
E_{2}-68 x_{1}+79 x_{2}+76 x_{3}=-649 \\
E_{3} \quad 64 x_{1}+16 x_{2}+83 x_{3}=-4872
\end{array}
$$

does not converge at all Also, in practice, the linear system is often much langer than the $3 \times 3$ systems illustrated heie

The Jacobi iterative method is called a method of smmultaneous corrections because no element $x_{i}^{k}$ of an approximation $\ddot{x}^{k}$ is used until all the elements of $\hat{x}^{k}$ have been calculated [29] Jacoli iteration is not very fast in itself, but simple and rompletely parallel, and thus very fast when implemented on a parallel computer architecture

\section{D.18 2 Gauss-Seidel Iteration}

An inmprovement oves Jacobi iteralion is suggested by an analysis of (284) To compute $x_{i}^{k}$, the components of $x^{k-1}$ ale used Since, $f \circ i>1, x_{1}^{k}, \quad, x_{i-1}^{k}$ have already been computed and are likely to be hetter approximations to the actual solutions $x_{1}, \quad, x_{i-1}$ than $x_{1}^{k-1}, \quad, x_{i-1}^{k-1}$, it seems reasunable to compute $x_{i}^{k}$ using these most recently calculated values This is called the Crauss-S'Seidel iterative technique (GS) [37] The system (283) is then jterated as

$$
\begin{aligned}
& x_{1}^{k}=-\frac{1}{2} x_{2}^{k-1} \quad-\frac{1}{2} \\
& x_{2}^{k}=-\frac{1}{15} x_{1}^{k}+\frac{2}{15} x_{3}^{k-1}+\frac{4}{5} \\
& x_{3}^{k}=\quad-\frac{3}{20} x_{2}^{k}+\frac{23}{20}
\end{aligned}
$$

Letting $\ddot{x}^{0}=(0000,0000,0000)^{T}$, the iterates generated to 3 decinal places are

$$
\begin{aligned}
& \hat{x}^{0}=\left(\begin{array}{lll}
0000, & 0000, & 0000
\end{array}\right)^{T}, \\
& \hat{x}^{1}=\left(\begin{array}{lll}
-0500, & 0833, & 1025
\end{array}\right)^{T}, \\
& \hat{x}^{2}=\left(\begin{array}{lll}
-0917, & 0998, & 1000
\end{array}\right)^{T}, \\
& \hat{x}^{3}=\left(\begin{array}{lll}
-0999, & 1000, & 1000
\end{array}\right)^{T}, \\
& \ddot{x}^{4}=\left(\begin{array}{lll}
-1000, & 1000, & 1000)^{T}
\end{array}\right.
\end{aligned}
$$

Note that this nuethod converges to the solution

$$
\hat{x}=(-1000,1000,1000)^{I}
$$

of (283) to an accuracy of 3 decimal places in 4 itesations a $20 \%$ saving over Jacobi iteration Gauss-Seidel iteration applied to (290) converges, to 3 decimal places, to the solution

$$
\ddot{x}=(5000,3000,1000)^{T}
$$

in 28 iterations a $53 \%$ saving oves Jacolvi iteration

Gauss-Seidel is a method of successme rorrections because each element $x_{i}^{k}$ of an approximation $\hat{x}^{k}$ is used as soon as it is calculated [29] Gauss-Seidel itelation is not obviously parallel, but when used to solve PDEs, theıe is much parallelisn hidden in it 


\section{D.18.3 Relaxation Method}

In Jacobi iteration, rather than correct $\hat{x}^{k}$ using the Jacobi correction $\hat{x}^{k+1, J}$, defined by

$$
\hat{x}^{k+1, J}=T \hat{x}^{k}+\hat{c},
$$

which can over-correct, lesulting in oscillatory behavior which may letard convergence, we can use a weighted average of this computed value and the previous iterate,

$$
\hat{x}^{k+1}=\omega \hat{x}^{k+1, J}+(1-\omega) \hat{x}^{k},
$$

in hopes of damping out the oscillations and accelerating convergence 'This technique can be applied similarly to (rauss-Seidel iteration Methods employing this wcighted avcrage are called relaxation methods For choices of the weighting factor $0<\omega<1$, the procedures are called under-relaxation methods For choires $1<\omega$, the procedures are ralled overrelaxation methods These methods are abbreviated SOR for Successive Over-Reluxation and are particularly useful for solving the linear systems that occur in the numerical sulution of certain PDEs [37]

Ovel-relaxation can increase the eflects of iteration and make a stable method converge. faster E $\mathrm{g}$ relaxed Gauss-Seidel with $\omega=134$ applied to $(290)$ converges, to 3 decimal places, to the solution

$$
\hat{x}=(5000,3000,1000)^{T}
$$

in 9 iterations a $68 \%$ saving over Gauss-Seidel iteration, an $85 \%$ saving over Jacobi iter ation

Under-relaxation can decrease the efferts of iteration and make an otherwise unstable method stable Eg relaxed Ganss-Scidel with $\omega=05$ applied to (292) converges, to 3 decimal places, to the solution

$$
\ddot{x}=(-54000,-47000,-8000)^{T}
$$

in 38 iterations a problem for which Jarobi and Crauss-Seidel do not converge at all

\section{184 Matıix-Splitting}

A decomposition of a matrix $A$ into a sum of matrices, which simplifies manipulation of $A \ddot{x}=\hat{b}$ is called a matrix spluttung $\mathrm{E} g$ let

$$
\begin{aligned}
-U & =\text { strictly upper triangular part of } A, \\
D & =\text { diagonal part of } A, \\
-L & =\text { striclly lower triangular part of } A,
\end{aligned}
$$

then the matrix $A$ is split into simpler matrices as

$$
A=D-U-L
$$

The Jacobi method can then be represented in matrix form as a splitting

$$
\hat{x}^{k+1}=D^{-1}(I+L) \hat{x}^{k}+D^{-1} \hat{b}
$$

the Gauss-Seidel splitting is

$$
\hat{x}^{k+1}=(D-L)^{-1}\left(U \hat{x}^{k}+(D-L)^{-1} \dot{b},\right.
$$

and the relaxed ('auss-Sejdel splitting is [37]

$$
x^{k+1}=(D-\omega L)^{-1}[(1-\omega) D+\omega U] \hat{x}^{k}+\omega(D-\omega L)^{-1} b
$$




\section{D.19 Subroutine Packages}

Colleclions of subıoutines for solving systems of ODEs, called subroutine packages, or solvers, etc, are available The Livermore Solver for Ordinary Differential Equations (LSODE) is designed to produce numerical solutions to IVPs, especially for stiff chernical kinetics problenıs This FORTRAN-callable packagc, witten by Alan C Hindmaisch at Lawrence Livermore National Laboratory (LLNL), uses the backward-difference formula (BDF) method, and implicit Adams methods [44]

Another FORTRAN collection is CHEMSODE a stuff ODE solver for the equatzons of chemical kinetics, developed by Colin J Aro at LLNI, This package implements three different types of preconditioned time diflercncing techniques, along with a choice of steplength control, which eliminate the need for the matrix algebra typically assuciated with implicit numerical methods, while setaining the numerical stability [10] 


\section{E Parallel Processing}

Some terminology from Parallel Processing is defined here

$1 C P U$ - Central Processing Unit, a single computer

$2 O S$ - Operating System

3 bandwidth - the bandwidth of a memory system is the maximum number of bytes that can he transferted in one second between the processing conıponent and the memory 'This concept can be extended to describe the rate at which other system components involved in the transport of data can operate [45]

4 pipelining - the process of putting portions of a problem into an assembly line $\mathrm{F}, \mathrm{g}$ consider adding two floating point numbers $a=10123 \mathrm{e}-3, b=10000 \mathrm{e}-1$ We can perform this addition in stages

(a) Shift so adix points align

$$
\begin{aligned}
& 0010123 \mathrm{e}-1 \\
& 1000000 \mathrm{e}-1
\end{aligned}
$$

(b) Aild

$$
\begin{array}{r}
0010123 \mathrm{e}-1 \\
+1000000 \mathrm{e}-1 \\
\hline 1010123 \mathrm{e}-1
\end{array}
$$

(c) Shift

$$
01010123
$$

(d) Round

$$
010101
$$

We can now use the 4-stage scheme above to add two floating point vectors $\hat{a}=$ $\left({ }_{1} a, \quad, N_{N} a\right)^{T}$ and $\hat{b}=\left({ }_{1} b, \quad,{ }_{N} b\right)^{T}$ component-wise I e while ${ }_{i} a$ and ${ }_{i} b$ are being shifted, $i_{-1} a$ and $i_{i-1} b$ are being added, ${ }_{i-2} a+{ }_{i-2} b$ is being shifted, ${ }_{i-3} a+{ }_{i-3} b$ is being lounded, ${ }_{i-4} a+{ }_{i-4} b$ is being output The total time $t$ required to add two vectors of length $N$ is

$$
t=t_{s}+t_{l}(N-1)
$$

where $t_{s}$ is the setup time iequired to get the first result (i e for the first compronent sum, ${ }_{1} a+{ }_{1} b$ to pass completely through the pipeline), and $t_{l}$ is the time required tor the longest (slowest) operation in the pipeline Pipelining is also ralled vector processing

5 vector machıne - a computer implementing the pipelining paradigm

6 parallelism - the process of performing tasks concurrently, the degrce to which no1tions of a problem can be processed concuirently

7 problem size - the number of pieces of data that we want to update.

8 parallel machine - a computer consisting of two or more (CPUs connected via some interconnection network, a collection of inultiple processors that work on a single problem Such a computer system is capable of achieving tremendous cost/peiformance benefits Parallel processols are classified as either shared memory or distributed memory machines Machines consisting of 1,000,000 or more CPUs are called massively parallel computers 
9 parallel wall clock time, $t_{p}$ - the maximum time for a CPU in a parallel machine to complete its portion of the problem If $t_{i}$ is the time required for CPU $i$ to complete, and there are $m$ CPUs, then $t_{p}=\max _{i=1}^{m} t_{i} \quad t_{p}$ is essentially the time requined for a parallel machine to complete the problein

10 serial wall clock tıme, $t_{s}$ - the total time for all C'PUs in a parallel machine to complete their portions of the problem $t_{s}=\sum_{i=1}^{m} t_{i} \quad t_{s}$ is essentially the time that would be. required for a single machine to complete all portions of the problem sequentially

11 A node in a network consists of a processor and its local memory The size $n$ of a netwolk is the number of nodes it contains A partition is a particular scheme for dividing a plohlem into portions, or a cor responding configuration of nodes in a parallel processol

12 speedup $-t_{s} / t_{p}$

$13 R P$ - Relative Pelfornance $R P=\left(t_{s} / t_{p}\right) / n \quad R P$ is essentially the efficiency of the parallelization of the problem If the problem is divided evenly hetween CPUs, then $R P=1$ If $R P=1$, then we have a peifectly scalable problem A value of $R P>1$ is said to be superlinear If dividing the problem into snall portions results in the problem fitting completely into the CPI Is' local rache memolies, then we have eliminated the time spent in communication with slow memory, and not replaced it with time spent in message-passing Thus we have $t_{p}\left\langle t_{s} / n\right.$, and therefore $R P>1$ For performance vs processors, any value of $R P$ betser than 1 is a good retum

14 load balancong - sharing the processing burlen equally across all C'PUs in a parallel machine, to minimize $t_{p}$ The goal of load balancing is to keep processor nodes busy and have them finish roughly at the same time Valuable processor cycles are wasted if some nodes have to wait on others to finish The greatest speedup is possible only when all processors are busy all of the time

15 SIMD - Single-Instruction Multi-Data All CPUs execute the same instruction simultaneously In the SIMD paradigm, all (:PUs do the same thing at the same time or else they remain idle $\mathrm{Eg}$ a vector processor

16 MIMD - Multi-Instruction Multi-Data Many CPUs can be simultaneonsly executing different instructions on different data Futhermor, these CPUs operate in a largely autonomous manner as though they were separate computers

17 interconnection network (IN) topology - a mapping function fiom the set of processors and memories onlo the same set of processors and memories

18 A shared memory architecture is one which arcomplishes interprocessor coordination through a glohal memory shared by all C'PUs (cf distributed memory)

19 rache memory - a special-pupose rantom access nemoly designed to reduce a number of contention problems which arise from a bus-structured shared-memon IN topology

20 In a shared memory arrhitecture, each C'PU has its own local cache A typical busstructured parallel computer attempts to reduce contention for the bus by fetching instructions and data directly fiom each individual (ache, as much as possible

21 MPI - Message-Passing Interface A distuibuted-nnemory IN consists of processors and thein local memonies connected by communication links There is no global memory so it is necessary to inove data from one local memory to another by means of message-passing This is typically dune by a SENI)/RECEIVE pain of commands 
which must be written into the application software by a programmer Thus, programmers must learn the message-passing paradigm, and cope with the details of data copying and the attendant consistency issues

22 A distributed memory architecture ty pically combines a local memory and a processor at each node of the IN Message-passing is used to communicate between any two CPUs, and there is no global, shared memory (cf shared memory)

23 Melko C\$-2 - a distributed memory system Communication must be requested no shared memory $[46,27]$ 


\section{F Related Methods}

\section{F.1 Introduction}

Several mumerical methods are in use which although not germane to our discussion, are often used altemately on sinilar problems These related methods are presented here for comparison

\section{F.2 Finite Difference Approximations to Derivatives.}

When a function $F$ and its derivatives are single-valued, finite and continuous functions of $x$, then by 'Taylor's theorem,

$$
F(x+h)=F(x)+h F^{\prime}(x)+\frac{1}{2} h^{2} F^{\prime \prime}(x)+\frac{1}{6} h^{3} F^{\prime \prime \prime}(x)+
$$

and

$$
F(x-h)=F(x)-h F^{\prime}(x)+\frac{1}{2} h^{2} F^{\prime \prime}(x)-\frac{1}{6} h^{3} F^{\prime \prime \prime}(x)+
$$

Addition of these expansions gives

$$
F(x+h)+F(x-h)=2 F(x)+h^{2} F^{\prime \prime}(x)+O\left(h^{1}\right),
$$

where $O\left(h^{4}\right)$ denotes terms containing fourth and higher powers of $h$ Assuming these are negligible in comparison with lower powers of $h$ it follows that,

$$
F^{\prime \prime}(x)=\left(\frac{d^{2} F^{2}}{d x^{2}}\right)_{x=x} \approx \frac{1}{h^{2}}\{F(x+h)-2 F(x)+F(x-h)\},
$$

with a leading enol on the right-hand side of onder $h^{2}$

Subtraction of equation (312) from equation (311) and neglest of terms of order $h^{3}$ leads to

$$
F^{\prime}(x)=\left(\frac{d F}{d x}\right)_{x=x} \approx \frac{1}{2 h}\{F(x+h)-F(x-h)\},
$$

with an entor of onder $h^{2}$

Let $A=(x-h, u(x-h)), P=(x, u(x)), B=(x+h, u(x+h))$ Then (315) clearly approximates the slope of the tangent at $P$ by the slope of the chord $A B$, and is called a centsal-difference approximation We can also approximate the slope of the tangent at $P$ by either the slope of the chord $P B$, giving the for ward-difference formula,

$$
F^{\prime}(x) \approx \frac{1}{h}\{F(x+h)-F(x)\},
$$

or the slope of the chord $A P$ giving the backward-difference formula (BDF)

$$
F^{\prime}(x) \approx \frac{1}{h}\{F(x)-F(x-h)\},
$$

Both (316) and (317) can be derived immediately from (311) and (312) respectively, assuming second and higher powers of $h$ are negligible This shows that the learling errors in these for ward and backward-difterence formulae are both $O(h)$ [40]

\section{F.3 Finite Difference Method}

In the Fonte Difference Method ( $F D M$ ), derivatives at a point are appioximated by difference quoticnts over a sinall interval $\mathrm{E} g$ for the 1-D diffusion equation,

$$
u_{t}=D u_{x x},
$$


we set up a grid in the $x$ - $t$ plane with grid spacings $\Delta x$ and $\Delta t$ The basis of the simplest finite diflerence method is to replace the second derivative on the right-hand side of (318) by a central difference quotient in $x$ : and replace $u_{t}$ by a for ward difference in time Then, one advances the approximate solution forward in time, one time level after another More precisely, if we let $u_{j}^{n}$ denote the approximate solution at $x_{j}=j \Delta x$ and $t_{n}=n \Delta t$, then the finite difference analog of (318) is

$$
\frac{u_{j}^{n+1}-u_{j}^{n}}{\Delta t}=\frac{D}{(\Delta x)^{2}}\left(u_{j+1}^{n}-2 u_{j}^{n}+u_{j-1}^{n}\right)
$$

Ol

$$
u_{j}^{n+1}=u_{j}^{n}+\mu\left(u_{j+1}^{n}-2 u_{j}^{n}+u_{j-1}^{n}\right), \quad j=1, \quad, q
$$

where

$$
\mu=\frac{D \Delta t}{(\Delta: t)^{2}}
$$

Generalization to more than one spatial dimension is a natural extension of (320)

The classical stability conditon for FDM is

$$
\Delta t \leq \frac{(\Delta x)^{2}}{2 D}
$$

and is slightly stronger than the actual stability iestriction which can be delived for $(320)$ $[20]$

Note that unce the right-hand side of (318) is approximated by a central difference quotient, $u_{t}$ can be approximated by, e g a forward difference (explicit method), a backward difference (implicit method), the Numerical Method of Lines, a Preconditioned Time Difference, etc wherein different numerical methords arise

\section{F.4 Finite Element Method}

The Finile Element Melhod (FEM) was originally developed for use in civil engineering but is now used for approximating the solutions to partial differential equations that ar ise in all areas of applied mathematics One advantage of the finite elcment method oves finite clifference methods is the relative ease with which the houndary conditions of the problem are handled Many physical problems have houndary conditions involving derivatives and in egularly shaped boundaries Boundary conditions of this type are difficult to handle using finite difference techniques since each boundary condition involving a derivative must be approximated by a difference quotient at the gridpoints, and in egular shaping of the boundary makes placing the giidpoints difficult The finite element method includes the boundary conditions as integrals in a functional that, is being minimized, so the construction procedure is indepeudent of the particular boundary conditions of the problem

Consider, eg the partial differential equation

$$
\frac{\partial}{\partial x}\left(p(x, y) \frac{\partial u}{\partial x}\right)+\frac{\partial}{\partial y}\left(q(x, y) \frac{\partial u}{\partial y}\right)+\jmath(x, y) u(x, y)=f(x, y)
$$

with $(x, y) \in \mathcal{D}$, where $\mathcal{D}$ is a plane region with boundary $\mathcal{S}$

Boundary conditions of the form

$$
u(x, y)=g(x, y)
$$

are imposed on a portion $\mathcal{S}_{1}$ of the boundary On the iemainder of the boundary $\mathcal{S}_{2}, u(x, y)$ is required to satisfy

$$
p(x, y) \frac{\partial u}{\partial x}(x, y) \cos \theta_{1}+q(x, y) \frac{\partial u}{\partial y}(x, y) \cos \theta_{2}+y_{1}(x, y) u(x, y)=y_{2}(x, y)
$$


where $\theta_{1}$ and $\theta_{2}$ are the direction angles of the outward normal to the boundary at the point $(x, y)$

Physical problems in the areas of solid mechanics and elasticity have associated partial differential equations similar to (323) The solution to a problem of this type is typically the mininization of a cestain functional, involving integrals, over a class of functions determined by the pioblem

Suppose $p, q, r$, and $f$ are all continuous in $\mathcal{D} \cup \mathcal{S}, p$ and $q$ have continuous first partial derivatives, and $g_{1}$ and $g_{2}$ are continuous on $\mathcal{S}_{2}$ Suppose, in addition, that $p(x, y)>0$, $q(x, y)>0, r(x, y) \leq 0$, and $g_{1}(x, y)>0$ Then a solution to (323) uniquely minimizes the functional

$$
\begin{aligned}
I[w]= & \iint_{\Gamma}\left\{\frac{1}{2}\left[p(x, y)\left(\frac{\partial w}{\partial x}\right)^{2}+q(x, y)\left(\frac{\partial w}{\partial y}\right)^{2}-r(x, y) w^{2}\right]+f(x, y) w\right\} d x d y \\
& +\int_{\mathcal{S}_{2}}\left\{-g_{2}(x, y) w+\frac{1}{2} g_{1}(x, y) w^{2}\right\} d S
\end{aligned}
$$

over all functions $w$ satisfying (324) on $\mathcal{S}_{1}$ which are twice continuously differentiable The finite element method approximates this solution by minimizing the functional $I$ over a smalle1 class of functions

The first step is to divide the recion into a finite number of sections, or elements, of a rcgular shape, either rectangles or triangles

The set of functions used for approximation is generally a set of piecewise polynomials of fixed degree in $x$ and $y$, and the approximation requires that the polynomials be pieced together in such a mannes that the resulting function is continuous with an integrable or continuous first or second derivative on the entire region Polynonials of linear type in $x$ and $y$

$$
\phi(x, y)=a+b x+c y,
$$

are commonly used with triangular elements, while polynomials of bilineas type in $x$ and $y$,

$$
\phi(x, y)=a+b x+c y+d x y,
$$

ase used with rectangular elements [37]

\section{F.5 Numerical Method of Lines}

To solve the 1-D pure diffusion equation

$$
u_{t}=D u_{x x},
$$

with Initıal Condetions (IC)

$$
u(x, 0)=\sin (\pi x / L),
$$

and Boundary ('onditions (B(')

$$
u(0, t)=u(L, t)=0
$$

we could replace the partial derivatives with alyebraic approximatıons evaluated at a general point with indices $(j, n)$, this would lead to a set of algebraic equations that approximate equation (329) Once the approximation algebraic equations have been defined, they could be solved using any standard linear equation solver to obtain an approxumate numerical solution to equation (329), of couse, the anxiliany conditions, equations (330-331), would also have to be inr luded in the algebrair equations This proredure is the basis for well-known classical finite difference and finite element methods for PDEs 'The Numerical Method of Lines (NHMOI) is really just a suall departure from this basic approach In the NUMOL, 
we retain the index $j$ to account for variations of $u$ with $x$, but we treat $t$ as a continuous variable (rather than $t$ evaluated at discrete points consesponding to the index $n$ ) Thus, we will replace the partial derivative $u_{x x}$ in equation (329) with an algebraic approximation evaluated at point $j$, but keep the derivative $u_{t}$, this will lead to a system of differential equations in $t$, and since we now have only one independent variable $t$, the differential equations will be ODEs This is the essence of the NUMOL [16]

\section{F.6 Method of Characteristics}

Consider the equation

$$
a \frac{\partial u}{\partial x}+b \frac{\partial u}{\partial y}=c
$$

where $a, b$, and $c$ are, in general, functions of $x, y$, and $u$ but not of $\partial u / \partial x$ and $\partial u / \partial y$, i e the first-order derivatives occur only to the first degree although the equation need not be linear in $u$ Such an equation is said to he quasi-linear It is customaty to put $\partial u / \partial x=p$ and $\partial u / \partial y=q$ and to wite the equation as

$$
a p+b q=c
$$

At each point of the solution domain of such an equation there is a dinertion along which the integration of equation (333) transforms to the integration of an ordinary differcntial equation In other words, in this directzon the expr ession to be integrated will be independent of partial derivatives in other directions, such as $p$ and $q$

Assume we know the solution values $u$ of equation (333) at every point on a rurve $C$ in the $x-y$ plane, where $C$ ' does not coincide with the curve $\Gamma$ on which initial values of $u$ are specified The question to he askerl at this stage is this "Can we determine values for $p$ and $q$ on $C$ from the values of $u$ on $C$ so that they salisfy equation (333)?"

If we can, then in directions tangential to $C^{\prime}$ from points on ${ }^{\prime}$ we shall automatically sat isfy the differential relationship

$$
d u=\frac{\partial u}{\partial x} d x+\frac{\partial u}{\partial y} d y=p d x+q d y
$$

where $d y / d x$ is the slope of the tangent to $\left(;\right.$ at $P(x, y)$ on $C^{-}$

Equations (333-334) are two equations for $p$ and $q$ Elimination of $p$ between 1 hen gives

$$
d u=\frac{c-b q}{a} d x+q d y
$$

which can be written as

$$
q(a d y-b d x)+(c d x-u d u)=0
$$

This equation is explicitly independent of $p$ because the confficients $a, b$ and $c$ are functions of $x, y$ and $u$ only It can also be made independent of $q$ by choosing the curve $C$ so that its slope $d y / d x$ satisfies the equation

$$
a d y-b d x=0
$$

By equations (336-337) it then follows that

$$
c d x-a d u=0
$$

Equation (337) is a differential equation for the curve $C$ and (338) is a differential equation for the solution values of $u$ along $C$ The curve $C$ is called a characteristac curne or charactersstıc These equations are easy to remember because they can be witten as

$$
\frac{d x}{a}=\frac{d y}{b}=\frac{d u}{c}
$$

This also shows that $u$ may be found fiom either the equation $d u=(c / a) d x$ or the equation $d u=(c / b) d y \quad[40]$ 


\section{F.7 Particle Methods}

Snoothed Particle Hydrodynamics (SPII) was invented to deal with problems in astrophysics involving fluid masses moving arbitrarily in 3-D in the absence of boundaries A typical example is the numerical sumulation of the fission of a rapidly lotating stal The wide variety of applications of SPH which tange fiom post-Newtonian fluid dynamics to highly supersonic metal and rok collisions show that SPH is a versatile tool There are arguments to suggest that SPII is most effective in 3-D calculations and least efficient in 1-D, but the full effectiveness of SPH is yet to be determined SPH involves the motion of a set of points Al any time the velocity and themial energy are known at these points A mass is also assigned to cach point and, for this reason, the points are referred to as particles in order to move the particles correctly during a time step it is necessary to constnuct the forces which an element of fluid would experience. These forces must be constuucted fiom the information catried by the particles [47]

The Partıcle In C'cll (PIC) method, a rombined Eulei ian-Lagrangian scheme, has been applied successfully to solve a wide variety of multifluid problens where the fluid distortions are lange It utilizes Lagıangian fluid particles to transport mass, momentum, and energy through an Fulerian mesh of cells While the use of these particles facilitates the calculation of multifluid problens, it also results in nonphysical fluctuations of the fluid quantities Furthermore, the dual mesh system of the PIC method makes great demands on computer memory capacity and calc ulation tine

In the Flund In ('cll (FLIC) nuethod, the difference equations ase very similar to those used in the PIC method Hower cr, it employs a different transport calculation which does not require the use of paiticles 'lhis reduces the memory storage requirements for a given problem and rerluces the computing time, since it is not necessary to compute the motion of the particles Furthermone, the climination of particles allows solutions which are free of the fluctuations which are characteristic of PIC [48]

\section{F 8 Monte Carlo Method}

Many problems in physics involve avelaging over many variables For example, suppose we know the position and velocity dependence of the total energy of ten interacting particles Since in 3-D cach particle has three velocity components and thee components of position, the total energy is a function of 60 variables Hence a calculation of the average energy per particle involves computing an $N=60$ dimensional integral If we divide each coordinate into $p$ intervals, there will he $p^{60}$ points to sum in this example Clearly we cannot apply the usual numerical techuiques for lange $N$, however for $N$ in the range $2-5$, the standard methods are still useful The Monte Carlo method of integration is nost suitable for the evaluation of multiple integrals for which conventional numerical methods are not generally useful

How can we use a pile of stones to measure the area of a pond? Suppose the pond is in the middle of a field of known area 1 'Throw the stones at Iandom such that they land at $\mathrm{r}$ andom within the boundary of the field and count the splashes when a stone lands in the pond The area of the pond is approximately the area of the field times the fraction of stones which make a splash This simple procedure is an example of a Monte (arlo method

More explicilly, imagine a rectangle of height $H$ and width $(b-a)$ such that $f(x)$ is within its boundaries Compute $n$ pains of landom numbers $x_{i}$ and $y_{i}$ such that $a \leq x_{i} \leq b$ and $0 \leq y_{i} \leq H$ The traction of points $x_{i}, y_{i}$ which satisfy the condition $y_{i} \leq f\left(x_{i}\right)$ is an estimate of the 1 atio of the integral of $f(x)$ to the area of the 1 cctangle Hence the estimate $F_{n}$ in the "hit on miss" method is given by

$$
F_{n}=A \frac{n_{s}}{n}
$$

where $n_{s}$ is the number of "splashes" or points below the curve, $n$ is the total of points, and 
$A$ is the area of the rectangle Note that $n$ in $(340)$ should not be confused with the number of intervals used in classical numerical methods

Another Monte Carlo procedure is based on a theosem of calculus which states that the integral is determined by the average value of the integrand $f(x)$ in the iange $a \leq x \leq b$ In or der to determine this average, we choose the $x_{i}$ at random instead of at regular intervals and sample the value of $f(x)$ For the 1-D integial, the estimate $F_{n}$ of the integral in the "sample mean" method is given by

$$
F_{n}=(b-a)\langle f\rangle=(b-a) \frac{1}{n} \sum_{i=1}^{n} f\left(x_{i}\right)
$$

where the $x_{i}$ are random numbers distributed unifonmly in the interval $a \leq x_{i} \leq b$, and $n$ is the number of trals Note that the $n$ points are chosen with 1 andom spacing in (341) For low dimensional integrals, classical methods are more accurate, but, for higher dimensional integrals (341) does better [49] 


\section{G Related Problems}

Certain physical problems have been under examination recently which are similar to our reaction-transport problem Two of these related problems are piesented here for companson

\section{G.1 The Non-linear Burgers' Equation}

Burgers' equation is a 1-D version (without pressure gradient) of the Navier-Stokes equations (85) It governs the time evolution of a $1-\mathrm{D}$ velocity field $v(x, t)$

$$
\frac{\partial v}{\partial t}+v \frac{\partial v}{\partial x}=\frac{1}{R} \frac{\partial^{2} v}{\partial x^{2}}
$$

where $R$ denotes the Reynolds number (in dimensionless units the Reynolds number $R$ is defined as $R=\nu^{-1}$ where $\nu$ denoles the kinenatic viscosity) [50]

\section{G.2 The Fisher Equation}

The Fisher equation

$$
\frac{\partial u}{\partial t}=u(1-u)+\frac{\partial^{2} u}{\partial x^{2}}
$$

where $u(x, t)$ iefers to the population clensity at a point $x$ at time $t$, was proposed by $\mathrm{R} \mathrm{A}$ Fisher in 1937 as a morel of mut ant gene propagation [51] 


\section{H Notation}

Various symbols are in use in this text Most of them are defined when they first occur in use An effort was made to tabulate them here as well, so they will all be in one place for reference Solne of the most fiequently used familiar symbols, e $g \quad e, \pi$ and $\infty$, are. intentially omitted for brevity Emphasized text appeas in atalıc style type, especially when a term is defined Variable names are in italics Units are in typewriter style

\begin{tabular}{|c|c|}
\hline $\begin{array}{l}a \\
(a q)\end{array}$ & $\begin{array}{l}1 \text { advection parameter, } 2 \text { acceleration } \\
\text { aqueous }\end{array}$ \\
\hline$A$ & $\begin{array}{l}1 \text { matrix, } 2 \text { area, } 3 \text { chemical species absurbing solar radiation } \\
4 \text { point }\end{array}$ \\
\hline$A^{*}$ & chemical species $A$ in an excited state \\
\hline$A^{T}$ & transpose of maticix $A$ \\
\hline$A^{-1}$ & inverse of matrix $A$ \\
\hline $\operatorname{det} A,|A|$ & determinant of matrix $A$ \\
\hline $\operatorname{diag} A$ & diagomal patt of matıix $A$ \\
\hline$A B$ & line segment from point $A$ to point $B$ \\
\hline$\hat{a}, \hat{b}, \hat{c}$ & vector constants \\
\hline$B$ & point \\
\hline$c$ & $\begin{array}{l}1 \text { scalar constant, } \\
2 \text { velncity of light in a vaculum, } \approx 29979 \times 10^{8} \mathrm{~m} \mathrm{~s}^{-1}\end{array}$ \\
\hline$c$ & $\begin{array}{l}1 \text { coucentration of an arbitiary gascous species, } \\
2 \text { a curve in the } x-y \text { plane, } 3 \text { heat capacity }\end{array}$ \\
\hline$c^{\prime}$ & field of complex numbers \\
\hline $\mathfrak{e}^{n}$ & $\begin{array}{l}n \text {-ilimensional complex vector space, } \\
=\left\{\left(z_{1}, z_{2}, \quad, z_{n}\right) \mid z_{1}, z_{2}, \quad, z_{n} \in \mathcal{C}\right\} \\
\text { centiglade, }=\mathrm{K}-27315\end{array}$ \\
\hline$d^{i+1}$ & Local Iruncation Euror (LTE) \\
\hline$D$ & 1 matıix, 2 diflusion parameter \\
\hline$D_{\imath}$ & chemical product of dissoriation \\
\hline$\Gamma$ & two-dimensional plane icgion \\
\hline $\mathrm{d}$ & day \\
\hline$\hat{e}^{i}$ & Total Accumulated Eror (TAE) \\
\hline e & electronic charge \\
\hline$H_{i}^{\top}$ & $i$ th equation in lineas system \\
\hline$f, F$ & 1 functions, 2 lorce \\
\hline $\mathbf{F}$ & field of scalars \\
\hline$\langle f\rangle$ & average value of $f$ \\
\hline$f_{c}$ & fluid flux due to convection \\
\hline$f_{D}$ & fluid flux due to diftusion \\
\hline$\hat{f}(\ddot{y}, t)$ & vertor function of vector variable $y$ and time variable $t$ \\
\hline$g$ & acreler ation due to gravity, $\approx 981 \mathrm{~m} \mathrm{~s}^{-2}$ at the earth's surface \\
\hline$(g)$ & gas \\
\hline$g(p)$ & scalar function of scalar variable $p$ \\
\hline$h$ & 1 time inc lement (stepsize), \\
\hline & 2 Planck's constant, $\approx 4135669 \times 10^{-15} \mathrm{eVs}$ \\
\hline$H$ & lieat flow \\
\hline $\mathrm{h}$ & hour \\
\hline$i, j, k$ & indices \\
\hline$i, \hat{j}, \hat{k}$ & orthonormal unit coordiuate vectors \\
\hline$I, I_{n}$ & $n$-dinıensional identity matrix \\
\hline$I[w]$ & functional \\
\hline
\end{tabular}




\begin{tabular}{|c|c|}
\hline $\operatorname{Im}(\lambda)$ & imaginaly component of complex $\lambda$ \\
\hline$k$ & 1 reaction rate constant, 2 thermal diffusivity, \\
\hline & 3 wave number, $=2 \pi / \lambda$ \\
\hline$k_{a}$ & photon absolption rate \\
\hline$k_{j}(x, t)$ & reaction rate coefficient functions \\
\hline$K$ & 1 thermal conductivity, 2 eddy diffusinn coefficient \\
\hline $\mathrm{km}$ & kilometes, $=10^{3} \mathrm{~m}$ \\
\hline $\mathrm{K}$ & kelvin \\
\hline$(l)$ & liquid \\
\hline$L$ & 1 matrix, 2 Lipschitz constant \\
\hline${ }_{i} \ddot{L},{ }_{i} \bar{L}$ & chemical loss rates \\
\hline L & liter \\
\hline$m$ & 1 fixcd number of iterations, 2 mass, 3 system size \\
\hline m & ineter \\
\hline$M$ & 1 matıix, 2 ratalyst \\
\hline$M$ & mole/liter \\
\hline $\min$ & minute \\
\hline mol & mole, $\approx 60221 \times 10^{23}$ \\
\hline$n$ & 1 number of iterations, '2 system size \\
\hline $\mathrm{nm}$ & nanonieter,$=10^{-9} \mathrm{~m}$ \\
\hline$N$ & $\begin{array}{l}1 \text { matix, } 2 \text { total number of chemical species involved in the } \\
\text { simulation }\end{array}$ \\
\hline$\left[\mathrm{NO}_{2}\right]$ & concentratiou of $\mathrm{NO}_{2}$, e $\mathrm{g}$ \\
\hline$O()$ & older of accuracy (big $\mathrm{O}$ ) \\
\hline$O$ & atomic oxygen \\
\hline $\mathrm{O}_{2}$ & moleculaı oxygen \\
\hline $\mathrm{O}_{3}$ & ozone \\
\hline$r$ & $\begin{array}{l}1 \text { power of } h, 2 \text { fixed point of an iudependent variable, } 3 \text { pressure, } \\
4 \text { polynomial function }\end{array}$ \\
\hline ppm & parts pej million \\
\hline$P$ & point \\
\hline${ }_{i} P$ & chemical producrion rates \\
\hline$q$ & $\begin{array}{l}\text { total number of discrete space points in } x \text {, i e number of gridpoints } \\
\text { heat }\end{array}$ \\
\hline$R$ & 1 chemical reaction rate, 2 Reynolds number \\
\hline$R_{0}$ & initial chemic al reaction rate \\
\hline${ }_{i} h(\mathbf{u}, t)$ & reactive term for ith chemical species \\
\hline$R$ & 1 field of real numbers, 2 space region \\
\hline$R^{n}$ & $n$-dimensional ieal vector space, $=\left\{\left(x_{1}, x_{2}, \quad, x_{n}\right) \mid x_{1}, x_{2}, \quad, x_{n} \in \mathcal{R}\right\}$ \\
\hline $\operatorname{Re}(\lambda)$ & real component of complex $\lambda$ \\
\hline rev & revolution \\
\hline$s$ & number of iterations \\
\hline$(s)$ & solid \\
\hline s & second \\
\hline $\mathcal{S}$ & one-dimensional plane boundary \\
\hline$t$ & independent time variable, usually in seconds \\
\hline$t_{0}$ & initial value of $t$ \\
\hline$t_{32}$ & $n$th interation of $t$ If $\Delta t$ is constant, $t_{n}=t_{0}+n \Delta t$ \\
\hline$T$ & 1 matix, 2 peiod of traveling wave, 3 temperature \\
\hline$u$ & dependent scalar vaıiable \\
\hline$u^{n}$ & $n$th time iteration of $u$ \\
\hline$u_{j}^{n}$ & $j$ th spatial iteration and $n$th temporal iteration of $u$ \\
\hline$u^{n+1, s+1}$ & $s+1$ conector iteration of $n+1$ predictor iteration of $u$ \\
\hline
\end{tabular}




\begin{tabular}{|c|c|c|}
\hline$\dot{u}=\left(u_{1}\right.$ &,$\left.u_{q}\right)^{T}$ & column vector of $q$ components, usually of $q$ spatial points \\
\hline & & partial derivative of $u$ with respect to $x$ \\
\hline$u_{x y}$ & & second partial derivative of $u$ \\
\hline$i u$ & & concentration of $i$ th chemical species \\
\hline$i u_{j}^{n}$ & & $\begin{array}{l}j \text { th spatial itesation and } n \text {th temporal iteration of concentsation of } \\
i \text { th chemical species }\end{array}$ \\
\hline $\mathbf{u}=\left({ }_{1} u\right.$ &,$\left.N^{\prime} u\right)^{I}$ & $\begin{array}{l}\text { column vector of } N \text { components, usually of concentiations of } N \\
\text { chemical constituents }\end{array}$ \\
\hline (I & & matrix \\
\hline$v$ & & phase velocity of traveling wave, $=\lambda / T=\omega / k$ \\
\hline$I^{r}$ & & volıme \\
\hline$I^{r}$ & & velocity of a fluid \\
\hline $\mathrm{V}$ & & vector space \\
\hline $\mathrm{V}$ & & volt \\
\hline$x, y, z$ & & independent space variables \\
\hline$x, y$ & & dependent vector variables \\
\hline$x^{0}$ & & initial value of $\hat{x}$ \\
\hline$\dot{x}_{i}^{j}$ & & $i$ th component of $j$ th iteration of vector $\hat{x}$ \\
\hline$\hat{x}^{i}:+1, \gamma$ & & Jacobi conection, computed as iteration $k+1$ of Jacobi method \\
\hline$\left\{\hat{x}^{k}\right\}_{i=0}^{\infty}$ & & infinite scquence of vectors $\hat{x}^{k}$ \\
\hline$\dot{x}, \ddot{x}$ & & first, second, etc derivative of $x(t)$ with iespert to $t$ \\
\hline$y^{\prime}, y^{\prime \prime}$ & & first, second, etc delivative of $y$ \\
\hline$j_{y}$ & & $j$ th dependent variable $y$ in a system of ODEs \\
\hline$y_{m}$ & & amplitude of traveling wave \\
\hline$y^{(n)}$ & & nth deivative of $y$ \\
\hline$\dot{y}\left(t_{i}\right)$ & & artual value of $y(t)$ evaluated at $t_{i}$ \\
\hline$\hat{y}^{i}$ & & munerical approximation to $y\left(t_{i}\right)$ \\
\hline$a$ & & diffusion coeflicient \\
\hline$\Gamma^{\prime}$ & & a culve in the $x-y$ plane \\
\hline$\delta_{i j}$ & & Kuonecker delta, $=1$ if $i=j, 0$ if $i \neq j$ \\
\hline$\Delta t$ & & time increment (may be constant, may be variable) $\Delta t=t_{n+1}-t_{n}$ \\
\hline$\Delta x$ & & spare increment \\
\hline$\triangle\left[\mathrm{NO}_{2}\right]$ & & change in concentration of $\mathrm{NO}_{2}, \mathrm{eg}$ \\
\hline$\nabla$ & & differential vertor operator, in $3-\mathrm{D}=\frac{\partial}{\partial x} \dot{i}+\frac{\partial}{\partial y} \dot{i}+\frac{\partial}{\partial z} \dot{k}$ \\
\hline$\epsilon$ & & toleiance \\
\hline$\hat{\eta}$ & & initial condition vertor \\
\hline$\theta_{1}, \theta_{2}$ & & direction angles of outward nosmal to plane segion boundasy \\
\hline$\kappa_{t}$ & & arbitıary constants in ODE solution \\
\hline$\lambda$ & & $\begin{array}{l}1 \text { wavelength of solar radiation photon, } 2 \text { wavelength of traveling } \\
\text { wave, } 3 \text { eigenvalue }\end{array}$ \\
\hline$\mu$ & & combined iteration constant \\
\hline$\nu$ & & 1 fiequency of solas radiation photon, 2 kinennatic viscosity \\
\hline$\xi$ & & point in interval of interest where Taylon remainder is a single tem \\
\hline$\rho$ & & 1 advection coefficient, 2 density \\
\hline$\rho(4)$ & & spectral radius of inatiix $A$ \\
\hline$\sigma$ & & specific heat \\
\hline$\phi$ & & 1 quantum yield, 2 atmospheric eddy flux \\
\hline$\grave{\phi}$ & & approxinnating function \\
\hline$\phi(\ddot{y}, t)$ & & vector function of vector variable $y$ and tirne variable $t$ \\
\hline$\dot{\psi}$ & & increment function \\
\hline$\dot{y}(x)$ & & palficular integral in ODE solution \\
\hline$\omega$ & & 1 relaxation weighting factor, 2 angular liequency, $=2 \pi / T$ \\
\hline
\end{tabular}


legion in $\mathcal{R}^{n}$ 


\section{Abbreviations}

Various abbreviations are in use in this text As was noted previously for the notation, most of the abbleviations ale defined when they first occur in use, but are tabulated here as well

\begin{tabular}{|c|c|}
\hline$B C^{1}$ & Boundary Condition \\
\hline BDF & Backward-Difference Foımula \\
\hline $\mathrm{BE}$ & Backward Euler \\
\hline $\mathrm{BVP}$ & Boundary Value Problem \\
\hline C'HEMSODE & a stiff ODE solver for the equations of chemical kinetics \\
\hline C:PU & Central Processing Unit \\
\hline $\mathrm{DF}$ & Differcutial Equalion \\
\hline DEC & Digital Equipment Corporation \\
\hline DHM & Damped Hatmonic Motion \\
\hline FDM & Finite Difference Method \\
\hline $\mathrm{FE}$ & For ward Euler \\
\hline FEM & Finite Element Method \\
\hline FLIC & Fluid In C'ell \\
\hline ETCS & Finite Time Central Space \\
\hline GS & Grauss-Seidel \\
\hline IBVP & Initial Boundary Value Problem \\
\hline IC & Initial ('ondition \\
\hline IN & Interconnection Network \\
\hline IVP & Initial Value Problem \\
\hline I.LNI, & Lawrence Livermore National Laboratory \\
\hline LMM & Linear Multistep Method \\
\hline LSODE & Livermore Solver for Ordinary Differential Equations \\
\hline LTE & Local Truncation Enior \\
\hline LW & Lax-Wendioff \\
\hline MIMD & Multi-Instunction Multi-Data \\
\hline MPI & Message-Passing Interface \\
\hline NR & Newton-Raphsou \\
\hline NUMOL & Numerical Method of Lines \\
\hline ODE & (odinary Differential Fquation \\
\hline OS & Operating System \\
\hline $\mathrm{PC}$ & Predictor-Cor rector \\
\hline PD & Positive Definite \\
\hline PDE & Partial Diflerential Equation \\
\hline PIC & Particle In Cell \\
\hline PTD & Preconditioned Time Differenring \\
\hline RISC & Reduced Instı uction Set Computer \\
\hline RK & Runge-Kutta \\
\hline $\mathrm{RP}$ & Relative Peiformance \\
\hline SDD & St.1ictly Diagonally Iominant. \\
\hline SHM & Simple Harmonic Motion \\
\hline SIMD & Single-Instruction Multi-Data \\
\hline SOR & Successive Over-Relaxation \\
\hline $\mathrm{SPH}$ & Sinoothed Particle Hydıodynamics \\
\hline TAE & Total Accumulated Friol \\
\hline UW & Upwind Differ encing \\
\hline
\end{tabular}




\section{References}

[1] Wanneck, Peter Chemistry of the Natural Atmosphere San Diego, CA Academic Piess, $1988, x i, 60,100$

[2] Graedel, T E, Donald T Hawkins, and Lany D ('laxton Atmosphenc Cihemical Compounds Sources, Occurrence, and Bioassay Oılando, FL Academic Press, 1986, $2-10$

[3] Finlayson-Pitts, Barbara J and James N Pitts, J I Atmospheric Chemistry Fundamentals and Fxperimental Techniques New Yolk, NY Wiley, 1986, 8,60

[4] Wayne, Richard P ('hemestry of the. Atmospheres An Introduction to the Chemestry of the Atmospheres of Farth, the Planets, and therr Satcllates Oxfurd Clarendon Piess, 1985, 106-107

[5] Miller, $J$ A, R J Kee, and C K Westbrook "Chemical kinetics and combustion modeling", Annu Rev Phys Chem, 41 (1990), 345-387

[6] Meyer, P D , A J Valecchi, S F Ashby, and P E Saylor "A numerical investigation of the conjugate gradient method as applied to three-dimensional gioundwater flow problems in randomly heterogencous porous media", Water Resources Res, 25 (1989), 1440-1446

[7] Wucbbles, D J, K F' Giant, P S Connell, and J E Pemes "The inle of atmospheric chemistry in climate (hange", APC'A Journal, 39 (1989), 1

[8] Kinnison, D F, K F (riant, P S Connell, D A Rotman D J Wueblles, "The chenic al and racliative efferts of the Mount Pinatubo cruplion", J Geophys Res, 99 (1994)

[9] Teranoto, 'I ed Deep Orean C'inculation Physical and C'hemical Aspects New York, NY Elsevier, 1993

[10] Aro, C J "CHEMSODF A stiff (ODE solver for the equations of chemical kinetics", Comput Phys Commun, 97 (1996), 304-314

[11] McQuarie, Donald $\Lambda$ and Peles A Rork Gencral Chemestry, 3rd ed New York, NY W H Freeman and Co, 1991, 548-557

[12] Rotman, I) A, D J Wuebbles, and J E Penner "Atmospheric chemistry using massively parallel computess" in AMS fifth annual symposium on global change studies (American Mathematical Society, Providence, RI, 1994)

[13] Dabdub, D and J H Seinfeld "Extrapolation techniques used in the solution of stiff ODEs associated with chemical kinctics of air quality nodels", Atmos Envnron, 29 (1995), 3

[14] Lambert, J D Numencal Methods for Ordmary Dufferential Systems New Yok, NY Wiley, 1992, 2,4-6,11-12,27,45,56-57,107,149,213-220

[15] Byne, ( $\rightarrow$ D and Alan (' Hindmarsh "Stiff ODE Solvels A Review of ('unent and Coming Attiactions", J Comput Phys, 70 (1987), 1

[16] Schiesser, W E The Numeracal Method of Lines Integration of Partial Differental Fiquatıons San Diego, ('A Academic Press, 1991, 1-10

[17] Robeison, John A and Clayton T Crowe Engmeenng Flund Mechanus, 3rd ed Boston, MA Houghton Miffin, 1985, 112,164-165 
[18] Rodigue, G and D Wolitzer "Preconditioned time differencing for the parallel solution of the heat equation", Proceedings of the Fourth SIAM Conference on Parallel Processing for Scientific Computing, (1989), 268-270

[19] Street, Robert L Analysıs and Solutıon of Partial Differential Equatıons Monterey, CA Brooks/Cole, 1973, 1-3,12-16,33,38-44,53-56,253-254,281-285

[20] Ortega, James M and William (: Poole, Ji An Introduction to Numerical Methods for Differentzal Equations Masshfield, MA Pitman, 1981, 243,245,248

[21] Rodıiguc, G and D Wolitzer "Expanding Stability Regions of Explicit AdvectiveDiffusive Finite Difference Methods by Jacobi Preconditioning", Numercal Methods for Partial Differentzul Equatıons, 11 (1995), 339-353

[22] Aın, $\mathrm{C} J$ and $(\mathrm{x} \mathrm{H}$ Rodrigue "Preconditioned time differencing for stifl ODEs in diunual atmospheric kinetics", Comput Phys Commun, 92 (1995), 27-54

[23] Aro, C J "A stiff ODE preconditiones based on Newton Linearization", App Num Math , 21 (1996), 335-352

[24] Stott, P A and R S Harwood "An implicit time-stepping scheme for chemical species in a global atmospheric (irculation model", Ann Genphysicae, 11 (1993), $377-388$

[25] Verwer, J ( $\mathrm{x}$ and D Simpson "Explicit methods for OD)Es from atmospheric chemistry", App Num Math, 18 (1995), 413-430

[26] Steinfeld, J I, J S Francisco, and W L Hase Chemucal Kinetıcs and Dynamics, Englewood Cliffs, NJ Prentice Hall, 1989

[27] Aır, C J , Al Fianz and Dale Slone "A Highly Stable Explicit Technique for Stiff Reaction-Tiansport PDEs", 8th SIAM Conference on Purallel Processing for Sirentific C'omputing, Philadelphia, PA SIAM, 1997

[28] Resnick, Robert and David Halliday Physics, 3rd ed Ncw Yolk, NY Wiley, 1977, $121-122,301-302,322,371,404-410,457-460,477,480-481$

[29] Kreyszig, Firwin Advansed Fngineering Mathematics, 3rd cd New York, NY Wiley, $1972,12,18,62-68,78,273-274,415-416,130-433,675-677$

[30] Tipler, Paul A Physıcs for Screntısts and Engineers, 3rd ed, Extended Version New York, NY Wolth, 1991, 486,517

[31] Schlichting, H Boundary Layer Theory New York, NY McGiaw-Iill, 1979

[32] Mitchell, A R and D F Giffiths The Finite Difference Method in Partaal Differential Equatrons Chichester Wiley, 1980, 244-245

[33] Itô, Scizô Translations of Mathematical Monographs, vol 114 Diffusion Equatzons Providence, RI Amer Math Soc, 1979, 1-3

[34] Mészáıos, E Atmospherıc C'hemıstry Fundamental Asperts Amsterdam Elsevier, $1981,47-48$

[35] Protter, Murray H and Charles B Money, Jr C'ollege Calculus wath Analytic Greometry, 2nd ed Reading, MA Addison-Wesley, 1970, 544,658-668

[36] Beauregard, Raymond A and John B Fraleigh A Furst C'ourse on Linear Algebra With Optional Introdurtuon to Groups, Rings and Fields Atlanta, (iA Houghton Mifflin, 1973, 2,65-66,160-162,265-266,374 
[37] Burden, Richard L and J Douglas Faires Numertral Analysis, 5th ed Boston, MA Prindle, Weber and Schmidt, 1993, 26,46-50,56-57,239,248,271,314,321,325-326,347$349,353,358,371,373,379,4() 6-416,657-659$

[38] Nering, Evar D Linear Algebra and Malizx l'heory, 2nd ed New York, NX Wiley, 1970,104

[39] Simmons, George F Differential Fquations with Applications and Hislorical Notes New York, NY McGiaw-Hill, 1972, 1-4,47-48,124-125

[40] Smith, G D Numerical Soluton of Partial Differentzal Equations Finite Difference Methods, 3rd ed Oxford (larendon Press, 1985, 1,6-8,175-176

[41] Hildebrand, F B Introductuon to Numerical Analysis, 2nd ed New York, NY McGraw-Hill, 1974, 1-3

[42] Conte, S D and Canl de Boor Elementary Numencal Analysus An Algorithmic Approach, 3rd el New Yolk, NY McGraw-Hill, 1980, xi

[43] Hishfelder, J O "Applicd Mathematics as used in Theoretical Cliemistry", Amertcan Mathematical Society Symposium (1963), 367-376

[44] Radhakrishnan, Krishnan and Alan (" Hindnarsch Descriptıon and Use of LSODF, the Livermore Solver for Ordmary Differental Equations Repoit UCRL-ID-113855, Lawrence Livermore Laboratory, Livenmore, CA, 1993, 33

[45] Zima, Ilans and Batbara Chapman Supercomplers for Purallel and V'ector Computers New York, NY Addison-W'sley, 1991, 18

[46] Lewis, Ted G and Itesham El-Rewini with In-Kyu Kim Introduction to Parallel Computıng Englewood Clifls, NJ Pientice Hall, 1992

[47] Monaghan, J J "An Intinduction to SPIL", Comput Phys Commun, 48 (1988), $89-96$

[48] Gentıy, Richard A, Rohert E Maılin, and Bart J Daly, "An Eulerian Diflerencing Method for Unsteady Compressible. Flow Pioblems", I Comput Phys, 1 (1966), $87-118$

[49] (rould, Harvey and Jan Tobochnik, An In/roduction to Computer Sumulation Methods Applacations to Physiral Systems, Part 2 Reading, MA Addison-Wesley, 1988, 326-331

[50] Breuer, Heinz-Peter and Francesco Petruccione "On the Numerical Integration of Burgers' Equation by Stochastic Simulation Methods", Computer Physics Communications, 77 (1993), 207-218

[51] Parekh, N and Sanjay Puri "A New Numerical Scheme for the Fisher Equation", $J$ Phys A Math Gen, 23 (1990), L1085-L1091 


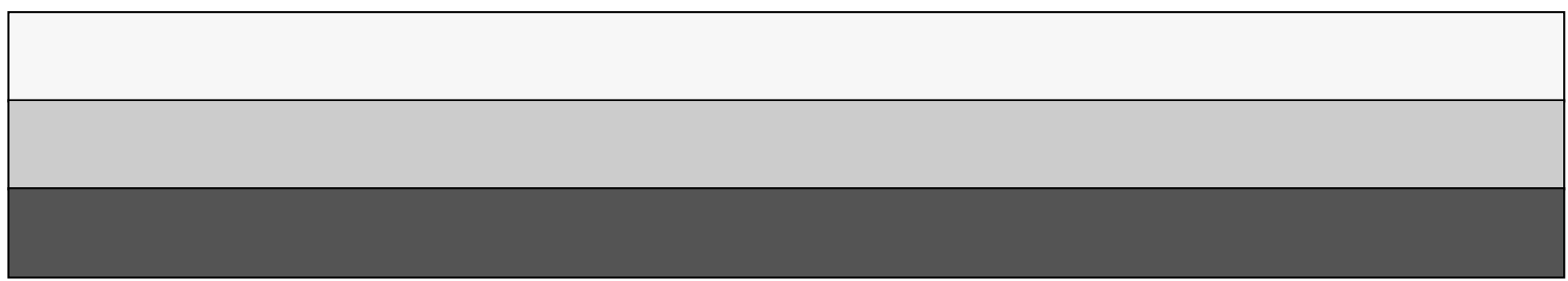

